\title{
Index to the Distributions of Mathematical Statistics
}

\author{
Frank A. Haight*
}

(February 15, 1960)

\begin{abstract}
A fairly complete index of references to results on statistical distributions published before January 1958 is presented. The material given for each distribution is a list of references relating to: (a) functions and constants which characterize the distribution, $(b)$ derived distributions, $(c)$ estimation, $(d)$ testing statistical hypotheses, $(e)$ miscellaneous. The distributions covered are characterized as normal, type III, binomial, discrete, distributions over $(a, b)$, distributions over $(a, \infty)$, distributions over $(-\infty, \infty)$, miscellaneous univariate, miscellaneous bivariate, and miscellaneous multivariate. The number of entries varies from one or two for less well-known distributions to several hundred for the normal distribution. This index should serve to eliminate unnecessary derivation of results already in the literature.
\end{abstract}

\section{Introduction}

\section{Background}

The author began this index in April 1954 with the limited intention of supplying his students at Auckland University College, New Zealand, with a small reference pamphlet. It appeared that no textbook of mathematical statistics contained a complete treatment of all the distributions that a student might encounter. Thus an index was needed to facilitate selection of the appropriate book. In 1955 the collected results were mimeographed, and during the next three years several hundred copies of this early version were sent out in response to requests.

Then, at the invitation of the National Bureau of Standards, the author spent the summer of 1958 at the Bureau's Statistical Engineering Laboratory supplementing and editing the index for publication in the present form.

In the course of this work, a number of additional sources have been included and the journal coverage has been extended through 1957. Information supplied by readers of the original version has been incorporated, and various mistakes have been corrected.

\section{Organization}

The material given under each distribution consists of a number of entries, most of which are provided with one or more references. In the case of the normal distribution with mean $\mathrm{m}$ and variance $\mathrm{v}$ (No. 1.1) the number of entries is fairly large, and therefore the standard order is most easily seen:

$A$. Functions and parameters

$B$. Derived distributions

(a) of linear quantities

(b) of quadratic quantities

*Present address: Institute of Transportation and Traffic Engineering, University of California, Los Angeles, Calif.
C. Estimation
(a) point

(b) interval

D. Testing statistical hypotheses

(a) by linear statistics

(b) by quadratic statistics

E. Miscellaneous

These categories are by no means always used for less important distributions. With the limited information available, a complete listing of the headings in such cases would be wasteful as the majority would be empty. Keeping in mind the above order, it should not be difficult to find the required entry.

Occasionally an entry will be indented; such an entry should be read as a continuation of the preceding one.

\section{References}

The references to the literature are of the following types:

A. Coded.

(a) Journals, e.g., $[c] 4: 17$, which refers to page 17 of the 4th volume of the journal designated as [c] in table 1.

(b) Books, e.g., [12]53, which refers to page 53 of the book designated as [12] in table 2.

B. Uncoded, e.g., Trans. Am. Math. Soc. 17:382, conforming to the usual volume and page reference style.

$C$. Reviews

(a) Mathematical Reviews is designated by MR,

(b) Zentralblatt für Mathematik is designated by Z.

$\mathrm{MR}$ and $\mathrm{Z}$ references will in no case offer a review of a paper appearing in coded journals and therefore may be considered to indicate publications in obscure (from the point of view of the present work) sources. Moreover, every effort has been made to avoid MR or $\mathrm{Z}$ references to an uncoded paper quoted and very few duplications of this sort should be found. 
The choice between direct (i.e., coded or uncoded) and indirect (i.e., review) references is frequently available. The one that was actually inspected is given. All direct references were collected before the search of MR and Z. Consequently, each entry corresponding to a direct reference is based on the paper, and never its review, and each entry corresponding to an indirect reference is based on the review and never the paper.

As it is difficult to distinguish priority in a large number of references, a chronological table of the coded and review references is provided. This table also shows what volumes have been systematically searched in the preparation of this index.

\section{Criteria for Inclusion}

A. Distributions. As a general principle, a distribution is included if its density (or probability) function is a known, explicit function. The following exceptions may be noted:

(a) Certain families of distributions are mentioned, e.g., Pearson and Koopman, whose densities are specified only implicitly.

(b) Certain distributions are mentioned in terms of their cumulative probability function or characteristic function.

$B$. Entries. The general principle governing the selection of entries is that each entry must exhibit a property of the distribution in question. Exceptions to this rule generally take one of the following forms :

(a) Historical information about well-known distributions, although not systematically sought, may in some circumstances be included.

(b) Important applications, such as those which led to the discovery of the distribution, are usually supplied.

(c) Bibliographies.

Reference to tables has been excluded in almost every case.

It is clear that applications must be severely limited. With a slight exaggeration, several whole branches of statistics may be considered applications of some particular distribution, as exhibited for example in the following table:

\section{Distribution}

Binomial
Normal
Lognormal
Poisson
Deterministic

Application

Quality control
Analysis of variance
Probit analysis
Random processes
Applied mathematics

\section{Relationship Between Distributions}

In some cases (such as 2.1 and 2.3) the relationship between two distributions is asserted in their designation. In others (such as 5.3 and 5.15) a very close connection is not pointed out. In most cases, however, known relationships are simply listed among the miscellaneous properties of both distri- butions. In choosing between these procedures, an attempt has been made to follow current statistical usage and terminology.

Very similar principles have been used to decide for or against independent listing. If one distribution is relatively important and its equivalent much less so (for example Chi-square and Erlang), they are listed together. In other cases independent reputation seems to justify independent categories.

It must certainly be supposed that many of the trivial distributions of section 8 could be included in some larger category or even combined with each other. Such a systematic classification which would exhibit all connections, even if worth doing, is certainly removed from the purpose of this index, and has hardly been attempted.

For example, it is well known that no. 8.1 contains as special cases all the distributions of sections 1 and 2 ; very likely it also contains dozens of others listed. Nevertheless, to indicate this situation by a system of subheadings, applied to all entries, would quickly undermine the utility of the whole work, since it is the special case, rather than the general principle, that occurs in statistical practice.

\section{Notation and Terminology}

In univariate distributions the stochastic variable is always denoted by $\mathrm{x}$, in bivariate by $\mathrm{x}$ and $\mathrm{y}$ and in multivariate by $x_{1}, \ldots, x_{k}$, quite regardless of the domain of definition. This usage departs from that of certain authors in two respects:

(a) The letter $n$ is not used for a discrete variable.

(b) The statistic obeyng a particular distribution is not used in the density. For example in Student's " $t$ " distribution, we write

$$
\left(1+\frac{x^{2}}{\nu}\right)^{-\frac{1}{2}(\nu+1)}
$$

rather than

$$
\left(1+\frac{t^{2}}{\nu}\right)^{-\frac{1}{2}(\nu+1)}
$$

This practice is justified not only by the need for uniformity but by the belief that the alternative is wasteful of the alphabet: $t, F, z, D, \ldots$ Similarly we prefer to call distributions by the names of their discoverers (or reputed discoverers) rather than by the symbol used to denote some statistic found to satisfy them. Of course, all known designations are found in the final index.

In many books the expression $\mathrm{f}(\mathrm{x})$ is employed to denote a probability density. However $\mathrm{f}$ is commonly used in mathematics for an arbitrary function. $\mathrm{D}(\mathrm{x})$ has therefore been selected as a more distinctive means of representing this special function.

In the discrete case $\mathrm{D}(\mathrm{x})$ replaces the probability distribution, which is often written $\mathrm{p}_{n} . \mathrm{C}(\mathrm{x})$ is the cumulative function.

When we come to the characteristic function the situation is a little more complicated. Using $\mathrm{t}$ for 
the variable, statistical works generally have to define several symbols for characteristic functions of various quantities, for example:

$\chi(t)=$ characteristic function of distribution of $x$ $\phi(t)=$ characteristic function of distribution of $\mathrm{n} \overline{\mathrm{x}}$

$\xi(t)=$ characteristic function of distribution of $\overline{\mathrm{x}}$, etc.

As we will be dealing with many different statistics and possibly their characteristic functions, it is more economical and systematic simply to abbreviate by the following system: $\mathrm{Ch}(\mathrm{x}), \mathrm{Ch}(\mathrm{n} \overline{\mathrm{x}}), \mathrm{Ch}(\overline{\mathrm{x}})$, etc. Thus it is not necessary to select a new letter to denote the characteristic function of each new statistic.

However, this practice leads to equations like

$$
\mathrm{Ch}(\mathrm{x})=\mathrm{e}^{-\frac{1}{2} \mathrm{vt}}
$$

which may be offensive to some, however clear the meaning. Such readers are advised to interpret the equality sign as an abbreviation for the verb "is."

This interpretation has another important connection with the notation being used. A variety of verbs has been employed to describe the relation between a stochastic variable and its distribution, for example:

$\mathrm{x}$ obeys the normal distribution with mean $\mathrm{m}$ and variance $\mathrm{v}$,

$\mathrm{x}$ follows the normal distribution with mean $\mathrm{m}$ and variance $\mathrm{v}$,

$\mathrm{x} i$ a normal variable with mean $\mathrm{m}$ and variance $\mathrm{v}$.

It seems equally felicitous to assert this relationship by the convenient abbreviation

$$
\mathrm{D}(\mathrm{x})=\mathrm{N}(\mathrm{m}, \mathrm{v}),
$$

which may, if preferred, be regarded not as a mathematical equation but as shorthand. In any case it makes possible an unambiguous condensation of the facts.

Similar remarks apply to the expressions $\mathrm{MGF}(\mathrm{x})$, $\mathrm{FD}(\mathrm{p})$ which are used to mean moment generating function of the distribution of $\mathrm{x}$ and fiducial dis. tribution of the parameter $p$.

Another application of this use of the equality sign relates to the symbols C.- R.(p), $\operatorname{MLE}(p), \operatorname{MME}(p)$, $\operatorname{UMVUE}(p), \operatorname{BANE}(p)$, and is exemplified by the following:

$$
\begin{gathered}
\text { C. }-\mathrm{R} \cdot(\sigma)=\mathrm{v} / 2 \mathrm{n} \\
\operatorname{MLE}(v)=\mathrm{s}^{2} \\
\operatorname{UMVUE}(v)=\mathrm{ns}^{2} /(\mathrm{n}-1) .
\end{gathered}
$$

For the meanings of these and other abbreviations, the reader is referred to the following list:

$$
\begin{gathered}
\mathrm{D}(\mathrm{x}) \text { - Density or probability function of a } \\
\text { stochastic variable } \mathrm{x}
\end{gathered}
$$

$\mathrm{C}(\mathrm{x})$ - Cumulative distribution function of $\mathrm{x}$

$\mathrm{Ch}(\mathrm{x})$ - Characteristic function of distribution of $\mathrm{x}$

MGF( $\mathrm{x}$ - Moment generating function of distribution of $\mathrm{x}$

PGF $(x)$ - Probability generating function of $x$

$\mathrm{FD}(\mathrm{p})$-Fiducial distribution of a parameter $\mathrm{p}$

$\mathrm{m}$-Mean of a population

$\bar{x}$-Mean of a sample

$\mathrm{V}=\sigma^{2}-$ Variance of a population

$\mathrm{s}^{2}$-Variance of a sample

$$
\text { (i.e., } \left.\mathrm{s}^{2}=\sum_{i=1}^{\mathrm{n}}\left(\mathrm{x}_{\mathrm{i}}-\overline{\mathrm{x}}\right)^{2} / \mathrm{n}\right)
$$

$\mu_{\mathrm{k}}$ - kth central moment of a population

$\alpha_{\mathrm{k}}$ - kth moment about the origin for a population

$\mathrm{K}_{\mathrm{k}}$ - kth cumulant

$\mathrm{r}$-Correlation coefficient in a sample

$\rho$-Correlation coefficient in a population

$\xi$-Median

GM-Geometric mean

HM-Harmonic mean

$\mathrm{n}$-Number of items in a sample

$\beta$ - Slope of regression line in a population

b-Slope of regression line in a sample

$\sim$-Asymptotic (=large sample)

$\beta_{1}, \beta_{2}$-Pearson's betas

C.-R.(p)-Cramér-Rao lower bound for variances of estimates of the parameter $\mathrm{p}$

MLE(p)-Maximum likelihood estimate of the parameter $\mathrm{p}$

MME(p)-Minimax estimate of the parameter $p$

$M \chi^{2} \mathrm{E}(\mathrm{p})$-Minimum Chi-square estimate of the parameter $\mathrm{p}$

UMVUE(p)-Uniformly minimum variance unbiased estimate of the parameter $p$

BANE(p) - Best asymptotically normal estimate of the parameter $\mathrm{p}$

LR-Likelihood ratio

L-The likelihood function $\Pi \mathrm{D}\left(\mathrm{x}_{\mathrm{j}}\right)$

Seq-Sequential

OC-Operating characteristic 
BCR - Best critical region

Q-A quadratic form

E-Expectation

The author wishes to thank Churchill Eisenhart for arranging his visit to the National Bureau of Standards, and Lola S. Deming for helpful advice on the typescript and for invaluable assistance in readying final copy for printed publication. Also, he is grateful to Dean L. M. K. Boelter (acting on behalf of the Regents of the University of California) for granting two-months' leave from the Institute of Transportation and Traffic Engineering.

Finally, it is too much to hope that a work of this character and magnitude is entirely free from error. The author will welcome notification of necessary corrections, and of important omissions.

\section{Distributions}

\section{Normal}

\subsection{Normal $(\mathrm{m}, \mathrm{v})$}

\section{A. Functions and Parameters}

$\mathrm{D}(\mathrm{x})=(1 / \sqrt{2 \pi \mathrm{v}}) \mathrm{e}^{-(\mathrm{x}-\mathrm{m})^{2} / 2 \mathrm{v}}: \quad[6] 108, \quad[5] 34, \quad[4] 5 \%$, $[8] 91,[9] 243$.

$\mathrm{Ch}(\mathrm{x})=\exp \left(-\frac{1}{2} \mathrm{vt}^{2}+\mathrm{mit}\right): \quad$ [1]211, [5]62.

$\operatorname{MGF}(\mathrm{x})=\exp \left(\frac{1}{2} \mathrm{vt}^{2}+\mathrm{tm}\right): \quad[6] 112$.

Derivatives etc. [d]2:181.

Transformations: $[c] 39: 290$.

Obtained from Pearson's differential equation: $[4]^{72}$.

Called Type VII: $\quad[11] 45$.

Limit of binomial: [4]58.

Variance of $\overline{\mathrm{x}}$ and $\mathrm{s}: \quad[3] 42$.

$\operatorname{Var}\left(\mathrm{m}_{3}\right)=6 \mathrm{v}^{3} \mathrm{n}^{-1}, \operatorname{var}\left(\mathrm{m}_{4}\right)=96 \mathrm{v}^{4} \mathrm{n}^{-1}$ and many other constants: [2]224.

Calculation of constants and numerical examples: [11]88.

Mean deviation $\quad \mathrm{E}|\mathrm{x}-\mathrm{m}|=(2 \mathrm{v} / \pi)^{\frac{1}{2}}=.79788 \sigma$ : [1]258.

Probable error $=.6745 \sigma: \quad$ [4]58.

$\alpha_{2 \mathrm{k}}=(2 \mathrm{k}-1) \mathrm{v}^{\mathrm{k}}: \quad[5] X I I,[8] 98$.

Quasi-range: $\quad[d] 24: 603$.

\section{B. Derived Distributions}

$\mathrm{D}(\overline{\mathrm{x}})=\mathrm{N}\left(\mathrm{m}, \mathrm{vn}^{-1}\right): \quad[9] 2 \% 0,[6] 10.2,[2] 243,[4] 100$ $[w] 1: 93$.

$\mathrm{D}(\overline{\mathrm{x}} / \mathrm{s}): \quad[3] 139$.

$\mathrm{D}\left[(\mathrm{n}-1)^{\frac{1}{3}} \mathrm{~s}^{-1}(\overline{\mathrm{x}}-\mathrm{m})\right]=$ Student $(\mathrm{n}-1): \quad[6] 217,[5] 98$, [2]239, [4]112, [w]1:74.
$\mathrm{D}\left[\mathrm{s}^{-1}(\mathrm{n}-1)^{\frac{1}{2}} \overline{\mathrm{x}}\right]$, where $\mathrm{x}_{1}$ are samples from $\mathrm{N}\left(\mathrm{m}_{1}, \sigma\right)$; $\mathrm{m}_{1}$ not all equal: $[d] 19: 406$.

$\mathrm{D}[(\overline{\mathrm{x}}-\mathrm{m}) /$ range $]: \quad[d] 22: 469$.

D (range) etc.: MR13:762.

$\mathrm{D}\left(\Sigma \mathrm{k}_{1} \mathrm{x}_{\mathrm{i}}\right)=\mathrm{N}\left(\Sigma \mathrm{k}_{1} \mathrm{~m}_{\mathrm{i}}, \Sigma \mathrm{k}_{\mathrm{i}}^{2} \mathrm{v}_{\mathrm{i}}\right): \quad$ [4]99, [8]92.

$[(\mathrm{x}-\mathrm{m} / \sigma)]^{2}$ is Chi-square, $(\mathrm{x} / \sigma)^{2}$ is noncentral Chisquare, $(x-m)^{2}$ is Type III, product of normal variables is Bessel, quotient normal for large $\mathrm{m} / \sigma: \quad[18] 1-150$.

$\mathrm{D}\left[\frac{\mathrm{m}_{1}-\mathrm{m}_{2}\left(\mathrm{x}_{1} / \mathrm{x}_{2}\right)}{\sqrt{\mathrm{v}_{1}+\mathrm{v}_{2}\left(\mathrm{x}_{1}^{2} / \mathrm{x}_{2}^{2}\right)}}\right]=\mathrm{N}(0,1), \quad \mathrm{m}_{2} \gg \sigma_{2}$ :

[2]253.

$\mathrm{D}\left(\overline{\mathrm{x}}_{1}-\overline{\mathrm{x}}_{2}\right)=\mathrm{N}\left(\mathrm{m}_{1}-\mathrm{m}_{2}, \mathrm{v}_{1} / \mathrm{n}_{1}+\mathrm{v}_{2} / \mathrm{n}_{2}\right)$ :

[4]100.

$\mathrm{D}\left[\frac{\overline{\mathrm{x}}_{1}-\overline{\mathrm{x}}_{2}}{\mathrm{v}\left(\frac{1}{\mathrm{n}_{1}}+\frac{1}{\mathrm{n}_{2}}\right)}\right]=\mathrm{N}(0,1)$ when $\mathrm{m}_{1}=\mathrm{m}_{2}$ and $\mathrm{v}_{1}=\mathrm{v}_{2}=\mathrm{v}$ :

[6]263.

$\mathrm{D}\left[\frac{\left(\overline{\mathrm{x}}_{1}-\overline{\mathrm{x}}_{2}\right)-\left(\mathrm{m}_{1}-\mathrm{m}_{2}\right)}{\left(\mathrm{n}_{1} \mathrm{~s}_{1}^{2}+\mathrm{n}_{2} \mathrm{~s}_{2}^{2}\right)^{\frac{1}{2}}}\left(\frac{\mathrm{n}_{1}+\mathrm{n}_{2}-2}{\mathrm{n}_{1}^{-1}+\mathrm{n}_{2}^{-1}}\right)^{\frac{1}{2}}\right]$

Student $\left(\mathrm{n}_{1}+\mathrm{n}_{2}-2\right):$ [4]112, [3]109, 112, [5].98, [c]29:350, [c]33:252, MR8:42.

$\mathrm{D}\left[\left(\frac{\mathrm{n}_{1}+\mathrm{n}_{2}-2}{2}\right)\left(\frac{\mathrm{n}_{1}\left(\overline{\mathrm{x}}_{1}-\mathrm{m}_{1}\right)^{2}+\mathrm{n}_{2}\left(\overline{\mathrm{x}}_{2}-\mathrm{m}_{2}\right)^{2}}{\mathrm{n}_{1} \mathrm{~s}_{1}^{2}+\mathrm{n}_{2} \mathrm{~s}_{2}^{2}}\right)\right]$

$=$ Snedecor $(2, \mathbf{n}-2)$, confidence ellipse:

[4]132.

$\mathrm{D}\left(\mathrm{k}^{\text {th }}\right.$ value from top $): \quad[1] 3 \% 4,[d] 25: 565$.

$\mathrm{D}($ smallest sample value $)=$ No. 8.40: $[\mathrm{g}]$ 42:408.

$\mathrm{D}\left[\frac{1}{2} \mathrm{v}^{-1}(\mathrm{x}-\mathrm{a})\right]=\operatorname{Gamma}\left(\frac{1}{2}\right): \quad[10] 150$.

$\mathrm{D}$ (HM) : MR4:164.

$\mathrm{D}\left(\sqrt{\mathrm{x}^{2}+\mathrm{y}^{2}}\right), \mathrm{D}\left(\sqrt{\mathrm{x}^{2}+\mathrm{y}^{2}+\mathrm{z}^{2}}\right)$ in special circumstances: MR16:37\%.

$\mathrm{D}\left(\Sigma \mathrm{x}_{\mathrm{i}}^{2}\right): \quad[l] 3: 353$.

$\mathrm{D}\left(\chi^{2}\right)=\mathrm{D}\left(\sum \frac{\mathrm{x}_{1}-\mathrm{m}_{1}}{\sigma_{1}}\right)^{2}=\chi^{2}(\mathrm{n}): \quad[6] 10.3$.

$\mathrm{D}\left(\mathrm{s}^{2}\right)=$ Type III $\left(\mathrm{n} / 2 \mathrm{v}, \frac{1}{2} \mathrm{n}\right): \quad[d] 5: 281,[6] 10.3$.

$\mathrm{D}\left[(1 / \mathrm{n}) \Sigma\left(\mathrm{x}_{1}-\mathrm{x}\right)^{2}\right]=$ Type III $\left[\left(\frac{1}{2} \mathrm{nv}^{-1}, \frac{1}{2}(\mathrm{n}-1)\right]\right.$ : $[6] 10.4$.

$\mathrm{D}(\mathrm{s})$ for $\mathrm{n}=2,3: \quad[c] 11: 27 \%$

$\mathrm{D}(\mathrm{s} / \overline{\mathrm{x}})$, Coefficient of variation: $[d] \mathrm{y}: 129,[a] 94$ : $564,[a] 95: 695$.

$\mathrm{D}$ (range/s): $[d] 17: 366,[c] 31: 20$.

$\mathrm{D}\left[\left(2 \mathrm{~s}^{2}\right)^{-\frac{1}{2}}\right]$, "precision constant" = Type $\mathrm{V}$, moments, etc.: $[d] 3: 20,[a] 97: 132$.

$\mathrm{D}\left(\log \mathrm{s}^{2}\right): \quad[b] 8: 128$.

$\mathrm{D}\left(\mathrm{ns}^{2} \mathrm{v}^{-1}\right)=\chi^{2}(\mathrm{n}-1):[3] 115$

For unequal $\mathrm{v}$,

$\mathrm{D}\left[\mathrm{n}_{1}\left(\mathrm{n}_{2}-1\right) \mathrm{V}_{2} \mathrm{~s}_{1}^{2} / \mathrm{n}_{2}\left(\mathrm{n}_{1}-1\right) \mathrm{V}_{1} \mathrm{~s}_{2}^{2}\right]=$ Snedecor $\left(\mathrm{n}_{1}-1, \mathrm{n}_{2}-1\right)$ : [4]115, [10]197.

Testing and confidence intervals: $\quad[d] 13: 371$.

Power function: $[d] 17: 182$. 
For equal $v$

$\mathrm{D}\left(\mathrm{n}_{1} \mathrm{~s}_{1}^{2} \mathrm{v}^{-1}+\mathrm{n}_{2} \mathrm{~s}_{2}^{2} \mathrm{v}^{-1}\right)=\chi^{2}\left(\mathrm{n}_{1}+\mathrm{n}_{2}-2\right)$,

$$
D\left(\frac{1}{2} \log \frac{n_{1}\left(n_{2}-1\right) s_{1}^{2}}{n_{2}\left(n_{1}-1\right) s_{2}^{2}}\right)=\text { Fisher }\left(n_{1}-1, n_{2}-1\right) \text { : }
$$

$[10] 198$.

$\mathrm{D}\left(\mathrm{v}^{-1} \Sigma\left(\mathrm{n}_{1}-1\right) \mathrm{s}_{1}^{2}\right)=\chi^{2}: \quad$ [4]116.

$\mathrm{D}$ (variance ratio): $[k] 11: 136$.

Distribution of various statistics from $\mathrm{k}$ normal populations with common variance: $[e] 1 \%: 2$.

Ranking variances: $[g] 51: 621$.

Distribution of many quantities in a wide variety of cases: J. Soc. Stat. Paris 96:262.

$\mathrm{D}$ (various $\mathrm{Q}): \quad$ MR13:142.

$\mathrm{D}(\overline{\mathrm{x}}, \mathrm{s}): \quad$ [2]238, MR8:161.

$\mathrm{D}\left(\mathrm{b}_{2}\right)$ for $\mathrm{n}=4$ is hypergeometric: $[c] 25: 411$.

$\mathrm{D}\left(\mathrm{b}_{1}\right)$ for $\mathrm{n}=4$ is hypergeometric: [c]25:20\%, [c] $33: 68$.

$\mathrm{D}$ (midrange): $\quad[d] 21: 100$.

$\mathrm{D}$ (range): $[c] 17 \%: 364,[c] 18: 173,[c] 24: 404,[c] 39: 130$.

Quasi-range: $[d] 28: 179$.

$\mathrm{D}(\mathrm{r}): \quad[b] 15: 193$.

$\mathrm{D}(\xi): \quad[d] 26: 114$.

$\mathrm{D}(\mathrm{Q}): \quad$ MR22:60.

$\mathrm{D}\left(\mathrm{x}_{1} \mathrm{x}_{2}\right): \quad[d]^{\gamma}: 1,[d] 18: 265$.

$\mathrm{D}\left(\mathrm{x}_{1}, \ldots, \mathrm{x}_{\mathrm{n}}\right):$ [4]98.

$\mathrm{FD}(\mathrm{m})=\mathrm{N}\left(\overline{\mathrm{x}}, \mathrm{vn}^{-1}\right): \quad[c] 30: 401,414,[p] 17: 231$.

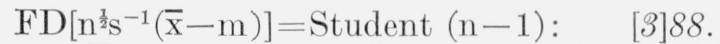

Tests: [3]98, [c]33:173, $[n] 5: 90,[d] 9: 279$, $[k] 6: 395$.

$\mathrm{FD}(\mathrm{m}, \sigma): \quad[3] 89,[k] 6: 395,[d] 10: 68$.

$\mathrm{FD}(1 / \sigma)=$ Helmert $(\mathrm{n}-1,1 / \mathrm{s} \sqrt{\mathrm{n}}): \quad$ [3]89.

A priori distributions of $\mathrm{m}$ and $1 / \sigma$ : MR9:48.

Ranking means: $[t] \%: 131$.

\section{Estimation}

C. - R. $(\mathrm{m})=\mathrm{vn}^{-1}$, i.e. $\overline{\mathrm{x}}$ efficient: [3]20, [y]11:182.

[1]483, [4]135,

$\operatorname{Var}(\overline{\mathrm{x}}) \leq \operatorname{var}(\xi): \quad$ [4]92.

$\operatorname{UMVUE}(\mathrm{m})=\overline{\mathrm{x}}: \quad[3] 51,[t] 4: 167$.

$\overline{\mathrm{x}}$ unbiased: $\quad[p]^{\mathrm{\gamma}} \mathrm{y}: 150$.

Minimax estimates of $\mathrm{m}: \quad[d] 21: 218,[d] 22: 28$.

Best "density unbiased" estimate of $\mathrm{m}: \quad$ [d]25:399.

$\sim$ efficiency of $\xi$ is $2 / \pi=.6366: \quad$ [1] $490,[u] 22: 706$.

Estimation of $\mathrm{m}$ when it must be integral, etc: [b]12:192.

Mean of $\mathrm{k}^{\text {th }}$ values from top and bottom has $\sim$ efficiency zero: $\quad[1] 490$.
C. - R. $(\sigma)=\mathrm{v}(2 \mathrm{n})^{-1}$, hence $\mathrm{s}$ is efficient:

$[2] 224$.

Efficiency of estimates of $\sigma: \quad[c] 37: 182$.

In estimating $\sigma$,

$\sqrt{\frac{1}{2} \mathrm{n}} \frac{\Gamma\left(\frac{1}{2} \mathrm{n}\right)}{\Gamma\left[\frac{1}{2}(\mathrm{n}+1)\right]} \sqrt{\frac{1}{\mathrm{n}} \Sigma\left(\mathrm{x}_{\mathrm{l}}-\mathrm{m}\right)^{2}}$ is more efficient than

$\sqrt{\frac{1}{2} \mathrm{n}} \frac{\Gamma\left[\frac{1}{2}(\mathrm{n}-1)\right]}{\Gamma\left(\frac{1}{2} \mathrm{n}\right)} \mathrm{s}: \quad[1] 484$.

C. - R. $(\sigma / \mathrm{m}): \quad[e] 8: 204$.

$[\mathrm{n} /(\mathrm{n}-1)] \mathrm{s}^{2}$ unbiased for $\mathrm{v}: \quad[p]^{7 \%}: 152$.

Estimation of $\sigma$ for industrial quality control:

$[g] 49: 375$.

Estimation of $\sigma$ from percentiles: $[i] 40: 85$.

Estimation of $\mathrm{v}$ and $\sigma: \quad[d] 18: 584,[b] 1: 78$.

C. - R. $(\mathrm{v})=2 \mathrm{v}^{2} \mathrm{n}^{-1}$, hence $\mathrm{s}^{2}$ efficient : $\quad[1] 484$.

$\operatorname{MLE}(\mathrm{v})=\mathrm{s}^{2}: \quad[6] 10.3$.

MLE(v) depends on whether m known: [3]34.

Estimation of $\mathrm{v}: \quad[e] 12: 5 \%,[i] 40: 85$.

In estimating $\mathrm{v}, \sim$ efficiency of $\mathrm{s}^{2}=+1: \quad[3]^{\mathrm{y}}$.

If $\mathrm{m}$ constant, $\mathrm{s}^{2}$ not sufficient: [3]11, [4]136.

In estimating $\mathrm{v}, \sim$ efficiency of mean deviation $=.876$ :

$[3] \%$.

Unbiased estimates: A. M. S. Translation No. 98.

Closest estimates of $\mathrm{m}, \mathrm{v}: \quad[u] 33: 45$.

Unbiased estimation of mean absolute deviation:

MR13:36\%.

In estimating $\mathrm{m}, \mathrm{v} \sim$ variance-covariance matrix:

$[4] 142$.

$\overline{\mathrm{x}}, \mathrm{s}^{2}$ are moments estimates of $\mathrm{m}, \mathrm{v}: \quad$ [1]498.

$\overline{\mathrm{x}},[\mathrm{n} /(\mathrm{n}-1)] \mathrm{s}^{2}$ are $\sim$ efficient: $[1] 494$.

$\operatorname{MLE}(\mathrm{v}, \mathrm{m})=\overline{\mathrm{x}}, \mathrm{s}^{2}: \quad[1] 504,[6] 156,[3] 20,34,[4] 132$.

$\overline{\mathrm{x}}, \mathrm{s}^{2}$ joint sufficient: [3]11, [4]136, [e]17:211, [i] $38: 181$.

Estimation of $\mathrm{m}, \sigma: \quad[d] 17: 386,[e] 10: 321,[e] 8: 12$.

Estimation: $\quad[c] 35: 186$.

Minimum $\chi^{2}$ estimation: $[c] 11: 262$.

MLE from censored sample: [i]32:124.

Censored sample: $\quad[c] 39: 260,[c] 43: 225$, MR14:569. MR15:241.

Estimation of $\mathrm{m}^{2}: \quad[u] 45: 214,[d] 1 \%: 43$

If $\mathrm{v}$ known, confidence regions for $\mathrm{m}$ are $\overline{\mathrm{x}} \pm \mathrm{k}_{\mathrm{p}} \mathrm{v}^{\frac{1}{2}} \mathrm{n}^{-\frac{1}{2}}$, where $\mathrm{k}_{\mathrm{p}}$ are the $\mathrm{p} \%$ values of the normal: [1]514.

Confidence intervals for $\mathrm{m}$ : [4]130, [6]224, [10]189.

Seq. confidence intervals for $\mathrm{m}: \quad[d] 18: 427,[b] 19$ : 133.

Confidence intervals for v: [4]131, [6]226.

Interval estimation of $\mathrm{v}$ and $\sigma: \quad[e] 6: 11 \%$. 
Confidence limits for $\mathrm{v}_{1} / \mathrm{v}_{2}$ : [4]131.

Confidence limits for $\mathrm{m}_{1}-\mathrm{m}_{2}$ with same $\mathrm{v}$ :

[4]130.

Interval estimation of $\mathrm{m}_{1}-\mathrm{m}_{2}$ (Behren's Problem): $[3] 91,[d] 18: 601,[d] 14: 35,[d] 15: 430,[d] 20: 616,[d] 21:$ $507,[e] 4: 39,108,[d] 24: 390[p] 7$ :232.

Confidence limits on $\mathrm{m}$ and $\mathrm{s}$ : [e]2:13, [o]8:83.

Confidence intervals for $\mathrm{m}, \mathrm{v}$ : [6]227, [3] 79 .

Tolerance limits: [d]13:398, [d]17:208, ( ) $[d] 17: 238,[d] 27: 171,[t] 1: 164$.

\section{Testing}

Testing $\mathrm{m}_{1}>\mathrm{m}_{2}: \quad[d] 14: 149$.

Tests on $\mathrm{m}$ : [6]259.

Unbiased regions for testing $\mathrm{m}_{1}=\mathrm{m}_{2}$ : $\quad$ [37320.

Power function of $\mathrm{m} \geq \mathrm{m}_{0}$ : $\quad$ [3]305.

Test of $\mathrm{m}$ using range in place of $\mathrm{s}: \quad[d] 17: 71$.

Hypotheses on m: [17533, [4]149.

Tests based on (rectans"ular a priori) distributions of $\mathrm{m}$ and $\mathrm{v}$ : $\mathrm{Z18:158.}$

Seq. tests on $\mathrm{m}: \quad[d] 16: 171,[e] 10: 364,368,[b] 9: 250$, $[c] 37: 334$.

Control charts on $\mathrm{m}: \quad[b] 16: 131$.

Testing $\mathrm{m}_{1}$ against $\mathrm{m}_{2}$ and $\sigma_{0}$ against $\sigma_{1}$ by quick counting methods: $[e] 17: 80$.

Seq. hypotheses on m: |d]20:502, $[g] 40: 303$.

Student's hypothesis: $[g] 31: 318$.

Student's is best for testing $\mathrm{m}_{1}=\mathrm{m}_{2}$ : [3]285, 291, [e]12:\%9.

LR test of $\mathrm{m}=\mathrm{m}_{0}$ is Student: [4]150.

Power function for Student test: $[d] 17 \% 192$.

Seq. Student test: $[c] 37: 326$.

Comparison of two means: [10]190, [c]38:252, $[c] 35: 88,[0] 4: 31$.

Comparison of k means: $\quad[t] \%: 1$.

Testing whether variance is constant: $[y] 20: 114$.

Three decision seq. test of $\mathrm{m}$ : [y]23:22, Amsterdam Mathematical Centre Stat. Dep. Rep SP34.

Testing whether many means are all zero: $[e] 8$ : $70,14] 176$.

Testing $\overline{\mathrm{x}}_{1}-\overline{\mathrm{x}}_{2}: \quad[i] 29: 21,[c] 41: 361$.

Testing $\overline{\mathrm{x}}_{1}-\overline{\mathrm{x}}_{2}$ without assuming $\mathrm{v}_{1}=\mathrm{v}_{2}$ : [13]433.

Linear hypotheses: $\quad[c] 2 \%: 161,[3] 292,300$.

Joint tests: $\quad[t] 6: 25,73$.

Testing outlying observations: $\quad[e] 17: 67$.

$\mathrm{H}: \sigma=\sigma_{0}: \quad[3] 28 \%,[d] 8: 193$.

$\mathrm{OC}$ for $\chi^{2}$ test of $\sigma=\sigma_{0}: \quad[d] 17: 179$.

Seq. test on $\sigma: \quad[e] 10: 369$.

Seq. test on $\sigma_{1}=\sigma_{2}: \quad[e] 12: 63,[b] 11: 101$.

Tests on v: $[6] 26 \%$.

Fisher is best for testing $\mathrm{v}_{1}=\mathrm{v}_{2}$ : [3]289

Testing homogeneity of variances: $[c] 31: 250$.

The most powerful test of

$$
\left\{\begin{array}{c}
\mathrm{H}: \quad \sigma=\sigma_{0}, \mathrm{~m} \\
\text { Alt: } \sigma=\sigma_{1}, \mathrm{~m}=\mathrm{m}_{1}
\end{array}\right\} \text { is } \Sigma\left(\mathrm{x}_{1}-\overline{\mathrm{x}}\right)^{2}<\mathrm{c} .
$$

Hypothesis of equality of many normal variances: $[b] 6: 89,[c] 29: 124$.

Significance of smallest of set of variances: $11 \%$.
Critical regions for $\mathrm{m}$ and $\mathrm{v}$ : $\quad[3] 278$.

Bibliography of testing equality of variances: $[a] 109: 45 \%$.

Seq. ratio test terminates: $\quad[e] 8: 342$.

OC function: $[g] 4^{7}: 191$.

Power functions of tests: $[d] 17: 189$.

Whether two samples are from the same normal population: $\lceil n] 7 \%: 3,[k] 17: 302$.

Tests for normality: [c]28:295, [c]27:310, 333, $[c] 34: 209,[0] 1: 125,[i] 20: 152, Z 19: 74$.

Tests of various composite hypotheses: $[e] 9: 30,[e] 10: 29$.

Decision problems: [b]15:55.

E. Miscellaneous

Independence of $\mathrm{x}$ and $\mathrm{s}$ (Student-Fisher Theorem): [i]19:108, J. Math. Soc. Jap. 1:111, MR1:346.

$\mathrm{s}^{2}, \mathrm{x}$ independent: [4]108, MR14:775.

Normality if and only if $\overline{\mathrm{x}}$ and $\mathrm{s}^{2}$ independent: [d]13:91, [d]16:400, [d]13:91, NBS Rep. 2267, J. Math. Soc. Jap. 1:111.

Normality if and only if $\left.\mathrm{D}(\overline{\mathrm{x}}, \mathrm{s}) \equiv \mathrm{L} \cdot \mathrm{sn}^{-2}: \quad \mid d\right] 14$ : $19 \%$.

Normality if $\mathrm{D}(\mathrm{x}) \mathrm{D}(\mathrm{y})=\phi\left(\sqrt{\mathrm{x}^{2}+\mathrm{y}^{2}}\right): \quad$ MR10:125.

Various characterizations of normality: [e]13:359, $[d] 28: 126, \mid i] 39: 59,[d] 27: 858,[e] 14: 180, A m . J$. Math. 61:726, Math. Z. 41:405, 213:214, 3rd Berkeley Symp. 2:195, MR16:1034.

Generalization of Student-Fisher theorem:

[i]20: 248.

Independence of quadratic forms: $[0] 1: 83$, Proc. Roy. Soc. Edin. 60:40, [u]30:178, [c]37:93, [c]14:195, [d]15:427, [d]20:119, MR12:509.

Bayes' theorem: $[n] 16-1: 113$.

Cochran's theorem: [4]10\%,68,

More generally: $\quad[d] 11: 100$.

History of normal: $\quad[c] 16: 402$.

History of distribution of $\mathrm{s}: \quad[c] 23: 416$.

Distributions which converge to normality: $[d]$ $10: 247$.

Discrete analog: $[c] 44: 365$.

Regression of $\mathrm{x}$ and $\mathrm{t}$, where $\mathrm{m}=\mathrm{a}-\mathrm{be}^{-\mathrm{kt}}: \quad[d] 18$ : 596.

Comparing percentage points of two normals: [d]19:93.

k samples: [3]295.

Truncated sample: [d]23:237.

Sampling from $\mathrm{N}\left(\Sigma \mathrm{a}_{\mathrm{k}} \mathrm{m}_{\mathrm{k}}, \mathrm{v}\right):$ [4]160

$\operatorname{Max} \Sigma \mathrm{x}_{\mathrm{i}}, \min \Sigma \mathrm{x}_{\mathrm{i}}: \quad[c] 40: 35$.

See also: $[d] 25: 389,[d] 1: 151,[d] 6: 197,[d] \%: 7 \%$, $[d] 10: 365,[d] 13: 235,[d] 17: 483,[d] 20: 123,[d] 21: 362$, $[d] 21: 55 \%,[d] 22: 596,[d] 23: 43,[d] 23: 384,[d] 23: 54 \%$, $[d] 25: 16,[l] 3: 309,[e] 9: 6,[13] 345,[c] 10: 522,[c] 13: 28 \%$, $[c] 16: 239,[c] 24: 184,[a] 79: 455,[f] 7: 23,[c] 31: 238,[c]$ $34: 61,98,[n] 5: 3,[n] 12-3: 65,[c] 40: 116,[c] 32: 226,301$, $[g] 48: 550,[d] 25: 636,698,[v] 5: 337,[g] 51: 88$, Brit. Assoc. Math Tables (3rd Ed.) v.I p. xxviii, N.B.S. Rep. 2545, C.R. Acad. Sci. Paris 238:444, [o]1:83, App. Sci. Research (Netherlands) Sect. A 3:297, J. Franklin Inst. 260:209, N.B.S. Rep. 2267, Am. J. Math. 57:821, Ann. Math. 35:312, Nat. Acad. Sci. 28:297, [y]24:2, Z18:225, MR16:52, MR14:1098, Z5:173, Z19:317, MR17:53, [w]7:193. 
1.2. Normal: $\mathrm{N}(\mathrm{O}, \mathrm{v})$

$\mathrm{D}(\mathrm{x})=(2 \pi \mathrm{v})^{-\frac{1}{2}} \exp \left(-\frac{1}{2} \mathrm{X}^{2} \mathrm{v}^{-1}\right)$ :

$[10] 50,[c] 31: 1$.

$\mathrm{Ch}(\mathrm{x})=\exp \left(-\frac{1}{2} \mathrm{vt}^{2}\right): \quad$ [2]94.

$\operatorname{MGF}(\mathrm{x})=\exp \left(\frac{1}{2} \mathrm{vt}^{2}\right): \quad$ [2]53.

$\mathrm{C}(\mathrm{x}): \quad[c] 25: 379$.

$\alpha_{2 \mathrm{k}}=\sigma^{2 \mathrm{k}}(2 \mathrm{k}) ! / 2^{\mathrm{k}} \mathrm{k} !: \quad[10] 54$.

2nd cumulant $=\mathrm{v}$, others zero: $\quad[2] 6 \%$.

Pearson type: [2]141.

$\mathrm{D}(\overline{\mathrm{x}})=\mathrm{N}(0, \mathrm{v} / \mathrm{n}): \quad[2] 175,[n] 10-3: 90$.

$\mathrm{D}(\mathrm{x} / \mathrm{y})=$ Cauchy: $[18] 1-150,[w] 1: 74$.

$\mathrm{D}\left(\mathrm{x}^{2}\right)=$ Type III: $\quad[i] 26: 212$.

$\mathrm{D}\left(\mathrm{s}^{2}\right): \quad$ [2]246, [u]28:456, Z15:118.

$\mathrm{D}\left(\Sigma \mathrm{x}_{\mathrm{i}}^{2} / 2 \mathrm{v}\right)=\operatorname{Gamma}\left(\frac{1}{2} \mathrm{n}\right)$.

$\mathrm{D}\left(\Sigma \mathrm{x}_{\mathrm{i}}^{2}\right)$ etc: $[c] 35: 4 \%$.

$\mathrm{D}\left(\Sigma \mathrm{x}_{\mathrm{i}}^{2}\right), \mathrm{D}\left(\mathrm{Q}_{1} / \mathrm{Q}_{2}\right): \quad[w] 1: 7_{4}$.

$\mathrm{D}(\mathrm{Q} / \mathrm{v})=\chi^{2}(\mathrm{r}), Q$ of rank $\mathrm{r} ; \neq 0$ eigenvalues all $+1:[d] 9: 43,[c] 25: 122.219: 357[c] 30: 407$.

$\mathrm{Ch}(\mathrm{Q})=\left[\Pi\left(1-2 \mathrm{itvk}_{\mathrm{i}}\right)^{\frac{1}{2}}\right]^{-1}$.

$\mathrm{D}\left[\mathrm{x} \mathrm{n}^{\frac{1}{2}}\left(\Sigma \mathrm{x}_{\mathrm{i}}^{2}\right)^{-\frac{1}{2}}\right]=$ Type II: $\quad$ [i]29:13.

$\mathrm{D}(\mathrm{x})$ assuming $\mathrm{v}$ is Type III: [d]28:510.

$\mathrm{D}(\mathrm{x} / \mathrm{s})=$ Student $($ testing $): \quad[c] 37: 65$.

$\mathrm{D}$ (range) for $\mathrm{n}=3$, unbiased critical region: 327 .

$\mathrm{FD}(\mathrm{v})=$ Type $\mathrm{V}\left[\Sigma \mathrm{x}_{\mathrm{i}}^{2} /(\mathrm{n}-2), \frac{1}{2}(\mathrm{n}-2)\right]$ :

C. - R. $(\mathrm{v})=2 \mathrm{n}^{-1} \mathrm{v}^{2}: \quad[1] 484,[p] \%: 159$.

$[p] \%: 226$.

If $\mathrm{D}(\mathrm{n})=$ No. 3.5: $\quad$ MR14:391.

$\operatorname{MLE}(\mathrm{v})=\mathrm{n}^{-1} \Sigma \mathrm{x}_{\mathrm{i}}^{2}: \quad$ [4]141.

$\mathrm{s}^{2}$ UMVUE of $\mathrm{v}$, but $\mathrm{s}$ not of $\sigma:$ [3]52,54.

Neyman-Pearson on hypothesis testing: [c]20: 178.

Most powerful test of $\left\{\begin{array}{c}\mathrm{H}: \sigma=\sigma_{0} \\ \text { Alt: } \sigma=\sigma_{1}\end{array}\right\}$ is $\overline{\mathrm{x}}^{2}+\mathrm{s}^{2}>\mathrm{c}$ : $[3] 275$.

Completeness: $\quad[e] 10: 313$.

Unbiased critical regions: [3]212.

Testing $\mathrm{s}_{1}^{2} / \mathrm{s}_{2}^{2}$ etc: $[e] 5: 15 \%$.

Testing serial correlation: $[i] 31: 103$.

Various devices for showing area $=+1: \quad[d] 5: 136$.

Inference: $[b] 15: 52$.

As "Maxwell-Bolzmann" distribution: [12]39.

Variance of Gini's mean difference is $\sim \mathrm{vn}^{-1}(.8068)^{2}$ : [2]21\%.

Mean difference: $\quad[c] 28: 432$.

Properties of $\mathrm{f}(\mathrm{x})$, where $\mathrm{x}$ is $\mathrm{N}(0, \mathrm{v}): \quad[c] 17: 211$.

See also: $[d] 12: 239,[c] 3: 311,[q] 26: 178,[c] 31$ : 260, $[n] 13-1: 51,[u] 30: 330$, MR9:364, MR3:2, [a]83: 127.

\subsection{Normal: $\mathrm{N}(\mathrm{m}, \mathrm{l})$}

$\mathrm{D}(\mathrm{x})=(2 \pi)^{-\frac{1}{2}} \exp \left[-\frac{1}{2}(\mathrm{x}-\mathrm{m})^{2}\right]$.

$\mathrm{E}\left(\mathrm{x}_{1}^{2}+\mathrm{x}_{2}^{2}\right)=\left(\frac{1}{2} \pi\right)^{\frac{1}{2}}, \operatorname{Var}\left(\mathrm{x}_{1}^{2}+\mathrm{x}_{2}^{2}\right)$

$=2-\frac{1}{2} \pi, \mathrm{E}|\mathrm{x}|=(2 / \pi)^{\frac{1}{2}}, \mathrm{E}\left(\mathrm{e}^{\mathrm{ax}}\right)$

$=\mathrm{e}^{\frac{1}{2} \mathrm{a}^{2}}, \operatorname{var}\left(\mathrm{e}^{\mathrm{ax}}\right)=\mathrm{e}^{2 \mathrm{a}^{2}}-\mathrm{e}^{\mathrm{a}^{2}}$ :

$[8] 120$.

$\mathrm{D}(\overline{\mathrm{x}}): \quad[3] 2$.

$\mathrm{D}\left(\mathrm{x}^{2}\right), \mathrm{D}\left(\mathrm{x}_{1}^{2}+\mathrm{x}_{2}^{2}\right), \mathrm{D}\left(\mathrm{x}_{1}^{2}+\mathrm{x}_{2}^{2}\right)^{\frac{1}{2}}: \quad[8] 95$.

$\mathrm{D}(\mathrm{xy})=$ Bessel: $\quad$ MR10:200.

$\mathrm{FD}(\mathrm{m})=\mathrm{N}\left(\overline{\mathrm{x}}, \mathrm{n}^{-1}\right):[3] 85$.
Bayes' distribution (m): [3]91.

$\overline{\mathrm{x}}$ sufficient: $\quad[3] 8,[e] 17: 211,[p]^{7} \mathrm{\gamma}: 161$.

$\overline{\mathrm{x}}$ is consistent: $\quad[3] 3,26$.

$\overline{\mathrm{x}}$ is MLE: [4]140, [c]33:125.

$\overline{\mathrm{x}}$ is minimax.

Efficiency of $\xi=.637: \quad[3] 6$.

Confidence intervals for $\mathrm{m}: \quad[3] 63,70,[p] 7$ :222.

MLE for $\chi^{2}$ test: [d]25:580.

Remarks on testing: $[d] 13: 62$.

Most powerful test of $\left\{\begin{array}{c}\mathrm{H}: \mathrm{m} \leq \mathrm{m}_{0} \\ \text { Alt: } \mathrm{m}=\mathrm{m}_{1} \mathrm{~m}_{0}\end{array}\right\}$

is $\overline{\mathrm{x}}>\mathrm{c}: \quad[3] 2 \% 4$.

UMP test of $\mathrm{m}=0: \quad[13] 452$.

Seq. test: $[c] 43: 452$.

Completeness: $\quad[e] 10: 313$.

Unbiased critical regions: [3]311.

Testing equality of several means: $[e] 8: 69$.

Testing; called "Laplace": [v]2:251.

Peculiar composite hypothesis on $\mathrm{m}$ : [3]306.

Inference: $[b] 15: 52$.

Pitman's method: [3]324.

Range: $\quad[c] 38: 463$.

See also: $[c] 27: 466,[c] 31: 202,[c] 36: 460,[g]^{7}: 95$ 3rd Berkeley Symposium 1:197.

\subsection{Normal: N $(0,1)$ (Gaussian)}

$\mathrm{D}(\mathrm{x})=(2 \pi)^{-\frac{1}{2}} \exp \left(-\frac{1}{2} \mathrm{x}^{2}\right): \quad[\mathrm{\gamma}] 129$.

General expose: Acta Math $7 \%: 1$.

$\mathrm{C}(\mathrm{x})$ as continued fraction: [2]130.

$\mathrm{C}(\mathrm{x})$ as a series: $\quad[c] 19: 13$.

Bounds on $\mathrm{C}(\mathrm{x}): \quad[c] 42: 263$.

Property of $\mathrm{C}(\mathrm{x})$ : $\quad$ Math. Zeit $41: 405$.

$\mathrm{C}(\mathrm{x}): \quad$ MR10:267.

$\sim \mathrm{C}(\mathrm{x}): \quad M R 16: 628$.

$\operatorname{MGF}(\mathrm{x})=\exp \left(\frac{1}{2} \mathrm{t}^{2}\right)$.

$\mathrm{Ch}(\mathrm{x})=\exp \left(-\frac{1}{2} \mathrm{t}^{2}\right): \quad[8] 16 \%,[1] 100$.

$\alpha_{2 \mathrm{k}}=(2 \mathrm{k}) ! / 2^{\mathrm{k}} \mathrm{k} !: \quad[d] 5: 32,[d] 11: 353,[h] 1: 13,193$, [1]208.

Absolute moments: $\quad$ Z1:26.

Median and quartiles: $[c] 25: 79$.

$\mathrm{D}(\mathrm{n} \overline{\mathrm{x}})=$ Normal: $\quad[c] 19: 22 \%$.

$\mathrm{D}\left(\mathrm{s}^{2}\right): \quad[e] 5: 138$.

$\mathrm{D}\left(\mathrm{x}^{2}\right): \quad[d] 1: 340,[e] 5: 138$.

$\mathrm{D}\left(\Sigma \mathrm{x}_{\mathrm{i}}^{2}\right)=\chi^{2}(\mathrm{n}): \quad$ [2]231, [4]103, [9]331.

$\mathrm{D}\left(\Sigma \mathrm{k}_{\mathrm{i}} \mathrm{x}_{\mathrm{i}}\right): \quad[d] 13: 17$.

$\mathrm{C}\left(\mathrm{n}^{\frac{1}{2}} \mathrm{~s}^{-1} \overline{\mathrm{x}}\right)=$ Student: $\quad$ [9]336. 
$\mathrm{D}(\mathrm{Q}), \mathrm{D}\left(\mathrm{Q}_{1} / \mathrm{Q}_{2}\right): \quad[e] 17: 37$.

$\mathrm{D}\left[\Sigma\left(\mathrm{x}_{1}-\overline{\mathrm{x}}\right)^{2}\right]=\chi^{2}(\mathrm{n}-1): \quad$ [9]333.

$\mathrm{C}(\mathrm{r})$ expressed as an integral: [9]339.

$\mathrm{D}\left(\mathrm{x}_{1} \mathrm{x}_{2}\right)$ by Mellin transformation: $[d] 19: 375$.

$\mathrm{D}\left(\mathrm{x}_{1} / \mathrm{x}_{2}\right)=$ Cauchy: $\quad[d] 19: 375$.

$\mathrm{D}(\overline{\mathrm{x}}, \mathrm{s}): \quad[0] \gamma: 65$.

$\mathrm{D}$ (range) for $\mathrm{n}=3, \sim \mathrm{D}$ (range): $\quad[c] 34: 111$, $[c] 5: 313,[o] 8: 155,[c] 36: 142$.

Moments of sample median: $[d] 26: 600$.

$\mathrm{D}$ (extreme deviate): $[c] 35: 120$.

$\mathrm{C}$ (range) : $\quad[c] 32: 341$.

$\mathrm{D}\left(\Sigma \mathrm{x}_{i}^{2} / \Sigma \mathrm{y}_{\mathrm{j}}^{2}\right)=$ Snedecor: $\quad[d] 19: 9 \% 8$.

Ch fcns of estimates of $\mathrm{v}: \quad[d] 19: 25 \%$.

Generating functions: Z2:200.

Estimation of dispersion: $[c] 36: 96$.

Estimation of mean deviation: $[c] 33: 254,[c] 35: 304$.

Variance of median: $\quad[c] 23: 361$.

Testing $\mathrm{N}(0,1)$ against various alternatives: $[c] 30: 139$.

Multivariate analysis: $[3] X X V I I I$.

Limit of binomial: $\quad[7] 134$.

Central limit theorem: $\quad[i] 27: 139,[i] 29: 206$.

$\mathrm{K}^{\text {th }}$ value from the top: [1]3\% .

Censored samples: $[c] 41: 230$.

Ordered samples: [e]11:23.

Stratified sampling: $\quad[d] 5: 138$.

Variance in two samples: $[n] 13-3: 49$.

Ratio of two ranges: $[d] 21: 112$.

Tetrachloric functions: $[c] 14: 15 \%$.

Approximations: $[d] 17: 363$.

Sheppard's tables: $[c] 2: 1 \%$.

Grouping: [i]32:135.

Moments of order statistics: $[d] 41: 200$.

Occasionally called Laplace-Gauss, or even Laplace: Acta Math. 77:1, C. R. Acad. Sci. Paris 232:1999.

See also: $[d] 4: 109,[d] 17: 350,[d] 22: 425,[d] 24:$ 133, [d]24:297, [13]63, [c]18:395, [c]24:98, [c]24:280, $[c] 25: 195,[i] 6: 209,[17]{ }^{2} 0.41,[m] 6: 120,[d] 22: 418$, [y]24:22, [u]29:231, Z4:66, Z8:266, Z20:39, 145, MR12:191, Z18:412, MR17:756, C. R. Acad. Sci. Paris 238:444, Phil. Trans. Roy. Soc. London A237: 231, $M R^{7}: 18,25: 366,[y] 4: 189$.

\subsection{Truncated Normal}

$\mathrm{C}(\mathrm{x}): \quad[1] 248,[6] 243$.

Introduction, estimation, examples: $[15] 144$.

$\mathrm{D}\left(\Sigma \mathrm{x}_{\mathrm{i}}\right): \quad[b] 8: 223$.

Fitting: $\quad[c] 39: 252$.
Estimating $\mathrm{m}$ and $\mathrm{v}: \quad[g] 44: 518,[g] 47: 45 \%,[f] 9: 489$, $[0] 3: 37, M R 7: 461[c] 40: 52$.

Distribution of estimate of $\sigma: \quad$ [15]316.

Censored sample: $[c] 42: 516$.

MLE: [i]32:119.

See also: $[d] 9: 66,[d] 20: 458,[g] 47: 379$, Brit. Assoc. Math. Tables (3rd Ed.) v. 1 p. xxxv, MR2:231.

\subsection{Generalized Normal (Kapetyn)}

$(2 \pi \mathrm{v})^{-\frac{1}{2}} \exp \left[-\frac{1}{2} \mathrm{v}^{-1}(\mathrm{f}(\mathrm{x})-\mathrm{m})^{2}\right] \mathrm{df}(\mathrm{x}):$
C. $-\mathrm{R} \cdot(\mathrm{m})=\mathrm{v} / \mathrm{n}, \mathrm{C} \cdot \mathrm{R}-\mathrm{R} .(\mathrm{v})=2 \mathrm{v}^{2} / \mathrm{n}:$
$\operatorname{MLE}(\sigma)=\left[\mathrm{n}^{-1} \Sigma\left(\mathrm{f}\left(\mathrm{x}_{\mathrm{i}}\right)-\mathrm{m}^{2}\right]^{\frac{1}{2}}\right.$,
$\operatorname{MLE}(\mathrm{m})=\mathrm{n}^{-1} \Sigma \mathrm{f}\left(\mathrm{x}_{\mathrm{i}}\right)$.

See also: $[c] 5: 168$.

\subsection{Normals Added}

$\mathrm{D}(\mathrm{x})=(\mathrm{l}+\mathrm{k})^{-1}\left\{(2 \pi)^{-\frac{1}{2}} \exp \left(-\frac{1}{2}\left(\mathrm{x}+\mathrm{m}_{1}\right)^{2}+\mathrm{kv}^{-\frac{1}{2}}(2 \pi)^{-\frac{1}{2}}\right.\right.$ $\left.\exp -\frac{1}{2}\left(\mathrm{x}-\mathrm{m}_{2}\right) \mathrm{v}^{-1}\right\}: \quad[d] 2: 340,\left[h^{\prime}\right] 2: 63$.

$\operatorname{Var}(\mathrm{x})=(\mathrm{l}+\mathrm{k})^{-1}\left[1+\mathrm{m}_{1}^{2}+\mathrm{k}\left(\mathrm{v}+\mathrm{m}_{2}^{2}\right)\right]$.

Method for partition with example: $[s] 5: 4 \%$.

Semi-invariants: $\quad[i] 1 \%: 1$.

$\mathrm{D}(\overline{\mathrm{x}}): \quad[d] 11: 219$.

Three normals added: [d]5:237.

More generally: $[d] 3: 1,[d] 5: 230,[c] 3: 85$, MR14: 485 .

Sampling theory: $[n] 8-3: 6 \%$.

Many normals added: $\quad[c] 37: 429$.

Called "compound normal": [i] $32: 180$.

Bivariates: $[f] 8: 328$.

See also: $\quad[c] 40: 460,[e] 14: 369, M R 11: 258, M R 17$ 1102.

\subsection{Lognormal (a, m, v)}

$\mathrm{D}(\mathrm{x})=(\mathrm{x}-\mathrm{a})^{-1}(2 \pi \mathrm{v})^{-\frac{1}{2}} \exp \left[-\frac{1}{2} \mathrm{v}^{-1}(\log (\mathrm{x}-\mathrm{a})-\mathrm{m})^{2}\right]$, parameters and moments: [15]160, [5]121, [d]3:45. $[1] 258,[c] 4: 194$.

Graphical determination of parameters: $\left.\quad{ }_{i}^{\mathrm{r}} w\right] 9: 102$. Mean $=\mathrm{a}+\mathrm{e}^{\mathrm{m}+\frac{1}{2} \mathrm{v}}, \quad \operatorname{var}=\mathrm{e}^{2 \mathrm{~m}+\mathrm{v}}\left(\mathrm{e}^{\mathrm{v}}-1\right)$.

Another form

$$
\begin{aligned}
\mathrm{D}(\mathrm{x}) & =\frac{1}{\sqrt{2} \mathrm{c}(\mathrm{x}-\mathrm{a})} \exp \left\{-\frac{1}{2 \mathrm{c}^{2}}\left[\log \frac{\mathrm{x}-\mathrm{a}}{\mathrm{b}}\right]^{2}\right\} \\
\mathrm{m} & =\mathrm{be}^{\frac{1}{2} \mathrm{c}^{2}}+\mathrm{a}, \text { mode }=\mathrm{be}^{-\mathrm{c}^{2}}+\mathrm{a}, \mathrm{GM}=\xi \\
& =\mathrm{a}+\mathrm{b}, \text { moments, tables, regression, } \\
& \text { examples, bibliography: }[d] 4: 30,[b]^{y}: 155 .
\end{aligned}
$$

Moments, transformations: $\quad[w]^{7}: 152,[w] 8: 83$.

Complete treatment with bibliography: Aitchison, J. and Brown, J. A. C., "The Lognormal Distribution" Cambridge, 1957 (MR18:957).

Estimation of $\mathrm{m}: \quad[e] 10: 341$.

MLE: $\quad[g] 46: 206$, Intl. Congr. Math (1950) 1:581. 
Regression: $\quad[d] \%: 196$.

$\sim$ Tests on $\mathrm{m}: \quad[d] 28: 1044,[d] 27: 670$.

Called "Galton-Macalister": [c]32:239.

Called Gibrat: Kendall and Buckland, "A dictionary of Stat. terms."

Used to approximate Fisher distribution: 12:448.

Deduced from hypothesis about errors, etc.: 28:141.

$(\mathrm{x}-\mathrm{a}) /(\mathrm{b}-\mathrm{x})$ lognormal: $[17]$ No.46.

Transformation: $[c] 36: 155$.

Versus normal: Geochimica et Cosmochrmica Acta 8:53.

Discrete lognormal: $\quad[c] 37: 362$.

Compared with normal by means of Galton-Kapetyn apparatus: $[s]]_{4}: 129$.

Truncated lognormal: $\quad[i] 28: 150,[c] 38: 414$.

Lognormal $(0,0,1), \mathrm{E}(\mathrm{x})=\mathrm{e}^{\frac{1}{2}}, \mathrm{v}=\mathrm{e}^{2}-\mathrm{e}: \quad$ [8]120, $176,[17]$ No. 45, $[c] 22: 109,[d] 4: 30$.

See also: $[d] 14: 120,[1] 13: 161,[e] 12: 121,[b] 6$ : $174,[b] 11: 19,[d] 15: 182,[c] 4: 179,[c] 22: 146,[g] 34:$ 762, [g] $36: 493,[f] 1: 5 \%,[c] 36: 155,[c] 38: 427,[g] 48:$ 600, J. Franklin Inst., 244:471, 250:339, 250:419, 251: 499, 251:617, [g]50:904, $[c] 43: 404,[a] 119: 15 \%$, 185, 250:339, Ind. and Eng. Chem. 40:2289, J. Roy. Soc. (A)216:309, J. Phys. Chem., 56:442, [y]13:29, J. Hygiene 42:328, Z10: 173, MR3:4, Nature $156: 463$

\subsection{Wrapped-up Normal}

$\mathrm{D}(\mathrm{x})=\mathrm{k} \Sigma \mathrm{e}^{-\mathrm{c}(\mathrm{x}+\mathrm{j}) 2}: \quad$ Bull. Soc. Math. France 66: 32, 67:1, C.R. Soc. Math. France (1938) p 34, Ann. Ecole. Norm. Sup. 45:1 [t] 55:335, [d]18:589, Handbuch der Physik, Berlin, Springer 3:47\%.

\subsection{Gram-Charlier}

Two-term $\mathrm{D}(\mathrm{x})=(2 \pi)^{-\frac{1}{2}}\left[1-\mathrm{k} / 6\left(3 \mathrm{x}-\mathrm{x}^{2}\right)\right] \exp \left(-\frac{1}{2} \mathrm{x}^{2}\right)$ : [3]103, $13 \%$.

General Gram-Charlier: [4] $7 \%$.

$\mathrm{D}(\overline{\mathrm{x}}): \quad[d] 1: 199,[d] 2: 99$.

$\mathrm{D}\left(\mathrm{x}^{2}\right): \quad[i] 26: 212$.

$\mathrm{D}(\mathrm{s}): \quad[d] 6: 12 \%$.

t-test: $\quad[i] 26: 210$.

$\mathrm{D}(\mathrm{x})=\mathrm{f}(\mathrm{x}) \mathrm{N}(\mathrm{m}, \mathrm{v}): \quad[d] 23: 467,[g] 26(P): 233$.

$\mathrm{D}$ (various statistics): $[\mathrm{g}] 4: 1$.

Log Gram-Charlier: [i]28:145.

Type B Gram-Charlier: [d]8:183, [d]18:5\% 4, Trans. Amer. Math. Soc. 67:206, [d] 20:376, [i] $5: 1 \%$.

MGF factorial moments: $\quad$ Z5:219.

See also: $[a] 88: 576,[a] 89: 129,[c] 33: 126,[c] 36:$ $42 \%,[c] 38: 58, \quad 8 \%,[c] 39: 425,[i] \%: 14 \%,[l] 23: 283$, T.A.M.S. 67:206, Z18:320, Z22:243, Z2:43
1.11. Bivariate Normal $\mathrm{N}\left(\begin{array}{l}\left.\mathrm{m}_{1}, \mathrm{v}_{1} \rho\right) \\ \mathrm{m}_{2}, \mathrm{v}_{2}\end{array}\right)$

$\mathrm{D}(\mathrm{x}, \mathrm{y})=\left[2 \pi \sigma_{1} \sigma_{2}\left(1-\rho^{2}\right)^{\frac{1}{2}}\right]^{-1} \quad \exp \left\{-\frac{1}{2}\left[\mathrm{v}_{1} \mathrm{v}_{2}\left(1-\rho^{2}\right)\right]^{-1}\right.$ $\left.\left[\left(\mathrm{x}-\mathrm{m}_{1}\right)^{2} \mathrm{v}_{2}-2 \rho \sigma_{1} \sigma_{2}\left(\mathrm{x}-\mathrm{m}_{1}\right)\left(\mathrm{y}-\mathrm{m}_{2}\right)+\left(\mathrm{y}-\mathrm{m}_{2}\right)^{2} \mathrm{v}_{1}\right]\right\}$ $[6] 165,[5] 89,[4] 60$.

Introduction, properties, examples: $\quad$ [15]585.

Another form (Koopman-Darmois): [e]8:322.

$\mathrm{Ch}(\mathrm{x}, \mathrm{y})=\exp \left\{\mathrm{i}\left(\mathrm{m}_{1} \mathrm{~s}+\mathrm{m}_{2} \mathrm{t}\right)-\frac{1}{2}\left(\mathrm{v}_{1} \mathrm{~s}^{2}+2 \rho \sigma_{1} \sigma_{2} \mathrm{st}+\mathrm{v}_{2} \mathrm{t}\right)\right\}:$ [1]28\%.

MGF: $[6] 16 \%$.

$\mathrm{D}\left(\overline{\mathrm{x}}_{1}, \overline{\mathrm{x}}_{2}\right)=$ Normal bivariate: [4]101.

$\mathrm{D}(\overline{\mathrm{x}}-\overline{\mathrm{y}}): \quad[c] 2: 379$.

$\mathrm{D}(\mathrm{y} / \mathrm{x}): \quad[c] 24: 428$.

$\mathrm{D}(\mathrm{xy})$ and $\mathrm{D}(\mathrm{x} / \mathrm{y}): \quad[18] 1-151$, Am. Math. Monthly 49:26.

$\mathrm{Ch}(\mathrm{xy}): \quad[a] 42: 82$.

$\mathrm{D}(\mathrm{r}): \quad$ [4]120.

If $\rho=0, \mathrm{D}\left(\mathrm{r}^{2}\right)=\mathrm{B}(1, \mathrm{n}-2): \quad[10] 160,178$.

If $\rho=0, \mathrm{D}(\mathrm{b})=\mathrm{B}(1, \mathrm{n}-1): \quad[10] 180$.

$\mathrm{D}($ correlation ratio $)=$ No. 5.3: $\quad[10] 181$.

$\sim \mathrm{D}(\mathrm{r}): \quad[c] 10: 50 \%$.

$\mathrm{D}\left(\mathrm{rs}_{1} \mathrm{~s}_{2}, \mathrm{~s}_{1}^{2}, \mathrm{~s}_{2}^{2}\right): \quad[u] 29: 264$.

Distribution of various statistics: $[e] 1 \%: 21$.

$\operatorname{MLE}\left(\mathrm{m}_{1}, \mathrm{~m}_{2}, \mathrm{v}_{1}, \mathrm{v}_{2}, \rho \sigma_{1} \sigma_{2}\right)=\overline{\mathrm{x}}, \overline{\mathrm{y}}, \mathrm{s}_{1}^{2}, \mathrm{~s}_{2}^{2}, \mathrm{rs}_{1} \mathrm{~s}_{2}: \quad[3] 3 \%$.

$\operatorname{Var}(\overline{\mathrm{x}})=\mathrm{v}_{1} / \mathrm{n}, \operatorname{var}\left(\mathrm{s}_{1}^{2}\right)=\mathrm{v}_{1} / 2 \mathrm{n}, \operatorname{var}(\mathrm{r})=\mathrm{n}^{-1}\left(1-\rho^{2}\right)^{2}$, $\operatorname{cov}\left(\mathrm{s}_{1}, \mathrm{~s}_{2}\right)=\rho^{2} \sigma_{1} \sigma_{2} / 2 \mathrm{n}, \operatorname{cov}\left(\mathrm{r}, \mathrm{s}_{1}\right)=\rho \sigma_{1}\left(1-\rho^{2}\right) / 2 \mathrm{n}$, $\operatorname{cov}(\overline{\mathrm{x}}, \overline{\mathrm{y}})=\rho \sigma_{1} \sigma_{2} / \mathrm{n}: \quad[3] 38$.

MLE from fragmentary information: $\quad[d] 3: 163$.

$\bar{x}, \bar{y}$ are joint efficient in estimating $m_{1}$ and $m_{2}$; $\bar{x}, \bar{y}, \frac{n s_{1}^{2}}{n-1}, \frac{n s_{2}^{2}}{n-1}, \frac{n}{n-1} \mathrm{rs}_{1} s_{2}$ have joint efficiency $(\mathrm{n}-1 / \mathrm{n})^{3}: \quad[1] 495-6$.

$\left[\left(\mathrm{x}-\mathrm{m}_{1}\right) / \sigma_{1}\right]+\left[\left(\mathrm{y}-\mathrm{m}_{2}\right) / \sigma_{2}\right]$ and $\left[\left(\mathrm{x}-\mathrm{m}_{1}\right) / \sigma_{1}\right]$

$-\left[\left(\mathrm{y}-\mathrm{m}_{2}\right) / \sigma_{2}\right]$ are independent and $\mathrm{N}(0,2(1+\rho))$, $\mathrm{N}(0,2(1-\rho))$ respectively: $[6] 21 \%$.

Estimation: $[d] 1 \%: 395,[e] 8: 322$.

Estimation, testing: $\quad[15] 606,[g] 50: 884$.

Censored samples: $[x] 6: 83$.

Distribution ratio standard deviations: $[x] 6: 93$.

Confidence limits for r: $\quad[c] 29: 15 \%, J$. Nat. Inst. Personnel Res. 6:153.

Confidence limits for $\mathrm{m}_{1} / \mathrm{m}_{2}$ : [d]13:440, MR13: 962.

Sufficient statistics: $\quad[e] 17: 212$.

Comparison of two correlations: [10]203.

Tests of seven hypotheses on the parameters: $[d] 11: 410$.

Testing equality of two r's: [d]12:279.

Some tests: $\quad[w] \%: 46$. 
Testing equality of variances: raphy), [a]109:462.

$\mathrm{r}_{1}-\mathrm{r}_{2}: \quad[c] 25: 102$.

Sequential tests of $\rho: \quad[w] 8: 202$.

Truncation: MR2:231.

Fisher's original work on $\mathrm{r}$ and $\rho: \quad[n] 1-4: 1$.

Sufficient conditions for normal bivariate: 399, MR15:805.

k samples: $\quad[c] 27: 145,227$.

$\mathrm{x} / \sigma_{1}+\mathrm{y} / \sigma_{2}$ and $\mathrm{x} / \sigma_{1}-\mathrm{y} / \sigma_{2}$ are independent normal variables: $\quad[c] 31: 9$.

If $\rho=0$ then $\left(\mathrm{v}_{1} / \mathrm{v}_{2}\right) /\left[\left(\mathrm{v}_{1} / \mathrm{v}_{2}\right)+\left(\mathrm{s}_{1}^{2} / \mathrm{s}_{2}^{2}\right)\right]$ is Beta : $[c] 31: 10$, Called Bravais distribution: $[i] 19: 3$

Properties: $\quad$ Z15:310, MR1:246.

See also: $[d] 4: 196,[d] 14: 141,[c]^{7}: 260,[g] 26: 129$, [c]22:1, [c]25:356, [c]25:392, [f]8:328, [n] $9-3: 90,[c] 39:$ 238, [i]27:221, [6]218, [v]5:311, [d]27:1075, [c]44:289, $[x] 4: 85$, Harvard Ed. Rev. 1946, p. 52, MR4:280, MR8:283, MR14:1102, MR7:212, [y]1(No. 4) 20.

\subsection{Bivariate Normal $\mathrm{N}\left(\begin{array}{l}0, \mathrm{v}_{1,} \rho \\ 0, \mathrm{v}_{21}\end{array}\right.$}

$\mathrm{D}(\mathrm{x}, \mathrm{y})=\left(2 \pi \mathrm{M}^{\frac{1}{2}}\right)^{-1} \exp \left[-\frac{1}{2} \mathrm{M}^{-1}\left(\mathrm{x}^{2} \mathrm{~V}_{2}-2 \rho \sigma_{1} \sigma_{2} \mathrm{xy}+\mathrm{y}^{2} \mathrm{v}_{1}\right)\right]$ where $\mathrm{M}=\left|\begin{array}{cc}\nabla_{1} & \rho \sigma_{1} \sigma_{2} \\ \rho \sigma_{1} \sigma_{2} & \mathrm{v}_{2}\end{array}\right|=\mathrm{v}_{1} \mathrm{v}_{2}\left(1-\rho^{2}\right) ; \quad \mathrm{Ch}(\mathrm{x}, \mathrm{y})$

$=\exp \left(-\frac{1}{2} \mathrm{n}^{-1}\left(\mathrm{v}_{1} \mathrm{~s}^{2}+2 \rho \sigma_{1} \sigma_{2} \mathrm{st}+\mathrm{t}^{2} \mathrm{v}_{2}\right)\right]: \quad$ [9]308, [2] 22, 334, [4]60, [8]116, [15]588, [10]95, 106, [c]30:8. $\alpha_{40}=3 \mathrm{v}_{1}^{2}, \quad \alpha_{31}=3 \rho \sigma_{1}^{3} \sigma_{2}, \quad \alpha_{22}=\left(1+2 \rho^{2}\right) \mathrm{v}_{1} \mathrm{v}_{2}: \quad$ [2] 80 , [4]60.

Moments: $\quad[0] 3: 2,[c] 12: 177$.

Central moments: $\left[h^{\prime}\right] 4: 73$.

Incomplete moments: $[c] 13: 401,[c] 40: 22$.

Product-moments: $\quad[c] 12: 86$.

As limit of binomial: $\quad[a] 91: 548$.

If $\rho=0, \quad \sigma_{1}=\sigma_{2}$ called "circular normal," D(r), properties: $\quad[c] 29: 13 \%$.

$\mathrm{C}(\mathrm{x}, \mathrm{y})$ with other properties: $\quad[c] 33: 59,[c] 38: 475$.

Cumulants in terms of moments: [2]89.

$\operatorname{Var}(\mathrm{r})=\mathrm{n}^{-1}\left(1-\rho^{2}\right)^{2}: \quad$ [2]336.

$\operatorname{Var}(\mathrm{b})=\mathrm{n}^{-1} \mathrm{v}_{1}{ }^{-1} \mathrm{v}_{2}\left(1-\rho^{2}\right): \quad$ [2]337.

Marginal and conditional distributions: [4]62.

Regression: [3]144.

Correlation and regression with generalization: [i]24:1.

Contour ellipses: $\quad[s] 1: 203$.

Bilinear forms: $\quad[o] 1: 103,[d] 18: 565$.

Quadratic forms: $[d] 14: 195$.

$\mathrm{Ch}\left(\mathrm{x}^{2}, \mathrm{y}^{2}\right): \quad$ [2]336.

$\mathrm{D}($ variance-covariance $)=$ Wishart : $\quad$ [2]340, [1]29.6.
$\mathrm{D}(\mathrm{r}): \quad$ [2]342.

$\mathrm{D}(\mathrm{r})$ for $\mathrm{n}=4: \quad[u] 26: 536$.

$\mathrm{D}(\mathrm{b})=$ Type VII: $\quad$ [1]402.

$\mathrm{D}\left(\mathrm{s}_{1} / \mathrm{s}_{2}\right)=$ No. 8.3 if $\sigma_{1}=\sigma_{2}: \quad[e] 2: 65,[c] 31: 9$.

$\mathrm{D}\left(\mathrm{e}^{\mathrm{x}}, \mathrm{e}^{\mathrm{y}}\right): \quad[w] 2: 155$.

When, further, $\rho=0$ generalized Student: 30:190.

Simple function of $\mathrm{x} / \mathrm{y}$ is normal: $\quad[a] 93: 442$.

$\mathrm{D}(\mathrm{y} / \mathrm{x}): \quad[c] 32: 16,[i] 20: 61$.

$\mathrm{D}(\mathrm{xy}), \mathrm{D}(\mathrm{x} / \mathrm{y}): \quad$ MR3:171.

Joint distribution of Pearson betas: $\quad[c]^{7}: 386$.

$\mathrm{D}\left(\mathrm{s}_{1}^{2}, \mathrm{~s}_{2}^{2}, \mathrm{rs}_{1} \mathrm{~s}_{2}\right)$ etc: $[d] 5: 283$.

$\mathrm{D}\left(\mathrm{s}_{1}^{2}, \mathrm{~s}_{2}^{2}\right): \quad[e] 5: 139,[c] 25: 126$.

$\mathrm{D}\left(\frac{(\mathrm{n}-1)^{\frac{1}{2}}(\mathrm{~b}-\beta) \sigma_{1}}{\sqrt{\left(\mathrm{v}_{2}-\mathrm{b}^{2} \mathbf{v}_{1}\right)}}\right)=$ Student $(\mathrm{n}-1)$.

$\mathrm{D}\left(\frac{(\mathrm{n}-2)^{\frac{1}{2}}(\mathrm{~b}-\beta) \mathrm{s}_{1}}{\sqrt{\mathrm{v}_{2}-\mathrm{bv}_{1}}}\right)=$ Student $(\mathrm{n}-2)$ :

[9]5.13, [2] $348,[3] 156$.

Moments of dist. of covariance from

$\mathrm{N}\left(\begin{array}{c}0,1 \\ 0,1, \rho\end{array}\right): \quad[3] 334$.

$\mathrm{D}($ radial standard deviation $)=\mathrm{D}\left[\mathrm{n}^{-1} \Sigma\left(\mathrm{x}_{1}-\overline{\mathrm{x}}\right)^{2}+\Sigma\right.$ $\left.\left(\mathrm{y}_{1}-\overline{\mathrm{y}}\right)\right]^{\frac{1}{2}}: \quad[d] 15: 75$.

D (sample covariance): [3]359, [10]138.

$\operatorname{MLE}(\rho): \quad[3] 33$.

Confidence intervals for $\rho$ : $\quad[3] 81$.

$\operatorname{MLE}\left(\mathrm{v}_{1}, \mathrm{v}_{2}, \rho\right): \quad$ [2]339.

Estimation of $\rho$ when $\mathrm{v}_{1}=\mathrm{v}_{2}: \quad[d] 9: 149$.

Estimation of $\rho$ by rank correlation: $[d] 7: 40$, $[c] 40: 419$.

Truncation: $\quad[d] 21: 272$. and estimation: [e]12:277, MR16:498.

Testing $\mathrm{v}_{1} / \mathrm{v}_{2}$ : [3]138.

Testing $\sigma_{1} / \sigma_{2}$ and $\rho$ : [e]5:151.

Test whether two samples are from same population: [3]140.

Hotelling's generalized $\mathrm{T}$ applied to tolerance limits: $\quad[d] 14: 90$.

Odd fact for $\rho=0: \quad[d] 18: 442$.

See also: $[o] 12: 90,[c] 2: 369,[c] 4: 498,[c] 17: 176$, $[c] 20: 295,[g] 27: 254,[a] 83: 128,[c] 29: 74,[n] 2: 640$, $[n]^{7}: 6,[c] 32: 196,[c] 38: 3 \% 1,[i] \%: 220,[i] 24: 17,[n] 13-1:$ 21, 65, [u]28:457, Z12:267.

\subsection{Trivariate Normal}

$\mathrm{D}(\mathrm{x}): \quad[10] 255,260$.

Moments: $[0] 4: 15,[c]<0: 23$. 
Correlation: $\quad[i] 14: 158$.

Partial correlation: $[c] 10: 391$.

Yielding $2 \times 2 \times 2$ table: $\mid e] 10: 272$.

Student test for partial correlation: [10]256.

Snedecor test for multiple correlation: [10]25\%.

See also: $[d] 8: 179,[d] 12: 94,[n] 1-1: 151,[u] 44:$ 342, MR15:805.

\subsection{Multivariate Normal}

$\mathrm{D}(\mathrm{x})=\mathrm{Ce}^{-\mathrm{Q}}=\left[(2 \pi)^{\frac{1}{2} \mathrm{n}} \sigma_{1} \ldots \sigma_{\mathrm{n}}\right]^{-1}\left(\mid \mathbf{A}^{\frac{1}{2}}\right) \exp \left\{-\frac{1}{2} \Sigma \Sigma\left(\mathrm{a}_{\mathrm{ij}} \mid\right.\right.$ $\left.\left.\sigma_{1} \sigma_{j}\right)\left(x_{i}-m_{i j}\right)\left(x_{j}-m_{j}\right)\right\}$ where $A=|| a_{1 j}\|=\| \rho_{1 j} \|^{-1}$ =inverse of correlation matrix: [6]17\%, [2]376, [4]63.

$\mathrm{C}(\mathrm{x}): \quad[c] 40: 458,[c] 41: 351$.

$\mathrm{Ch}(\mathrm{x})=\exp \left\{\mathrm{i} \Sigma \mathrm{m}_{\mathrm{j}} \mathrm{t}_{\mathrm{j}}-\frac{1}{2} \Sigma \Sigma \rho_{\mathrm{ij}} \sigma_{\mathrm{i}} \sigma_{\mathrm{j}} \mathrm{t}_{\mathrm{i}} \mathrm{t}_{\mathrm{j}}\right\}$.

Moments: $[c] 40: 20, M R 5: 42$.

Marginal distributions, conditional distributions, regression: [4] 70 .

Independence of quadratic forms: $\quad[0] 1: 83$.

Distributions of moments, partial and multiple correlations: $\quad[i] 24: 185,[i] 27: 235,[i] 28: 20$.

$\mathrm{D}$ (product moment) $=$ Wishart: $\quad[c] 20: 32, \quad[i] 20$ : 218, [u]35:336, [u]29:260, 271, MR10:387, [b]17:82.

Cumulants of logarithmic generalized variance: [i] $38: 1 \%$.

Independence of distribution of means and second order moments: [4]233.

$\mathrm{D}$ (multiple correlation): $\quad[d] 3: 196$.

Fiducial distribution: $\quad[u] 34: 41$.

Multiple and partial correlation: $\quad[c] 19: 100$.

Linear regression theory: [4]245.

$\mathrm{D}(\mathrm{Q})=\chi^{2}: \quad[4] 104$.

$\mathrm{D}$ (various $\mathrm{Q}): \quad$ MR13:142.

$\mathrm{D}$ (vector correlation): $\quad[c] 28: 353$.

Sampling: [4]XI, [d]6:202.

$\operatorname{MLE}\left(\mathrm{m}_{1}, \ldots, \mathrm{m}_{\mathrm{n}}\right)=\left(\overline{\mathrm{x}}_{1}, \ldots, \overline{\mathrm{x}}_{\mathrm{n}}\right): \quad[6] 18 \%$.

Hotelling's generalized Student test: [4]234, $[d] 9: 240$.

LR test for variances equal and correlations zero: $[d] 11: 204$.

LR test for independence of variables: $[d] 11: 1 \%$.

Characterization: $\quad[e] 14: 36 \%$.

Hypothesis of equality of means: [4]238.

Independence of sets of variables: [4]242.

Probability that all $n$ variables are positive: $\quad[d] 26$ : 484.

Tolerance regions: $[d] 27: 174$.

Testing variance-covariance homogeneity: 31, [c] $34: 311$.

$[c] 31:$
Truncation: $[x] 5: 1 \%$.

Various tests: $\lfloor d] 17: 25 \%,[d] 21: 293$.

Quadrivariate: $[c] 43: 206$.

Bibliography of tests of hypothesis of equality of variances called 'Bipolar' distribution: $[e] 14: 61$.

Variables separated into two sets: $[c] 30: 295$.

Many samples: $[c] 31: 221$.

Seq. analysis: $\quad[e] 12: 328$.

Central limit theorem: $\quad[i] 28: 109$.

Generalizations of $\mathrm{N}(\mathrm{m}, \mathrm{v})$ theorems: $\quad[e] 17: 221$.

See also: $[d] 8: 149,[d] 17: 344,[d] 19: 447,[d] 21$ : 445, [e]3:273, [e]12:99, [e]6:35, [a]90:136, [c]6:59, $[c] 15: 192,[c] 35: 58,[0] 1: 79,[b] 18: 70,[c] 43: 212$, $[x] 1: 59,[t] 6: 181,[g] 52: 200$, MR13:366, MR17: 278, MR12:345, MR15:141, MR6:159, Z10:406, Z15:220, MR10:312, Trans. Am. Math. Soc. $24: 135$.

\section{Type III Distributions}

\subsection{Type III $(p, q)$}

$D(x)=\frac{p^{q}}{\Gamma(q)} x^{q-1} e^{-p x},(0, \infty)$

$\mathrm{D}(\mathrm{x})=\frac{\mathrm{x}^{\mathrm{b}} \mathrm{e}^{-\mathrm{x} / \mathrm{a}}}{\mathrm{b} ! \mathrm{a}^{\mathrm{b}+1}}($ "gamma"); [6]112.

$\mathrm{D}(\mathrm{x})=$ Type III (k/a, k) ("Erlang"): [ [d]24:339

$\mathrm{D}(\mathrm{x})=$ Type III (p/q, p) ("Eulerian"): [c]35:6.

$\mathrm{Ch}(\mathrm{x})=(1-\mathrm{it} / \mathrm{p})^{-\mathrm{q}}: \quad$ [2]55, [1]126.

$\operatorname{MGF}(\mathrm{x})=(1-\mathrm{at})^{-\mathrm{b}-1}: \quad[6] 115,[4] \% 4$.

$\alpha_{1}=\mathrm{q} / \mathrm{p}, \alpha_{2}=\mathrm{p}^{-2} \mathrm{q}(\mathrm{q}+1), \alpha_{3}==\mathrm{p}^{-3}(\mathrm{q}+1)(\mathrm{q}+2)$ : 55.

$\mathrm{E}(\mathrm{x})=\mathrm{a}(\mathrm{b}+1), \quad \mathrm{v}=\mathrm{a}^{2}(\mathrm{~b}+1)$

$\mathrm{r}^{\text {th }}$ cumulant $=\mathrm{q}(\mathrm{r}-1) ! \mathrm{p}^{-\mathrm{r}} \quad$ [2]6\%.

$\mu_{2}=\sigma^{2}=\mathrm{qp}^{-2}, \mu_{3}=2 \mathrm{qp}^{-3}, \mu_{4}=3 \mathrm{q}(\mathrm{q}+2) \mathrm{p}^{-4}: \quad$ [2] 433 .

Arithmetic, geometric means: Math. Student 13: 11.

$\mathrm{C}(\mathrm{x}): \quad[c] 25: 379$.

$\mathrm{D}(\mathrm{x} /(\mathrm{x}+\mathrm{y}))=$ Beta : $\quad[14] 41$.

$\sim \mathrm{C}(\mathrm{x}): \quad$ MR13:553.

Transformations $\mathrm{y}=\mathrm{x}^{\mathrm{n}}, \mathrm{y}=\mathrm{e}^{\mathrm{x}}: \quad[d] 9: 176$.

Normalizing transform: Proc. Pak. Stat. Assoc. 5:120.

Transformation $\mathrm{y}=(\mathrm{x}+\mathrm{k})^{\frac{1}{2}}: \quad[d] 14: 115$.

Moments, Ch(x), cumulants when $\mathrm{x}=\mathrm{y}-\mathrm{c}$ : $1-136,1-144$.

Type III $(\mathrm{p}, \mathrm{p}+1): \quad[e] 5: 176$.

$\mathrm{D}(\overline{\mathrm{x}})=$ Type III (np, nq): [2]244, $[c] 18: 335,[w]$ $1: 73$.

$\mathrm{D}\left[(\mathrm{nq}-1) \mathrm{n}^{-1} \overline{\mathrm{x}}^{-1}\right]=$ Reciprocal Type $\mathrm{III}(\mathrm{p}, \mathrm{nq}-1)$ : $\sim \mathrm{D}(\sqrt[3]{\mathrm{x}}): \quad[c] 35: 297$. 
$\mathrm{D}(\mathrm{x} / \mathrm{y})=$ Beta of second kind $(\mathrm{p}=\mathrm{q}): \quad[w] 1: 74$.

$\mathrm{FD}\left(\mathrm{p}^{-1}\right)=$ Type $\mathrm{V}(\mathrm{n} \overline{\mathrm{x}} / \mathrm{nq}-1, \mathrm{nq}-1): \quad[3] 87,[c]$ 30:408.

Bayes $\mathrm{D}\left(\mathrm{p}^{-1}\right)=$ Rectangular: [3]91.

$\mathrm{D}(\mathrm{xy})$ where $\mathrm{x}$ is Type III and $\mathrm{y}$ is Type $\mathrm{V}$ : [i] $39: 64$.

$\mathrm{D}(\mathrm{x}-\mathrm{y})=$ No. 8.92 , where $\mathrm{D}(\mathrm{x})=$ Type III $(\lambda, \mathrm{N})$ and $\mathrm{D}(\mathrm{y})=$ Type III $(\mu, 1)$

$\mathrm{D}(\mathrm{HM}): \quad$ MR4:164.

D (xy), Ch(xy): MR16:37\%.

$\mathrm{D}(\mathrm{xy})=$ Bessel, $\mathrm{D}(\mathrm{xyz}): \quad$ Studies Presented to Richard von Mises, p. 301, Acad. Press, 1954.

MLE $(p)=$ Moment estimate $(p)=q / \bar{x}$, correcting for bias $=(n q-1) / n \bar{x}$, sufficient,$\sim$ efficient, not efficient : [3]26.

$\operatorname{Var}((n q-1) / n \bar{x})=p^{2}(n q-2)^{-1}:$

$[1] 505,[u] 45: 214$.

$\operatorname{MLE}(1 / \mathrm{p})=\overline{\mathrm{x}} / \mathrm{q}: \quad[3] 21$.

$\operatorname{Var}(\overline{\mathrm{x}} / \mathrm{q})=\mathrm{p}^{-2} \mathrm{n}^{-1} \mathrm{q}^{-1}$, sufficient: [3]21.

Sufficient statistics for $\mathrm{p}: \quad[e] 17: 212,219$.

Ordered LSE is MLE for 1/p: [d]25:315.

MLE (p,q), variance-covariance of estimates: 14:187, $[c]$ 42:22, $[r] 1: 18$.

$\operatorname{UMVUE}(1 / \mathrm{p})=\overline{\mathrm{x}} / \mathrm{q}$, with $\operatorname{Var}=\mathrm{p}^{-2} \mathrm{n}^{-1} \mathrm{q}^{-1}$ :

[3]53.

There is a sufficient estimate of $\mathrm{q}$ : [3]26.

Estimation: $[e] 8: 324$.

Minimax: $[16] 64,[e] 14: 5 \%$.

Gauging: $\quad[e] 15: 192$.

Closest estimate: $[u] 33: 21 \%$.

Testing n such populations: [3]325.

Slippage tests for p: Koninkl. Nederl. Akad. (A) 59:329.

Testing equality of 1/p: $\quad[c] 31: 205$.

Confidence intervals for $1 / \mathrm{p}:[3] \% 4,[e] 6: 113$, MR5:128.

Truncated distribution: $[g] 45: 411$.

Estimation from truncated Type III: [d]26:659, $[d] 27: 4.98$.

Truncated samples: $\quad[c] 40: 52$.

D (gap between events $)=$ generalized Poisson: 3:123, [o]11:101.

Characterized by independence of sum and quotient: $[d] 26: 319$.

Discrete Type III: $[c] 44: 365$.

Mills ratio: $\quad[d] 24: 309$.

Normal limit: Am. Math. Monthly 50:98.

Renewal theory: $[d] 11: 448$.

Trivariate: $[d] 21: 550$.

See also: $[d] 7: 95,[d] 8: 17,[d] 24: 40 \%,[e] 2: 150$, $[b] 11: 101,[c] 21: 263,[d] 25: 640,[c] 24: 300,[v] 2: 330$, [g]50:904, [i] 39:171, [q]7:95, Am. Math. Monthly $50: 98, M R 17: 756$.

\subsection{Type III $(\mathrm{p}, 1)$}

$\mathrm{D}(\mathrm{x})=\mathrm{pe}^{-\mathrm{px}}$, "negative exponential"

$[5] 34$.

Туре X.

$\alpha_{1}=1 / \mathrm{p}: \quad[5] 59,[2] 48$.

$\mathrm{v}=\mathrm{p}^{-2}: \quad[5] 6 \%$.

Moments: [2]1 42, [8]100, [10]18.

Characterization: $\quad[t] \%: 60$, 3d Berkeley Symp. 2: 195.

Cumulants: [2]8\%, [10]40.

$\mathrm{C}(\mathrm{x}): \quad[c] 25: 379$.

Mean difference: $[c] 28: 432$.

$\operatorname{MGF}(\mathrm{x})=(1-\mathrm{t} / \mathrm{p})^{-1}: \quad[10] 3 \%$.

$\operatorname{MGF}(\log \mathrm{x}): \quad[v] \%: 296$.

Grouping corrections: $\quad[c] 39: 433$.

A priori distributions of p: $[i] 27: 36$.

$\mathrm{D}(\mathrm{x}+\mathrm{y}): \quad[8] 95$.

If $\mathrm{D}(\mathrm{x})=\mathrm{pe}^{-\mathrm{px}}, \mathrm{D}(\mathrm{y})=\mathrm{Pe}^{-\mathrm{Py}}$,

$$
\text { then } \mathrm{D}(\mathrm{x}-\mathrm{y})=\begin{aligned}
& A \mathrm{e}^{-\mathrm{p}(\mathrm{x}-\mathrm{y})}, \mathrm{x}>\mathrm{y} \\
& A \mathrm{e}^{\mathrm{P}(\mathrm{x}-\mathrm{y})}, \mathrm{x}<\mathrm{y}
\end{aligned}
$$

where $\mathrm{A}(\mathrm{p}+\mathrm{P})=\mathrm{pP}$ :

Examples and applications: $\quad$ [8]29,79,83, $[j] 20: 366$, [c]39:168.

$\mathrm{D}(\overline{\mathrm{x}}): \quad[c] 39: 168$.

$\mathrm{D}(\xi)=\operatorname{No} .8 .9, \operatorname{MGF}(\xi): \quad[p]^{\gamma}: 153$.

$\Sigma \mathrm{x}_{1}$ where $\mathrm{x}_{1}$ is Type $\operatorname{III}\left(\mathrm{p}_{1}, 1\right): \quad$ MR5:42.

Rank variates: $\quad[c] 24: 210,271,[r]]_{4}: 153$.

Estimation: $[c] 35: 18 \%,[g] 48: 493,[s] 10: 167,[0] 8$ : $15,[p]^{7}: 152$.

Censored sample: $\quad[c] 41: 230,[d] 23: 23 \%$.

Moments of $\mathrm{D}(\mathrm{s}): \quad[c] 22: 53$.

Variance of mean deviation $\cong 4 / 3 \mathrm{np}^{2}:$ [2]21\%.

Testing $\mathrm{p}=\mathrm{p}_{0}: \quad[d] 9: 84$.

Sequential test: $\quad[c] 41: 252,[d] 27: 460$.

Testing against four other possible dist:

Confidence intervals: $\quad[3] 84$.

Estimation from truncated exponential: $\quad[d] 26: 4.98$

Relation with Poisson, D. L. Gerlough and A. Schuhl, Poisson and Traffic, The ENO Foundation, 1955: [o]2:13.

See also: $\quad[d] 7: 19,[d] 25: 555,[g] 48: 488,[g] 50: 904$.

If $\mathrm{x}=\mathrm{y}-\mathrm{c}$, Mean $=\mathrm{c}+\frac{1}{\mathrm{p}}$, $\operatorname{var}=\frac{1}{\mathrm{p}^{2}}$, skewness $=2$, kurtosis $=6, \operatorname{Ch}(\mathrm{x})$, cumulants: $\quad[18] 1-136,1-144$.

MLE: $\quad[k] 8: 52$. 
LR test for hypothesis that $\mathrm{n}$ such populations are identical, etc: [3]305.

k samples: Z14:269.

Best linear estimates of $\mathrm{m}$ and $\sigma: \quad[d] 25: 320$.

$\mathrm{D}\left\{\frac{1}{\mathrm{n}-1} \sum_{i=1}^{\mathrm{n}-1}\left|\mathrm{x}_{\mathrm{i}+1}-\mathrm{x}_{1}\right|\right\}: \quad[v] 6: 133$.

Life testing: $\quad[d] 25: 373$.

Original Neyman-Pearson paper on hypothesis testing: $\quad[c] 20: 221$.

Censored samples: $[g] 52: 58$.

Estimation by order statistics: $\quad[d] 26: 585$.

Quasi-range: $\quad[d] 28: 179$.

LR tests: [d]12:301.

Seq. testing: $\quad[x] 2: 86$.

Confidence intervals: $\quad M R 5: 43$.

$\left\{\begin{array}{r}H: p=p_{0}, c=c_{0} \\ \text { Alt: } p=p_{1}, c=c_{1}\end{array}\right\} \quad[3] 304$.

See also: $[d] 25: 409,[d] 24: 458,[c] 30: 402,416$, $[e] 24: 279$.

Maxwell-Bolzmann.

$\mathrm{x}^{2}$ is Type III $(\mathrm{p}, 3 / 2): \quad[5] 39,60,[s] 1: 304$.

Connection with $\mathrm{N}(0, \mathrm{v}): \quad$ [12]40.

$\mathrm{D}(\overline{\mathrm{x}}): \quad[n] 10-3: 90$.

See also: $\quad[n] 1 \%-1: 125$.

\subsection{Type III $(1, \mathrm{q})$}

$\mathrm{D}(\mathrm{x})=\frac{1}{\Gamma(\mathrm{q})} \mathrm{e}^{-\mathrm{x}} \mathrm{x}^{\mathrm{q}-1}$, "Gamma": $\quad[10] 149$.

$\alpha_{\mathrm{r}}=\Gamma(\mathrm{q}+\mathrm{r}) / \Gamma(\mathrm{q}): \quad[10] 150,163$.

$\mathrm{k}_{\mathrm{r}}=\mathrm{q}(\mathrm{r}-1)$ !: [2]96,153.

$\mathrm{Ch}(\mathrm{x})=(1-\mathrm{it})^{-\mathrm{q}}: \quad[17]$ No. 34.

Skewness $=\mathrm{q}^{-\frac{1}{2}}: \quad[10] 161$.

$\mathrm{HM}=\mathrm{q}-1: \quad[10] 163$.

$\mathrm{D}(\mathrm{n} \overline{\mathrm{x}})=$ Type III $(1, \mathrm{nq}): \quad[c] 19: 228,[n] 10-3: 91$.

$\mathrm{D}\left(\Pi \mathrm{x}_{\mathrm{i}}\right): \quad[c] 24: 4 \% 4$.

$\mathrm{D}\left(\mathrm{x}_{1}-\mathrm{x}_{2}\right): \quad$ [2]252, [c]24:293.

$\mathrm{D}\left(\mathrm{x}_{1}+\mathrm{x}_{2}\right)=$ Type III $\left(1, \mathrm{q}_{1}+\mathrm{q}_{2}\right): \quad[10] 151,[p]^{7}: 101$.

$\mathrm{D}(\mathrm{GM})$ as a series: [2]251.

With generalization: $[d] 5: 27 \%$.

$\mathrm{D}\left[\mathrm{x}_{1} /\left(\mathrm{x}_{1}+\mathrm{x}_{2}\right)\right]=\mathrm{B}\left(\frac{1}{2} \mathrm{q}_{1}, \frac{1}{2} \mathrm{q}_{2}\right): \quad[10] 153$.

$\mathrm{D}\left(\mathrm{x}_{1} / \mathrm{x}_{2}\right)=$ Beta of second kind: $\quad[10] 158,160,[p]^{7}$ : 102.
$\mathrm{D}\left(\mathrm{x}_{1}-\mathrm{x}_{2}\right)$ involves a Bessel function if $\mathrm{x}_{1}$ and $\mathrm{x}_{2}$ are from two separate distributions and $\mathrm{D}\left(\mathrm{x}_{1} / \mathrm{x}_{2}\right)$ is Fisher: $\quad[d] \%: 51$.

$\sim \mathrm{D}(\overline{\mathrm{x}}): \quad[d] 25: 636$.

$\mathrm{D}(\overline{\mathrm{x}}, \mathrm{GM} / \overline{\mathrm{x}})=\mathrm{D}(\overline{\mathrm{x}}) \mathrm{D}(\mathrm{GM} / \overline{\mathrm{x}}): \quad[c] 30: 287$.

C. - R. $(q)=\left[n\left(d^{2} / d q^{2}\right) \log \Gamma(q)\right]^{-1}$

$\overline{\mathrm{X}}$ is moments estimate of $\mathrm{q}$, not sufficient.

Closest estimate: $[u] 33: 216$.

$\operatorname{MLE}(q)=\log$ GM: $\quad[p]^{\gamma} \%: 169$.

$\mathrm{E}(\overline{\mathrm{x}})=\mathrm{q}, \operatorname{var}(\overline{\mathrm{x}})=\mathrm{q} / \mathrm{n}$, efficiency $(\overline{\mathrm{x}}) \rightarrow 0: \quad[1] 504,5$.

$\sim \mathrm{D}(\log \mathrm{GM})=$ Normal: $\quad$ [1]505.

Confidence intervals: $[p]^{7}: 224$.

Mellin transform: $\quad[d] 19: 373$.

$\log \log (1 / \mathrm{x}): \quad[v] 8: 71$.

$\mathrm{A}+\mathrm{B} \log \mathrm{x}: \quad[c] 36: 165$.

Multivariate generalization: $\quad[d] 22: 549$.

Tetrachoric functions: $[c] 14: 161$.

Fermi-Dirac, $\mathrm{x}-\mathrm{c}$ is Type III $(3 / 2$,const.): 42, $[j] 8: 701,[s] 1: 304$.

See also: $\quad[d] 25: 401,[10] 161,[d] 22: 425,[c] 24: 281$, $[c] 27: 409,[c] 30: 415,[c] 36: 165,[d] 25: 784,[e] 10: 314,[g]$ $51: 467,[c] 44: 265,[d] 22: 418$, Can. J. Math. 3:140.

\subsection{Type III $(1,1)$}

$\mathrm{D}(\mathrm{x})=\mathrm{e}^{-\mathrm{x}}$, "exponential": $\quad[6] 21 \%$.

$\mathrm{Ch}(\mathrm{x})=1 /(1-\mathrm{it}): \quad[1 \mathrm{y}]$ No. 33 .

$\mathrm{D}(\overline{\mathrm{x}}, \mathrm{s}): \quad[d] 3: 128,[d] 4: 133,[d] 4: 139,142$.

$\mathrm{x}=\mathrm{y}-\mathrm{c}$, Type $\mathrm{X}$, confidence intervals: $[b] 17: 90$.

$\mathrm{D}\left(\Sigma \mathrm{x}_{1}\right)=$ Type III by convolutions: $\quad[d] 5: 13$.

$\mathrm{D}\left(\Sigma \mathrm{x}_{1} / \mathrm{i}\right)=\mathrm{D}\left(\max \mathrm{x}_{\mathrm{i}}\right): \quad[b] 14: 43$.

Doubly truncated, Ch(x): $\quad[n] 10: 3,[17]$ No. 32.

Ratio of two ranges: $[d] 21: 112$.

C. - R. theorem false for $\mathrm{x}=\mathrm{y}-\mathrm{c}: \quad[1] 485,[3] 4 \%$.

See also: $\quad[d] 22: 425,[i] 36: 152,[m] 6: 120,[d] 22:$ 418.

\subsection{Chi-Square $(\mathbf{k})$}

$\mathrm{D}(\mathrm{x})=\left[1 / 2^{\frac{1}{2} \mathrm{k}} \Gamma\left(\frac{1}{2} \mathrm{k}\right)\right] \mathrm{x}^{\frac{1}{2} \mathrm{k}-1} \mathrm{e}^{-\frac{1}{2} \mathrm{x}}: \quad[5] 96,[10] 164,[2] 17$, [4]102, [8]134, MR8:161, [1]3:353, [18]1-161.

Type $\operatorname{III}\left(\frac{1}{2}, \frac{1}{2} \mathrm{k}\right)$ :

$\alpha_{1}=\mathrm{k}, \mathrm{v}=2 \mathrm{k}: \quad[1] 234,[4] 103$.

$\alpha_{\mathrm{s}}=\mathrm{k}(\mathrm{k}+2) \ldots(\mathrm{k}+2 \mathrm{~s}-2): \quad$ [1]234.

$\mu_{2}=2 \mathrm{k}, \quad \mu_{3}=8 \mathrm{k}, \quad \mu_{4}=48 \mathrm{k}+12 \mathrm{k}^{2}, \quad \mu_{5}=32 \mathrm{k}(5 \mathrm{k}+12)$ : [2]292.

Cumulants: $\quad[c] 31: 216$.

$\mathrm{Ch}(\mathrm{x})=(1-2 \mathrm{it})^{-\frac{1}{2} \mathrm{k}}: \quad[v]_{4}: 8$.

$\mathrm{C}(\mathrm{x})$, relation with Poisson: $[l] 3: 35 \%,[c] 3 \%: 313$. 
Introduction, properties, examples: [15]253.

Obtained as dist. of normal variance: [3]104, Z23: $148,[p]^{7}: 98$.

$\mathrm{D}\left(\mathrm{x}_{1}+\mathrm{x}_{2}\right): \quad[e] \gamma: 27$.

$\mathrm{D}(\log \mathrm{x}): \quad[c] 34: 170$.

$\mathrm{D}(2 \sqrt{\mathrm{xy}})=$ Chi square $(2 \mathrm{n}-2)$ if $\mathrm{D}(\mathrm{x})=$ chi-square $(\mathrm{n})$ and $\mathrm{D}(\mathrm{y})=$ chi-square $(\mathrm{n}-1)$.

$\mathrm{D}(\overline{\mathrm{x}}, \mathrm{s})$ for $\mathrm{k}=2,3,4, \quad \mathrm{n}=3,4: \quad$ MR12:345.

$\mathrm{D}\left(\mathrm{x}_{1} / \mathrm{x}_{2}\right)=$ Beta of second kind $\left(\frac{1}{2} \mathrm{k}_{1}, \frac{1}{2} \mathrm{k}_{2}\right): \quad[10] 17 \%$.

$\mathrm{D}\left[\mathrm{x}_{1} /\left(\mathrm{x}_{1}+\mathrm{x}_{2}\right)\right]=\operatorname{Beta}\left(\mathrm{k}_{1}, \mathrm{k}_{2}\right): \quad[10] 17 \%$.

Obtained as $\mathrm{D}\left(\Sigma \mathrm{x}_{\mathrm{i}}^{2}\right)$ : $\quad[10] 169$.

$\sim \mathrm{D}\left[(\mathrm{x}-\mathrm{k})(2 \mathrm{k})^{-\frac{1}{2}}\right]=\mathrm{N}\left[\mathrm{k},(2 \mathrm{k})^{-\frac{1}{2}}\right], \sim \mathrm{D}(2 \mathrm{x})^{\frac{1}{2}}$

$=\mathrm{N}\left[(2 \mathrm{k})^{\frac{1}{2}}, 1\right]: \quad[1] 251$.

$\sim \mathrm{D}(-2 \log \mathrm{LR}): \quad[d] 9: 60$.

Reproductive property: [4]105, [10]17\%.

Normal approximation: $[d] 17: 216,[d] 27: 786$.

Large parameter: $\quad[c] 43: 92$, Proc. A.M.S. 6th Symp. in Appl. Math., p. 251.

Convolution for a pair of Chi-square variables: 134.

$[8]$

Percentage points: $[c] 41: 313,[i] 33: 168$.

$\sim$ Significance levels: $\quad[d] 14: 57,93$.

Elderton's tables: $\quad[c] 1: 155$.

Minimax estimation: $\quad[16] 1 \%$.

Connection between $\chi^{2}$ test and $\chi^{2}$ distribution: $[c] 19: 215$.

Original Neyman-Pearson paper on hypothesis testing: $\quad[c] 20: 263$.

Queueing: $\quad[b] 16: 82$

Approximation for small sample: $[d] 9: 158$.

See also: $[d] 18: 89,[g] 29: 372,[c] 22: 298,[a] 85:$ $87,95,[a] 87: 442,[c] 29: 133,[c] 29: 389,[c] 31: 346,[c] 34:$ $368,[c] 32: 268,[c] 40: 421,[p]^{7}: 98$.

\subsection{Non-Central Chi-Square}

$\mathrm{D}(\mathrm{x})=\mathrm{e}^{-\frac{1}{2} \mathrm{x}} \mathrm{e}^{-\frac{1}{2} \mathrm{k}} 2^{-\frac{1}{2} \mathrm{n}} \sum_{\mathrm{j}=0}^{\infty} \frac{\mathrm{x}^{\frac{1}{2} \mathrm{n}+\mathrm{j}-1} \mathrm{k}^{\mathrm{j}}}{\Gamma\left(\frac{1}{2} \mathrm{n}+\mathrm{j}\right) 2^{2 \mathrm{j}} \mathrm{j} !} \quad[c] 36: 204$.

$\mathrm{Ch}(\mathrm{x})=\frac{\exp [\mathrm{kit} /(1-2 \mathrm{it})]}{(1-2 \mathrm{it})^{\mathrm{n} / 2}}$,

$$
\mathrm{k}_{\mathrm{r}}=2^{\mathrm{r}-1}(\mathrm{r}-1) !(\mathrm{n}+\mathrm{rk}), \sim \text { form : } \quad[18] 1-162 .
$$

Logarithmic noncentral Chi-square: $\quad[0] \%: 5 \%$.

See also: $[i] 34: 57,[c] 41: 538,[i] 36$ (supplement): $18,[d] 28: 678[c] 44: 528$.

\subsection{Helmert (p, q)}

$\mathrm{D}(\mathrm{x})=\frac{(\mathrm{x} / \mathrm{q})^{\mathrm{p}-1} \mathrm{e}^{-\frac{1}{2} \mathrm{x}^{2} / \mathrm{q} 2}}{\mathrm{q} \Gamma\left(\frac{1}{2} \mathrm{p}\right) 2^{\frac{1}{2}(\mathrm{p}-2)}}: \quad[5] 94$
For $2 q^{2}=k, \quad p=2$, called Rayleigh:

[12]39.

$\mathrm{D}\left(\mathrm{x}^{2} / \mathrm{q}^{2}\right)=$ Chi-square

Called seminormal: [i]20:61.

Refs, $\operatorname{Ch}(\mathrm{x}): \quad[17]$ No. 42.

$\alpha_{1}=\frac{\left[\frac{1}{2}(\mathrm{p}-1)\right] !}{\left[\frac{1}{2}(\mathrm{p}-2)\right] !} 2^{\frac{1}{2}} \mathrm{q}, \mathrm{v}=\mathrm{pq}^{2}-\alpha_{1}^{2}$

Non-central: $\quad[d] 23: 467$.

See also: $\quad[c] 23: 418$, Elec. Eng., Nov. 1954, p. 1004, MR8:161, J. Appl. Phys. 23:137.

\subsection{Reciprocal Type III (p, q)}

$\mathrm{D}(\mathrm{x})=\frac{\mathrm{p}^{\mathrm{q}+1} \mathrm{q}^{\mathrm{q}+1}}{\Gamma(q+1)} \mathrm{x}^{-\mathrm{q}-2} \mathrm{e}^{-\mathrm{pq} / \mathrm{x}}, \mathrm{pq}>0$,

Mean $=p$, var $=\frac{p^{2}}{q-1}$, Type $V$,

$\alpha_{\mathrm{r}}=(\mathrm{pq})^{\mathrm{r}} \Gamma(\mathrm{q}-\mathrm{r}+1) / \Gamma(\mathrm{q}+1): \quad$ [2]86, 142.

Obtained from Pearson equation: [11]/45.

Various constants, with an example: [11]\%8, $[d] 3: 20$.

$\mathrm{Ch}(\mathrm{x})$ in special case: $[17]$ No. $4 \%$.

If $\mathrm{q}=-\frac{1}{2}, \quad \mathrm{p}=-1, \mathrm{Ch}(\mathrm{x})=\exp \quad[(-1+\mathrm{i}) \quad \sqrt{\mathrm{t}}]$ : $[s] 208: 318,[17]$ No. 49.

More generally: $\quad[d] 7 \%: 25$

MLE: $\quad[r] 1: 19$.

As distribution of precision constant in normal sample: $\quad[a] 97: 132$.

$\mathrm{C}(\mathrm{x}): \quad[c] 25: 379$.

See also: $\quad[c] 26: 388,[c] 36: 165,[0] 8: 55$.

\subsection{Generalized Type III}

$\mathrm{D}(\mathrm{x})=\mathrm{C}(1+\mathrm{x} / \mathrm{a})^{\mathrm{pa}} \mathrm{e}^{-\mathrm{px}}$, with moments of $\mathrm{D}(\mathrm{s})$ : $[c] 22: 52$.

$\mathrm{D}(\mathrm{x})=\left[\mathrm{p}^{\mathrm{p}} \mathrm{e}^{-\mathrm{p}} / \mathrm{q}^{\mathrm{p}} \Gamma(\mathrm{p})\right](\mathrm{q}+\mathrm{x})^{\mathrm{p}-1} \mathrm{e}^{-\mathrm{px} / \mathrm{q}}$, with semi-invariants, D ( $\bar{x})$ etc: $\quad[c] 21: 287,[c] 24: 293$.

$\mathrm{Ch}(\mathrm{x})=\mathrm{e}^{-\mathrm{aix}}[1-(\mathrm{ix} / \mathrm{p})]^{-\mathrm{ap}-1}: \quad[17]$ No. 35 .

$\mathrm{D}(\mathrm{x})=[1 / \mathrm{p} \Gamma(\mathrm{q})][(\mathrm{x}-\mathrm{c}) / \mathrm{p}]^{\mathrm{q}-1} \exp [(\mathrm{c}-\mathrm{x}) / \mathrm{p}], \mathrm{c} \leq \mathrm{x}<\infty$.

$\operatorname{MLE}(\mathrm{c}, \mathrm{p}, \mathrm{q}): \quad[3] 39$

Variance of estimates: $\quad[3149$.

Tables: $[d] 1: 191$.

Estimation: $[g] 48: 336$.

$\mathrm{D}(\mathrm{x})=\left[\mathrm{e}^{-\mathrm{ba}} \mathrm{b}^{\mathrm{p}} / \Gamma(\mathrm{p})\right] \mathrm{e}^{-\mathrm{bx}}(\mathrm{x}+\mathrm{a})^{\mathrm{p}-1}$ from Pearson's equation: $\quad[4]^{7} 4,[2] 124,[11] 65$,

$\mathrm{D}(\overline{\mathrm{x}}): \quad[n] 10-3: 91$.

$\mathrm{D}(\mathrm{x})=\mathrm{A}(\mathrm{x}-\mathrm{c})^{\mathrm{q}-1} \mathrm{e}^{-\mathrm{p}(\mathrm{x}-\mathrm{c})}, \quad \mathrm{x}>\mathrm{c}, \quad \mathrm{p}>0, \quad \mathrm{q}>0$. [1]249. 
Various constants, with an example: [11]66.

One root of quadratic in Pearson equation is $\infty$ : $[11] 44$.

$\mathrm{D}\left(\mathrm{x}_{1} / \mathrm{x}_{2}\right)$ where each is Generalized Type III, or one is Generalized Type III and the other $\mathrm{N}(\mathrm{m}, \mathrm{v})$ : [2]253.

Bayes' Theorem: $[n] 16-1: 114$.

Counting radioactive particles: $\quad[d] 18: 260$.

Two Generalized Type III distributions added: $[n] 8-3: 76$.

See also: $[d] 1: 150,[d] 1: 191,[d] 7: 18,[c] 1: 293$, $[c] 3: 311,[c] 5: 173,[c] 13: 13,[c] 16: 114,[n] 1-3: 88,[c] 32:$ $294,[u] 46: 284$.

\subsection{Wishart Univariate}

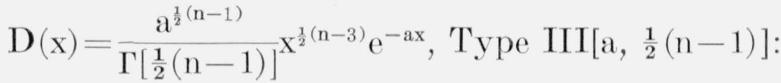

[1] 391 .

\section{Binomial Distributions}

\subsection{Binomial $(\mathrm{k}, \mathrm{p})$}

$\mathrm{D}(\mathrm{x})=\left(\begin{array}{l}\mathrm{k} \\ \mathrm{x}\end{array}\right) \mathrm{p}^{\mathrm{x}}(1-\mathrm{p})^{\mathrm{k}-\mathrm{x}}, \mathrm{x}=0,1, \ldots, \mathrm{k}$, "Bernoulli": $[4] 4 \%,[10] 46,[5] 106,[d] 1: 118$.

$\alpha_{1}=\mathrm{kp}, \quad \mathrm{v}=\mathrm{kpq}, \quad($ where $\mathrm{p}+\mathrm{q}=1), \alpha_{3}=\mathrm{kpq}(\mathrm{q}-\mathrm{p})$, $\alpha_{4}=2 \mathrm{k}^{2} \mathrm{p}^{2} \mathrm{q}^{2}+\mathrm{pq}(1-6 \mathrm{pq})$ :

$[5] 57,66,[10] 58,[2] 52,117$.

$\mu_{\mathrm{r}+1}=\mathrm{pq}\left(\mathrm{kr} \mu_{\mathrm{r}-1}+\mathrm{d} \mu_{\mathrm{r}} / \mathrm{dp}\right): \quad$ [2]118

$\beta_{1}=(\mathrm{q}-\mathrm{p}) / \sqrt{\mathrm{kpq}}, \quad \beta_{2}=3+(1-6 \mathrm{pq}) / \mathrm{kpq}$.

Factorial moments $\alpha_{[i]}=\mathrm{K}^{[1]} \mathrm{p}^{1}$, where $\mathrm{k}^{[i]}=\mathrm{k}(\mathrm{k}-1)$ ... $(\mathrm{k}-\mathrm{i}+1)$ : [1]25\%, Nature 164:282, [2]8\%.

Long introductory article: [15]23.

Moments: $\quad[d] 6: 96,[v] 3: 325,29: 28$.

Cumulants $\quad \kappa_{\mathrm{r}+1}=\mathrm{pq}\left(\mathrm{d} \kappa_{\mathrm{r}} / \mathrm{dp}\right): \quad$ [2]135, [c]31:392 [18]1-144, [d]2:196.

Mean deviation: $\quad[c] 44: 532, M R^{7}: 128$.

Several formulas for moments about the mean: $[d] 7: 191,[c] 15: 410, Z 9: 220$.

$\mathrm{C}(\mathrm{x}): \quad[c] 38: 423$.

$\mathrm{C}(\mathrm{x})$ as a Beta integral: $\quad[6] 235,[18] 1-152$.

Recursion formula for moments: [14]33.

Moments in general: $\quad[d] 8: 103,[d] 11: 106,[c] 1 \%: 165$ [c]26:262, MR7:461, [c]30:11, Bull. Am. Math. Soc. 40:262, $41: 85 \%$.

Moments and series: $\quad[i] 14: 168$.

Aresin transform: [14]210.

Another form of $\mathrm{D}(\mathrm{x}): \quad[d] 8: 116$.

$\beta_{1}=(\mathrm{q}-\mathrm{p})^{2} / \mathrm{kpq}, \quad \beta_{2}=[1+3 \mathrm{pq}(\mathrm{k}-2)] / \mathrm{kpq}$, etc. : $[12] 52,[d] 4: 216$. $\mathrm{kp}-\mathrm{q} \leq \operatorname{mode} \leq \mathrm{kp}+\mathrm{p}: \quad[6] 5 \%$.

$\mathrm{Ch}(\mathrm{x})=\left(\mathrm{pe}^{\mathrm{it}}+1-\mathrm{p}\right)^{\mathrm{k}}: \quad$ [5]62, [2]55,103.

$\operatorname{MGF}(\mathrm{x})=\left(\mathrm{q}+\mathrm{pe}^{\mathrm{t}}\right)^{\mathrm{k}}: \quad$ [4] $48,[10] 38$.

$\mathrm{PGF}=(\mathrm{q}+\mathrm{pt})^{\mathrm{k}}: \quad[18] 1-146$.

$\sim \mathrm{C}(\mathrm{x}): \quad[j] 8: 99$.

$\mathrm{E}(1 / \mathrm{x}): \quad[g] 49: 169$.

$\mathrm{D}(\mathrm{n} \overline{\mathrm{x}})=$ Binomial $(\mathrm{nk}, \mathrm{p}): \quad$ [2]243.

$\mathrm{D}\left(\mathrm{s}^{2}\right): \quad[c] 44: 262$.

$\mathrm{FD}(\mathrm{p}): \quad[c] 3 \%: 11 \%$.

$\mathrm{D}(\mathrm{x}-\mathrm{y})$ in terms of Legendre functions: MR14:566.

Reproductive property by convolutions: [\%]216.

A convolution with respect to $\mathrm{p}: \quad[w] 6: 165$.

C. $-\mathrm{R} .(\mathrm{p})=\mathrm{pq} / \mathrm{kn}: \quad[5] 141,[15] 20 \%,[p]^{7}: 160$.

$\overline{\mathrm{x}}$ is sufficient: $\quad[p] \%: 162$.

$(\mathrm{kn})^{-1} \Sigma_{\mathrm{x}_{1}}$ is efficient and unbiased: [5]141, [1] $48 \%$.

Is MLE: [5]14.

$\sim \mathrm{BCR}$ for $\mathrm{k}=\mathrm{k}_{0}: \quad[d] 18: 556$.

Minimax and Bayes: $[d] 23: 404$.

Minimax estimation: $\quad[16] 18,[d] 21: 180$.

Modified Bayes: MR11:42.

BANE(p): $\quad[d] 21: 402$.

Biased and unbiased statistics: $[t] 5: 149$.

$\operatorname{MLE}(k, p): \quad[k] 18: 11 \%$.

Estimation of $\mathrm{p}$ based on runs: MR14:1102.

LR comparison of two binomials: $\quad[c] 3 \%: 140$.

Confidence intervals: $[w] 2: 171,[c] 41: 275,[c] 33:$ 181, [w] $: 94,[d] 9: 174,[y] 21: 17,[4] 129,[w] 19: 130$, $[w] 2: 171,[c] 41: 308$.

Tables: $[3] 81,[c] 44: 436,[0] 8: 85$.

Sequential: $\quad[d] 17: 288, \quad 489,[d] 18: 131,[b] 8: 98$, $[c] 41: 252$.

Acceptance inspection: [b]12:301, MR15:727.

Chi-square test: $\quad[k] \%: 20 \%$.

UMP test: $\quad[c] 43: 465$.

Sampling inspection: $[j] 8: 626$.

Multiple sampling: $\quad[d] 14: 363$.

Analysis of variance: $[d] 11: 335$.

Order statisties: $\quad$ MR16:729, $[s] 8: 62$.

Approximate formulas: [8]172, [d]19:592, $[n] 18$ No. 1-2:123.

Normal approximation: $[9] 131,[d] 16: 319,[c] 4$ : 190, [c]29:402, [r]4:47, Proc. Koninkl. Nederl. Akad. (A) 57:513, MR10:131.

Using generating functions: Proc. 5th Intl. Cong. Math 2:441, [w]1:41.

Inequalities for tails: [7]126.

Asymptotic behavior: $\quad$ MR15:138. 
Convergent sequences of binomials: Monthly, 50:96.

n binomials: $[e] 8: 11$.

Normalizing transform $\mathrm{y}=\mathrm{k}^{\frac{1}{2}} \sin ^{-1}(\mathrm{x}+\mathrm{a} / \mathrm{k})$ and other transforms: $[d] 14: 116,[f] 3: 52,[c] 35: 248$.

$\log \mathrm{x} /(1-\mathrm{x}),-2 \tanh ^{-1} \mathrm{x}: \quad[v] 8: 73$.

Other transforms: $[x] 2: 94$.

Choosing between several binomials: $[j] 36: 53 \%$.

If p not constant (cf. "Lexian"): [k]16:1.

Transformations, approximations, applications: [15]668.

Chain binomials: $[c] 40: 2 \% 9$.

Gambler's ruin: [i]24:52.

Connection with Beta distribution: $[c]$ 41:304, $[n] 18: 121$.

Actuarial application: [i]35:11.

Binomials added: $\quad M R 1 \%: 862,[w] 5: 73$.

Generalization: [d]20:311.

Generalized binomial

$$
\mathrm{Ch}(\mathrm{x})=\Pi\left(\mathrm{q}_{1}+\mathrm{p}_{1}{ }^{\mathrm{eix}}\right): \quad \text { [2]122. }
$$

A modification: [e]15:237, 251 .

See also: $\quad[d] 6: 27,[d] 17: 13,[d] 21: 247,[d] 22: 129$, $[e] 12: 248,[b] 14: 115,[j] 8: 364,[c] 11: 269,[c] 16: 165,202$, [f]12:276, [f]13:225, [r]1:15, 32, Bull. Am. Math. Soc. 1935, p. 857, 11th Skand. Math-Kongress p. 210,
Z5:212, Z18:31, MR6:234, MR11:604, MR12:509, MR9:450, Z3:18, [w]1:9, [g]33:390, [a]83:247, [c]33: 222, $[g] 49: 169,[i] 7: 153,[i] 6: 7 \%,[i] 20: 47,[i] 22: 23$, $[i] 26: 22,[i] 31: 8,[i] 32: 188,[d] 25: 770,[c] 44: 364, J$. Proc. Roy. Soc. N.S.W. 81:38, Z19:316, Proc. Intl. Cong. Math. (1924) 2:801, [y]3:282, [w]8:23.

\subsection{Binomial $(1, \mathrm{p})$}

$\mathrm{D}(\mathrm{x})=\mathrm{p}^{\mathrm{x}}(1-\mathrm{p})^{1-\mathrm{x}}, \quad \mathrm{x}=0,1$.

$\mathrm{D}(\mathrm{n} \overline{\mathrm{x}})=$ Binomial $(\mathrm{n}, \mathrm{p}): \quad[6] 20 \%,[w] 1: 73$.

Chi-square test: $[e] 13: 3$.

Confidence intervals for $\mathrm{p}: \quad[6] 233$.

Completeness: $\quad[e] 10: 315$.

UMVUE $(p)=\bar{x}, \quad$ UMVUE $(p q)=n \bar{x}(1-\bar{x}) /(n-1)$.

See also: $\quad[v] 3: 324,[w] 1: 9$.

3.3 Truncated Binomial (k, p)

$\mathrm{D}(\mathrm{x})=\left(\begin{array}{l}\mathrm{k} \\ \mathrm{x}\end{array}\right)\left(\mathrm{p}^{\mathrm{x}} \mathrm{q}^{\mathrm{k}-\mathrm{x}} /\left(1-\mathrm{q}^{\mathrm{k}}\right)\right), \quad \mathrm{x}=1, \ldots, \mathrm{k}: \quad[6] 162$.

$\mathrm{E}(\mathrm{x})=\mathrm{kp} /\left(1-\mathrm{q}^{\mathrm{k}}\right), \quad \mathrm{v}=\mathrm{kpq} /\left(1-\mathrm{q}^{\mathrm{k}}\right)$, moments of $\mathrm{x}^{-\mathrm{p}}: \quad[d] 16: 50$.

Estimation: $[g] 50: 87 \%$.

Tables: $[g]$ 49:169,

With an application: [k]14:321.

See also: $\quad[b] 11: 2, M R 15: 969$.

3.4. Negative Binomial $(k, p)$

\begin{tabular}{|c|c|c|c|c|c|}
\hline $\mathrm{D}(\mathrm{x})$ & $\mathrm{p}$ & $q=1-p$ & Mean & Variance & Reference \\
\hline$\left(\frac{-k}{x}\right) \mathrm{p}^{\mathrm{x}}(1-\mathrm{p})^{-\mathrm{k}-\mathrm{x}}$ & $\mathrm{p}$ & $1-p$ & $-\mathrm{kp}$ & $-\mathrm{kpq}$ & \\
\hline$\left(\frac{\mathrm{a}}{1+\mathrm{a}}\right)^{\mathrm{k}}\left(\frac{-\mathrm{k}}{\mathrm{x}}\right) \frac{(-1)^{\mathrm{x}}}{(1+\mathrm{a})^{\mathrm{x}}}$ & $-1 / \mathrm{a}$ & $\frac{a+1}{a}$ & $\mathrm{k} / \mathrm{a}$ & $\mathrm{k} / \mathrm{a}+\mathrm{k} / \mathrm{a}^{2}$ & $\begin{array}{l}{[1] 259} \\
{[a] 83: 255}\end{array}$ \\
\hline coefficient of $t^{\mathrm{x}}$ in $\left(\frac{\mathrm{a}}{1+\mathrm{a}}\right)^{\mathrm{k}}\left(1-\frac{\mathrm{t}}{1+\mathrm{a}}\right)^{-\mathrm{k}}$ & $-1 / \mathrm{a}$ & $\frac{a+1}{a}$ & $\mathrm{k} / \mathrm{a}$ & $\mathrm{k} / \mathrm{a}+\mathrm{k} / \mathrm{a}^{2}$ & [2]125 \\
\hline $\mathrm{p}^{\mathrm{k}}\left(\begin{array}{c}\mathrm{x}+\mathrm{k}-1 \\
\mathrm{k}-1\end{array}\right)(1-\mathrm{p})^{\mathrm{x}}$ & $\frac{p-1}{p}$ & $1 / \mathrm{p}$ & $\mathrm{qk} / \mathrm{p}$ & $\mathrm{qk} / \mathrm{p}^{2}$ & $\begin{array}{c}{[d] 17: 53,[6] 61,} \\
{[18] 1-158,} \\
{[17] \text { No. } 6,} \\
{[7] 218}\end{array}$ \\
\hline$\left(\begin{array}{c}x+k-1 \\
k-1\end{array}\right) \frac{p^{x}}{(1+p)^{k+x}}$ & $-\mathrm{p}$ & $1+\mathrm{p}$ & $\mathrm{p}$ & $\mathrm{p}+\mathrm{p}^{2}$ & {$[f] 9: 176,[4] 54$} \\
\hline$\left(\frac{\mathrm{m}}{1+\mathrm{bm}}\right)^{\mathrm{x}}(1+\mathrm{bm})^{-1 / \mathrm{b}} \frac{1}{\mathrm{x} !} \prod_{\mathrm{j}=1}^{\mathrm{x}-1}(1+\mathrm{jb})$ & $-b m$ & $1+b m$ & $\mathrm{~m}$ & $\mathrm{~m}(1+\mathrm{bm})$ & {$[5] 32$} \\
\hline$\left(\frac{n}{n+k m}\right)^{n}\left(\begin{array}{c}x+n-1 \\
n-1\end{array}\right)\left(\frac{k m}{n+k m}\right)^{x}$ & $-\mathrm{km} / \mathrm{n}$ & $\frac{\mathrm{n}+\mathrm{km}}{\mathrm{n}}$ & $\mathrm{km}$ & $\mathrm{km}+\frac{\mathrm{k}^{2} \mathrm{~m}^{2}}{\mathrm{n}}$ & {$[c]_{4} 1: 78$} \\
\hline
\end{tabular}


If $\mathrm{Qp}=1, \quad \mathrm{Q}(1-\mathrm{p})=\mathrm{P}$, then $\beta_{1}=(\mathrm{P}+\mathrm{Q}) / \sqrt{\mathrm{kPQ}}$, $\beta_{2}=3+(1+6 \mathrm{PQ}) / \mathrm{kPQ}$.

Obtained by assuming a Poisson parameter to be Type III: [1]259, [2]125, [c]41:78, [a]110:132, [f]5:162.

Some derivations, with interesting properties: $[18] 1-159,[c] 44: 530$.

If $\mathrm{k}=\mathrm{h} / \mathrm{p}$, called Polya-Eggenburger: [4]55.

Ch(x): $\quad[17]$ No. \%, extension, Mem. Fac. Sci. Kyushu Imp. Univ. (Ser A) 1:178.

A "contagious" distribution: [7]83, 101, [13]/413, [7]128.

Skewness, kurtosis, cumulants: [18]1-136, 1144 .

$\mathrm{C}(\mathrm{x})=(\mathrm{p}+\mathrm{q})^{-\mathrm{n}}: \quad[c] 37: 209$.

$\mathrm{Ch}(\mathrm{x})=\left[1+\operatorname{bm}\left(1-\mathrm{e}^{\mathrm{it}}\right)\right]^{-1 / \mathrm{b}}: \quad$ [5]62.

Called compound Poisson: [15] $72 \%$.

$\mathrm{Ch}(\mathrm{x}): \quad[v]_{4}: 9$.

Limit of contagious: $\quad[c] 41: 269$.

Limited by Poisson and Pascal: [7]233.

PGF: $\quad[18] 1-146$.

Problem leading to Negative Binomial, with generalization: $\quad[d] 17: 53$.

Generalization: MR16:602.

Paper by Fisher: $\quad[k] 11: 182$.

Recurrence formula for cumulants: Aktuárské Vèdy 5:182.

Moments: G. Dell Ist. Ital. degli Attuari 6:3, Z13:70.

Formulas for tails: [7]23\%.

MLE: $\quad[b] 8: 206,[c] 37: 114$.

$\mathrm{M} \chi^{2} \mathrm{E}: \quad[k] 11: 109$.

UMVUE $(p): \quad[e] 18: 3 \%$.

Estimation: [d]24:409, Psychometrika 16:10\%.

Sequential: $\quad[f] 6: 59$.

Sampling: $[c] 37: 358$.

Fitting: $\quad[f] 9: 176$.

Truncated: $[g] 50: 8 \%$.

Moments, estimation, MLE: [c] 42:58.

Bhattacharyya bounds: $[d] 2 \%: 1182$.

Transformation $\sinh ^{-1} \mathrm{x}: \quad[f] 3: 52,[c] 35: 249$.

Transformations: $[c] 41: 315$.

Called "Pascal," satisfies:

$\mathrm{D}(\mathrm{x}+1)=[(1-\mathrm{p})(\mathrm{x}+\mathrm{k}) /(\mathrm{x}+1)] \mathrm{D}(\mathrm{x})$, etc.

Accident proneness: $[c] 37: 24$.

$[i] 14: 176$.

Telephone traffic: $[j] 35: 454$.

Bibliography: $[1] 43 \%$.
See also: [7]236, [b]10:260, [f]5:165, [f]7:340, $411,[c] 35: 11,[c] 39: 178,198,[c] 40: 203,[c] 40: 370$, $[c] 44: 364,[i] 20: 78,[i] 22: 25,[i] 31: 9, J$. D'Analyse Math 1:331, Psych. Bull., 47:434, [u]45:364, Z6:69, Z18:265. Z13:409, Z14:29, MR17:944, [a]99:733, [w]8:23.

\subsection{Negative Binomial $(1,-\mathrm{m})$}

$\mathrm{D}(\mathrm{x})=[1 /(1+\mathrm{m})][\mathrm{m} /(1+\mathrm{m})]^{\mathrm{x}}$, "Pascal," or "Furry," or "Geometric" $\alpha_{1}=\mathrm{m}, \quad \mathrm{V}=\mathrm{m}^{2}+\mathrm{m}$,

$\mathrm{Ch}(\mathrm{x})=\left[1+\mathrm{m}\left(1-\mathrm{e}^{\mathrm{it}}\right)\right]^{-1}: \quad[5] 31,60,66$.

Cumulants: $\quad[18] 1-144$.

PGF: $\quad[18] 1-148$.

If $\mathrm{D}\left(\frac{\mathrm{m}}{1+\mathrm{m}}\right)=$ Beta, then $\mathrm{D}(\mathrm{x})=$ No. 8.93

See also: $[c] 39: 346,[7] 59,[v] 4: 8, \quad[c] 36: 165$, $[15] 38,[c] 44: 265$.

\subsection{Discrete Lexian}

$\mathrm{D}(\mathrm{x})=\Sigma \mathrm{f}(\mathrm{p})\left(\begin{array}{l}k \\ x\end{array}\right) \mathrm{p}^{\mathrm{x}}(1-\mathrm{p})^{\mathrm{k}-\mathrm{x}}$, moments, etc. :

$[i] 26: 34$.

"Generalized Binomial".

Poisson-Lexian: [i]26:5\%.

$\mathrm{Ch}(\mathrm{x})=[\mathrm{p} \phi(\mathrm{t})+\mathrm{q}]^{\mathrm{k}}: \quad[17]$ No.69.

If a priori distribution of $\mathrm{p}$ is Beta:

Am. Math. Soc. $41: 860$.

[i]2\%:39, Bull.

\subsection{Deterministic}

$\mathrm{D}(\mathrm{x})=\left\{\begin{array}{l}0, \mathrm{x} \neq \mathrm{c} \\ 1, \mathrm{x}=\mathrm{c}\end{array}\right\}:$ "casual" $\quad[1] 192$.

$\mathrm{Ch}(\mathrm{x})=\mathrm{e}^{\mathrm{ict}}: \quad[8] 209,[5] 29,62$.

For $\mathrm{c}=1$ : [2]96.

Bibliography: $[1 \%]$ No. 1 .

Moments, cumulants, Ch(x), PGF: [18]1-136, $1-144,1-146,[v] 3: 324$.

See also: $\quad[c] 44: 366,29: 363,[w] 1: 9$.

\subsection{Reciprocal Truncated Binomial}

$\mathrm{D}(\mathrm{x}): \quad[n] 18$ No. 1-2:7\% .

\section{Discrete Distributions}

\subsection{Poisson $(\mathrm{m})$}

$\mathrm{D}(\mathrm{x})=\mathrm{e}^{-\mathrm{m}} \mathrm{m}^{\mathrm{x}} / \mathrm{x} !, \quad \mathrm{x}=0,1, \ldots . \quad$, law of small numbers: $[6] 59,[5] 30,[8] X,[7] 72,115,[10] 47$, 63, [15]119.

$\mathrm{Ch}(\mathrm{x})=\exp \left[\mathrm{m}\left(\mathrm{e}^{\mathrm{it}}-1\right)\right]: \quad[1] 204,[5] 62,[2] 66$.

$\left.\operatorname{MGF}(\mathrm{x})=\mathrm{e}^{-\mathrm{m}} \exp \left(\mathrm{me}^{\mathrm{t}}\right): \quad[6] 101,[4] 53, \mathrm{i}^{\mathrm{m}}\right] 2: 46$.

$\alpha_{1}=\mathrm{m}, \quad \alpha_{2}=\mathrm{m}^{2}+\mathrm{m}, \quad \mathrm{v}=\mathrm{m}: \quad$ [6]102, [5]5\%, 66, [4]53.

$\alpha_{3}=\mathrm{m}\left[(\mathrm{m}+1)^{2}+\mathrm{m}\right]$,

$\alpha_{4}=\mathrm{m}\left(\mathrm{m}^{3}+6 \mathrm{~m}^{2}+7 \mathrm{~m}+1\right): \quad[10] 59$. 
$\mu_{2}=\mathrm{m}, \quad \mu_{3}=\mathrm{m}, \quad \mu_{4}=\mathrm{m}(1+3 \mathrm{~m})$,

$\mu_{5}=\mathrm{m}(1+10 \mathrm{~m}), \quad \mu_{6}=\mathrm{m}\left(1+25+15 \mathrm{~m}^{2}\right):$

Skewness, kurtosis: $\quad$ [18]1-136.

[d]1:119, [2]86.

PGF : $\quad[18] 1-1.46$.

$\mu_{\mathrm{r}+1}=\mathrm{rm} \mu_{\mathrm{r}-1}+\mathrm{m}\left(\mathrm{d} \mu_{\mathrm{r}} / \mathrm{dm}\right):$

Math. Soc. 40:26\%, $41: 85 \%$.

[2]121, Bull. Am.

All cumulants $=\mathrm{m}: \quad$ [2] 66 .

Factorial moments $\alpha_{[1]}=\mathrm{m}^{\mathrm{i}}: \quad$ [1]25\%.

Moments in general: $[d] 8: 103,[i] 14: 173$.

Recursion formula for moments, correction with multinomial, $\mathrm{C}(\mathrm{x})$ as a $\Gamma$ integral: $\quad[1 / 4] 36-8$.

$\beta_{1}=1 / \mathrm{m}, \quad \beta_{2}=3+1 / \mathrm{m}, \quad \gamma=1 / \mathrm{m}$ : [12]52.

$\mathrm{C}(\mathrm{x}): \quad[j] 5: 604, M R_{4}: 194,[c] 37: 313$.

Transform $y=\sqrt{x}: \quad[14] 209$.

$\mathrm{D}(\overline{\mathrm{x}})=\mathrm{e}^{-\mathrm{nm}}(\mathrm{nm})^{\mathrm{n} \overline{\mathrm{x}}} /(\mathrm{n} \overline{\mathrm{x}}) !: \quad[1] 379,[2] 243,[6] 208$, [15]219.

$\mathrm{D}(\mathrm{x}+\mathrm{y}): \quad[10] 59$.

$\sim \mathrm{D}\left(\frac{\mathrm{x}-\mathrm{m}}{\mathrm{m}}\right)=\mathrm{N}\left(\mathrm{m}, \mathrm{m}^{\frac{1}{2}}\right): \quad[1] 250$.

$\mathrm{D}(\mathrm{x}-\mathrm{y}):$ [2]251, MR14:566, [a]109:296, [a]100: $415,[v] 7: 175$.

$\sim \mathrm{D}(\mathrm{x}-\mathrm{y}): \quad$ MR15:138.

$\mathrm{D}$ (gap between two Poisson events)=exponential: [c]41:252, [g].49:255, MR14:293, Am. Math. Monthly $64: 719$.

$\mathrm{D}\left[\overline{\mathrm{x}}^{-1} \Sigma\left(\mathrm{x}_{\mathrm{i}}-\overline{\mathrm{x}}\right)\right]$, i.e., Chi-square test:

$[b] 5: 75$, Z18:321.

$\mathrm{D}$ (Index of dispersion) :

$[d] 14: 155,[c] 40: 225$.

Various a priori distributions of $\mathrm{m}$, in particular Type III: $\quad[i] 27: 33$.

$\mathrm{E}\left(\mathrm{x}^{2}\right)=\mathrm{E}(\mathrm{x}+1): \quad[8] 119$.

$\mathrm{E}(1 / \mathrm{x}): \quad[g] 49: 169$.

$\operatorname{Var}\left(\mathrm{x}^{\frac{1}{2}}\right):\lfloor a] 106: 143$.

Reproductive property by convolutions: [7]216. by Ch. functions: [9]279.

C. - R. $(\mathrm{m})=\mathrm{m} / \mathrm{n}: \quad[1] 48 \%$.

C. - R. $\left(\mathrm{m}^{2}\right)=4 \mathrm{~m}^{3} \mathrm{n}^{-1}$.

BANE: $\quad[d] 21: 401$.

Estimation when m must be integral: $\quad[b] 12: 213$

Estimation: $[d] 24: 406$.

Estimation from censored samples: $[g] 49: 158$.

Estimation of bacteria population: $[c] 31: 170$.

Estimation of $\mathrm{m}$ or $1 / \mathrm{m}: \quad[\mathrm{g}] 49: 255$.

$\mathrm{M} \chi^{2} \mathrm{E}: \quad[3] 56$.

Approximation, estimation, application: $[15]^{7 / 4}$.

$\operatorname{MLE}(\mathrm{m})=\overline{\mathrm{x}}: \quad[4] 141,[9] 6.22, \quad[5] 144,[3] 21,[p]$ $7: 169$.

$\overline{\mathrm{x}}$ is sufficient: [4]136, [w]22:713. $\overline{\mathrm{x}}$ is efficient: $\quad[1] 48 \%$.

$\overline{\mathrm{x}}$ is unbiased: [3]142.

Completeness: $\quad[e] 10: 315$.

Confidence intervals, tail: $\quad[c] 41: 312$.

C.-R: $[16] 16$.

Confidence intervals: $[3] \% 1,81,[d] 9: 173,[c] 28: 43 \%$, $[c] 44: 436,[e] 14: 25,[p]^{7}: 223$.

Order statisties: MR16:799.

Approximate moments of ordered variables: $[s]$ $8: 78$.

Testing whether two Poissons are the same: [3]127, $[c] 37: 143$.

Two Poissons, etc.: $\quad[c] 40: 44 \%$.

Whether $\mathrm{k}$ Poissons are the same: [d]16:362, Proc. Nat. Inst. Sci. India 3:297.

Analysis of variance: $[d] 11: 335, J$. Econ. Entom. $37: 71 \%$.

Testing m: $[c] 31: 314,[c] 40: 354,[g] 49: 255,[14] 205$.

Testing ratios of means: $[0] 4: 45$.

Chi-square test: $[k] \%: 20 \%$.

Testing against contagious: $\quad[c] 37: 59$.

Sequential testing $\mathrm{m}: \quad[d] 19: 400$.

Small sample tests: $\quad[f] 12: 264$.

Monograph on Poisson testing and estimation: MR16:383.

Obtained from a difference equation analogous to Pearson's differential equation: $\quad[f] 2: 419$.

Obtained from postulates: $\quad$ Z13:408.

Early discussion with numerical examples: $[c]$ $10: 36$.

Transform $\quad \mathrm{y}=(\mathrm{x}+\mathrm{k})^{\frac{1}{2}}: \quad[d] 14: 113, \quad[f] 3: 52, \quad[c]$ 35:24\%.

Transformations: $\quad[c] 41: 312$.

Domain of attraction: MR3:2.

As limit of binomial: MR12:190, [4]52, [7]110, $[i] 6: 78,[s] 1: 161$.

Generalizations: [c]36:18, Operations Res. 3:198, $[c] 37: 48$.

A modification: [e]15:237, 251 .

Convergent sequence of Poissons: Am. Math. Monthly 50:97.

Connection with hypergeometric: $[c] 25: 300$.

If $\mathrm{m}$ Poisson, called double Poisson: Kendall and Buckland, A Dictionary of Stat. Terms.

Normal approximation: [7]146, MR16:1034, MR10:613, $[r] 4: 3 \%$.

Compounded with binomial: [7]128,221.

As approximation to Beta: [j]20:19.

An approximation: MR18:423. 
Connection with Gram-Charlier: [2]154.

Connection with Type X: $\quad[0] 2: 13, D$. L. Gerlough and A. Schuhl, Poisson and Traffic, The ENO Foundation, 1955.

Connection with Type III: [o]3:123.

Limiting theorems: $[8] 148$.

Poisson if and only if $\sum_{1}^{\mathrm{a}} \mathrm{x} \mathrm{D}(\mathrm{x})=\mathrm{m} \mathrm{C}(\mathrm{a}-1)$

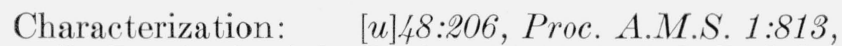
C. R. Acad. Sci. Paris 239:1114, 3rd Berkeley Symp 2:145.

Generalizations: $[d] 13: 410,[d] 14: 394$, MR15:138, MR16:1034, [d]19:414, MR13:258.

Possibility of a continuous analog: $\quad[i] 14: 43$.

Traffic control: $[b] \%: 65$.

Accident causation: $\quad[b]^{\gamma}: 89,[a] 90: 48 \%$.

Poisson as a limiting distribution in five different ways, relation with multinomial, exponential: [18]1-156.

Accident proneness: $\quad[c] 37: 24$.

Pedestrian delay: $[c] 38: 383$.

Insurance risk: $\quad[i] 40: 72$.

Frequency of war: $\quad[a] 107: 242,[a] 112: 446$.

Nomograph for acceptance inspection: $[s]_{4}: 204$.

Telephone switchboards: [5]30, [j]6:468.

An early treatment, with the famous example of the Prussian horse-kicks: Von Bortkewjtsch, L. Das Gesetz der Kleinen Zahlen, B. G. Teubner, Leipzig 1898.

Other applications: $\quad[7] 119,[j] 5: 604$.

See also: $\quad[d] 14: 155,[d] 20: 523,[d] 22: 94,[d] 22: 128$ $[13] 405,[j] \%: 45,[c] 11: 267,[c] 11: 211,[c] 26: 108,[g]$ 33:390, [g] 42:574, [a]83:255, [f]6:17, MR1:246, Z22:243, MR17:53, MR14:485, Z18:31, Z14:138, MR4:20, MR13:633, MR15:541, 634, MR':310, $[f] \%: 340,[c] 27: 272,[c] 30: 188,[c] 36: 250,[n] 16-2: 285$, $[k] 9: 406,[c] 38: 427,[c] 39: 346,[i] 20: 80,[i] 22: 25,[i]$ 25:158, [i]26:46, [i]31:9, Rio. Ital. di Demogr. e Stat 3:219, Proc. First Pakistan Stat. Conf. (1950) p. 59, Publ. Math. Debrecen 2:66, Annals of Applied Biology 9:325, Annales de l'Ecole Normalle Superiere 54:321, $[v] 2: 330,[v] 3: 327,[d] 26: 147,[c] 44: 265,365$, Brit. Assoc. Math. Tables (3rd Ed.) V.1 p.xxxvi, Am. Math. Monthly 50:97, Annals of Math 37:357, Bull. Am. Math. Soc. 1935 p. 861, Am. J. Math 57:827, [u] 45:219, Kungl. Lantbruk. Ann. 18:86, Z2:200, MR1:15, MR18:341, MR5:128, MR2:112, Z15:407, Z18:412, MR14:1098, MR13:958, [w]1:9, [s]1:93.

\subsection{Truncated Poisson}

$\mathrm{D}(\mathrm{x})=\mathrm{m}^{\mathrm{x}} \mathrm{e}^{-\mathrm{m}} / \mathrm{x} !\left(1-\mathrm{e}^{-\mathrm{m}}\right), \quad \mathrm{x}=1,2, \ldots$.

$[f] 8: 275,[f] 10: 402,[f] 11: 38 \%$.
Estimation: $[c] 39: 24 \%,[c] 40: 171,[g] 50: 906,[i]$ $39: 19,[f] 9: 485$.

UMVUE: $\quad[e] 18: 3 \% 4$.

Tables: $[g]$ 49:169, [11]158, [13]39:24\%.

Servicing machines: $[b] 13: 71$.

$\mathrm{D}(1 / \mathrm{x}): \quad[n] 18$ No. 1-2:7\%.

Doubly truncated, $\mathrm{D}(\mathrm{x})$ etc: [g] 49:160, Conn. Agric. Exp. Sta. Bull. No. 513.

\subsection{Compound Poisson}

$\mathrm{D}(\mathrm{x})=\mathrm{k}_{1}\left(\mathrm{e}^{-\mathrm{m}} \mathrm{m}^{\mathrm{x}} / \mathrm{x} !\right)+\mathrm{k}_{2}\left(\mathrm{e}^{-\mathrm{n}} \mathrm{n}^{\mathrm{x}} / \mathrm{x}\right.$ !): double Poisson: [5]151.

If $\mathrm{k}_{1}=\mathrm{k}_{2}=\frac{1}{2}: \quad[5] 150,[g] 42: 40 \%,[f] 8: 281$.

Compound Poisson $\mathrm{D}(\mathrm{x})=(1 / \mathrm{x} !) \Sigma \mathrm{m}_{1}^{\mathrm{x}} \mathrm{e}^{-\mathrm{m}_{\mathrm{i}}} \mathrm{k}_{1}$ :

237, MR17:862, R. D. Evans, The Atomic Nucleus, p. 766, MR13:633, MR14:7\%0.

\subsection{Uniform}

$\mathrm{D}(\mathrm{x})=1 / \mathrm{k}, \quad \mathrm{x}=1, \ldots, \mathrm{k}: \quad[6] 61$.

Discrete rectangular.

MGF and moments: $\quad[d] 11: 324$.

Sampling from: $\quad[c] 21: 126$.

Estimation of range: $[g] 46: 375$.

Range and quotient of ranges: Int. Congr. Math. (1950) $1: 583$.

$\mathrm{D}(\mathrm{n} \overline{\mathrm{x}}): \quad[p] 10: 52,255,324$.

See also: MR16:376.

\subsection{Hypergeometric}

$\mathrm{D}(\mathrm{x})=\left[\left(\begin{array}{c}\mathrm{m} \\ \mathrm{x}\end{array}\right)\left(\begin{array}{c}\mathrm{n} \\ \mathrm{r}-\mathrm{x}\end{array}\right)\right] /\left(\begin{array}{c}\mathrm{m}+\mathrm{n} \\ \mathrm{r}\end{array}\right): \quad[6] 61,[7] 33,[15] 40$.

$\mathrm{D}(\mathrm{x})$ in another form, moments, etc: [2]126.

In the form of a hypergeometric series, asymptotic forms: $\quad[c] 25: 295,[c] 26: 59$.

Various forms: J. Soc. Stat. Paris 96:262.

$\mathrm{C}(\mathrm{x})$ as a power series: $[c] 41: 31 \%$.

Ch $(x)$, refs: $\quad[17]$ No. 8 .

$\mathrm{E}(\mathrm{x})=\mathrm{mr} /(\mathrm{m}+\mathrm{n}), \quad \mathrm{v}=[\mathrm{mnr}(\mathrm{m}+\mathrm{n}-\mathrm{r})] /\left[(\mathrm{m}+\mathrm{n})^{2}\right.$ $(\mathrm{m}+\mathrm{n}-1)]: \quad[\mathrm{y}] 183,[6] 98$.

Difference equation, moments, etc: $\quad[i] 14: 178$.

Factorial moments: [2]135, Nature 164:282, [i] $6: 79$

Skewness: $\quad[18] 1-136$.

PGF: $\lfloor 18] 1-146$.

Moments in general: $[d] 8: 103,[d] 10: 198, \quad[c]$ $16: 15 \%,[c] 17: 5 \%,[c] 26: 264,[a] 89: 326,[v] 3: 326$, GANITA $\%: 1$.

Binomial and Poisson as limits: [15]690.

Binomial as limit: $[7] 4 \%$. 
Poisson as limit: $[7] 114,[c] 25: 300$.

Normal as limit: $\quad[7] 146$.

Normal, Poisson, binomial approximations: $1-155,[d] 2 \%: 471$.

Minimax estimation: $[d] 21: 191$.

Completeness: $\quad[e] 10: 315$.

"Confidence Limits for the Hypergeometric Distribution: Chung and DeLury Univ. of Toronto Press, 1950, reviewed, [a]115:286.

Generalization: $\quad[\boldsymbol{\gamma}] 39,[c] 41: 266$, see also No. 8.59, [b]18:202.

Satisfying difference equation $[w] 3: 5$

Linguistic application: [b]12:2\%.

Truncated hypergeometric, moments: [d]16:59.

Double hypergeometric: $\quad[\%] 18 \%$, Koninkl. Nederl. $\operatorname{Akad}(A)$ 60:121.

See also: $\quad[d] 1: 113,[d] 21: 248,[e] 11: 153,[j] 7: 39$, $[j] 10: 281,[f] 8: 28 \%,[n] 1-4: 49,[c] 37: 140,[h] 2: 435$, [i]22:26, [d]25:762, J. Proc. Roy. Soc. N.S.W. 81:38, N.B.S. Math. Table MT19, MR17:984, Z12:29, Z13:273, MR1:340, U. Calif. Pub. Stat 1, No. 7, MR13:962, MR14:775, [w]1:9.

\subsection{Contagious}

Obtained by considering $\mathcal{S}$ (Poisson) $\mathrm{d} \mathbf{F}(\mathrm{m})$ :

MR14:293, [d]14:389, [v]8:13.

$\mathrm{F}$ step function yields $\mathrm{D}(\mathrm{x})=(1 / \mathrm{x} !) \Sigma \mathrm{p}_{\mathrm{i}} \mathrm{e}^{-\mathrm{a}_{\mathrm{i}}} \mathrm{a}_{\mathrm{i}}^{\mathrm{x}},\left(N_{o}\right.$. 4.3)

F Type III yields Polya-Eggenberger

$$
\mathrm{D}(\mathrm{x})=\frac{1}{\mathrm{x} !} \frac{\Gamma(\mathrm{x}+\mathrm{h} / \mathrm{d})}{\Gamma(\mathrm{h} / \mathrm{d})}(1+\mathrm{d})^{-\mathrm{d} / \mathrm{h}}(1+\mathrm{d})^{-\mathrm{x}} .
$$

$\mathrm{m}$ itself also Poisson yields

$\mathrm{D}(\mathrm{x})=\mathrm{e}^{-\mathrm{k}}\left(\mathrm{c}^{\mathrm{x}} / \mathrm{x}\right.$ !) $\Sigma\left(\mathrm{i}^{\mathrm{x}} / \mathrm{i}\right.$ ! $)\left(\mathrm{e}^{-\mathrm{c}} \mathrm{k}\right)^{\mathrm{i}}$.

Neyman contagious Type A: $\quad[c] 40: 208$.

Fitting: [f]11:149, F. N. David, Probability Theory for Statistical Methods, p. 68, Cambridge 1949.

Ch. fens: $[g] 49: 368$.

Generalization: $[f] 9: 354$.

Testing against Poisson: $\quad[c] 37: 59$.

Rutherford contagious: [d]25:703.

$\Sigma_{\mathrm{j}}$ (Poisson) $\mathrm{f}_{\mathrm{j}}(\mathrm{x}), \mathrm{Ch}(\mathrm{x})$, sp. cases: $\quad[17]$ Nos. 66-8. Bivariate: MR15:138.

See also: $[13] 411,[f] 9: 354,[c] 36: 450,[c] 39: 346$, $[c] 40: 186,[c] 41: 268,[d] 10: 35, J$. Econ. Entom. 35: 536, MR1:251.

\subsection{Pollaczek-Geiringer}

$\mathrm{D}(\mathrm{x}), \mathrm{Ch}(\mathrm{x})$,

multiple occurrence of rare events:
4.8. Borel-Tanner

$\mathrm{D}(\mathrm{x})=\left(\mathrm{e}^{-\alpha \mathrm{x}} \alpha^{\mathrm{x}-\mathrm{r}} \mathrm{x}^{\mathrm{x}-\mathrm{r}-1} \mathrm{r}\right) /(\mathrm{x}-\mathrm{r}) !, \mathrm{x}=\mathrm{r}, \mathrm{r}+1, \ldots$, $0 \leq \alpha \leq 1, \quad \mathrm{r}=1,2, \ldots . . \quad$ C.R. Acad. Sci., Paris $214: 452,[c] 40: 58,[c] 47: 143$.

$\mathrm{m}=\mathrm{r} /(1-\alpha), \mathrm{v}=\left[\alpha \mathrm{r} /(1-\alpha)^{3}\right]$.

If $\mathrm{r}=1, \operatorname{MLE}(\alpha)=(\overline{\mathrm{x}}-1) / \overline{\mathrm{x}}$.

$\operatorname{PGF}(\mathrm{x})=\mathrm{t}^{\mathrm{r}} \exp \mathrm{r} \sum_{\mathrm{n}=1}^{\infty}\left(\mathrm{n}^{\mathrm{n}-1} \alpha^{\mathrm{n}} / \mathrm{n} ! \mathrm{e}^{\alpha \mathrm{n}}\right)\left(\mathrm{t}^{\mathrm{n}}-1\right)$.

\subsection{Polya}

$D(x)=\left(\begin{array}{c}N \\ x\end{array}\right) \frac{\prod_{i=0}^{x-1}(m+i R) \prod_{j=0}^{N-x-1}(n-m+j R)}{\prod_{k=0}^{N-1}(n+k R)}:$

$[\%] 128$

[d]28:1021.

Contains Polya-Eggenburger No. 3.4, and Exceedance No. 4.10.

\subsection{Exceedance}

$\mathrm{D}(\mathrm{x})=\frac{\left(\begin{array}{c}\mathrm{n} \\ \mathrm{m}\end{array}\right) \mathrm{m}\left(\begin{array}{c}\mathrm{N} \\ \mathrm{x}\end{array}\right)}{(\mathrm{n}+\mathrm{N})\left(\begin{array}{l}\mathrm{N}+\mathrm{n}-1 \\ \mathrm{~m}+\mathrm{x}-1\end{array}\right)}, \quad \mathrm{x}=0,1, \ldots, \mathrm{N}:$

$[d] 25: 762,[w] 6: 164,[d] 28: 1021,[d] 21: 247$.

Moments: $\quad[w] 6: 165$.

4.11. Inverse Hypergeometric

$\mathrm{D}(\mathrm{x})=\frac{\left(\begin{array}{c}\mathrm{n} \\ \mathrm{m}-1\end{array}\right)\left(\begin{array}{c}\mathrm{N}-\mathrm{n} \\ \mathrm{x}-\mathrm{m}\end{array}\right)(\mathrm{n}-\mathrm{m}+1)}{\left(\begin{array}{c}\mathrm{N} \\ \mathrm{x}-1\end{array}\right)(\mathrm{N}-\mathrm{x}+1)}$, estimation, truncation: Kungl. Lantbuck. Ann. 18:123.

\subsection{Generalized Poisson}

$\mathrm{D}(\mathrm{x})=\mathrm{e}^{-\lambda} \sum_{\mathrm{j}=1}^{\alpha} \frac{\lambda^{\alpha \mathrm{x}+\mathrm{j}-1}}{(\alpha \mathrm{x}+\mathrm{j}-1) !}$

Connected with Type III: $\quad[o] 3: 123,[0] 11: 101$.

\section{Distributions on $(a, b)$}

\subsection{Serial Correlation}

$\mathrm{D}(\mathrm{x})=\frac{\Gamma\left(\frac{1}{2} \mathrm{k}+1\right)}{\Gamma\left(\frac{1}{2} \mathrm{k}+\frac{1}{2}\right) \Gamma\left(\frac{1}{2}\right)}\left(1-\mathrm{x}^{2}\right)^{\frac{1}{2}(\mathrm{k}-1)}\left(1+\mathrm{r}^{2}-2 \mathrm{rx}\right)^{-\frac{1}{2} \mathrm{k}}$

$$
\begin{aligned}
& \alpha_{1}=\frac{\mathrm{rk}}{\mathrm{k}+2}, \\
& \mathrm{v}=(\mathrm{k}+2)^{-1}\left[1-\mathrm{r}^{2} \mathrm{k}(\mathrm{k}+1)(\mathrm{k}+2)^{-1}(\mathrm{k}+4)^{-1}\right]:
\end{aligned}
$$

$[c] 41: 261$.

[17]No.9. $\quad$ Moments, called "Leipnik": [c]44:270. 
See also: $[c] 35: 255,261,[d] 13: 1,14,[c] 43: 161$, 169, [d]18:86, Cowles Commission Papers, New Series No. 42 .

\subsection{Type I}

$\mathrm{D}(\mathrm{x})=\mathrm{C}(\mathrm{x}-\mathrm{a})^{\mathrm{p}-1}(\mathrm{~b}-\mathrm{x})^{\mathrm{q}-1}$, $\mathrm{a}<\mathrm{x}<\mathrm{b}, \mathrm{p}>0, \mathrm{q}>0$ : '[1]249, [17] No. 22.

Beta for $a=0, b=1$ normal for $\mathrm{p}=\mathrm{q}=\frac{1}{2} \mathrm{~b}^{2}, \mathrm{a}=-\mathrm{b}, \mathrm{b} \rightarrow \infty: \quad$ [2]139.

Type III for $\mathrm{b} \rightarrow \infty, \mathrm{q}=\alpha \mathrm{b}: \quad$ [1]249.

Obtained by assuming roots in quadratic of Pearson differential equation real with different sign: $[4] \% 4,[11] 43$.

Relations of various constants: [11]53, $[c] 16: 10 \%$.

Bayes' theorem: $[n] 16-1: 115$.

Fitting to observations: $[a] 96: 306,[c] 1: 31,[c] 1$ : 292, $[c] 1: 408,[c] 4: 474,[c] \%: 8 \%$.

Early volumes of $[c]$ give many examples with $\mathrm{D}(\mathrm{x})=\mathrm{C}(1+\mathrm{x} / \mathrm{a})^{\mathrm{m}}(1-\mathrm{x} / \mathrm{b})^{\mathrm{n}}$.

See also: $[d] 1: 148,[d] \%: 20,[d] 8: 1 \%,[d] 10: 15$, [c]3:311, [c]23:393, [c]26:386, [i] 16:53.

\section{3. $\operatorname{Beta}(p, q)$}

$\mathrm{D}(\mathrm{x})=\frac{\Gamma\left[\frac{1}{2}(\mathrm{p}+\mathrm{q})\right]}{\Gamma\left(\frac{1}{2} \mathrm{p}\right) \Gamma\left(\frac{1}{2} \mathrm{q}\right)} \mathrm{x}^{\frac{1}{2} \mathrm{p}-1}(1-\mathrm{x})^{\frac{1}{2} \mathrm{q}-1}, 0<\mathrm{x}<1, \mathrm{p}>-2$, q $>-2: \quad$ [2]139, [17]No. 21, [1]243, [10]153.

Called Beta distribution of the first kind: $[d] 25$ : 401.

$\alpha_{1}=\mathrm{p} /(\mathrm{p}+\mathrm{q}), \mathrm{v}=\mathrm{pq} /\left[(\mathrm{p}+\mathrm{q})^{2}\left(\frac{1}{2} \mathrm{p}+\frac{1}{2} \mathrm{q}+1\right)\right]:$ 41, [10]153, [14]42.

3d Moments : [2]419.

Mode $=(\mathrm{p}-2) /(\mathrm{p}+\mathrm{q}-4)$, etc. $: \quad[m] 2: 128$.

$\alpha_{\mathrm{r}}=\mathrm{B}(\mathrm{p}+\mathrm{r}, \mathrm{q}) / \mathrm{B}(\mathrm{p}, \mathrm{q}): \quad[6] 117,[b] 4: 126,[14] 42$.

$\mathrm{HM}=(\mathrm{p}-2) /(\mathrm{p}+\mathrm{q}-2), \quad[10] 163$.

$G M=\exp \left\{\left(\partial / \partial \frac{1}{2} p\right)\left[\log \Gamma\left(\frac{1}{2} p\right)-\log \frac{1}{2}(p+q)\right]\right\}$.

$\mathrm{C}(\mathrm{x}): \quad[d] 20: 451$.

Moments: [3]211.

Obtained as a Pearson Type: [4]\%4.

From an example: $[10] 45$.

$\mathrm{C}(\mathrm{x}): \quad[c] 19: 1,[c] 25: 379,[c] 38: 423,[c] 37: 208,[i] 38:$ 192.

$\mathrm{D}(\overline{\mathrm{x}}): \quad$ [2]251, $[n] 8-4: 55$.

When $\mathrm{p}=\mathrm{q}: \quad[c] 19: 230$.

Special cases and variants: [2]26.

$\operatorname{Beta}(\mathrm{n}-\mathrm{k}, \mathrm{k})$ : [1] 409 .

Beta $(2 \mathrm{n}-2,4)$ connected with tolerance limits: Hoel, Intro. to Math. Stat.

Correlation ratio in samples from uncorrelated bivariate normal is Beta $(\mathrm{k}-1, \mathrm{n}-\mathrm{k})$ : [2] 352, $[1] 414,[c] 21: 1,[c] 30: 290,[10] 181,184$.

$\mathrm{D}(1-\mathrm{x})=\operatorname{Beta}(\mathrm{q}, \mathrm{p})$

If $x$ is Beta $(p, q), y$ is Type III

$(1, p+q)$ then $x y$ is Type III $(1, p)$ :

$[10] 156$.

Standardized Beta variable is $\mathrm{N}(0,1)$ as

$\mathrm{p} \rightarrow \infty, \mathrm{q} \rightarrow \infty: \quad$ [1]252.

$\mathrm{D}(\mathrm{xy}): \quad[e] 9: 365$.

No sufficient statistic for $\left(\frac{1}{2} \mathrm{p}-1\right)$ : Proc. Roy. Soc. Lond. (Series A) 154:133.

Mellin transform: $[d] 19: 373$.

more generally: $\quad[b] 8: 136$.

$\log \log \frac{1}{\mathrm{x}}, \arcsin \sqrt{\mathrm{x}},: \quad[v] 8: 71$.

Generalized: $\quad[c] 43: 23 \%,[c] 44: 441$.

Transform: $[c] 36: 165$.

Connection with Fisher and Snedecor: [2]419, [c] $21: 350$.

Noncentral Beta: $\quad[d] 26: 648$.

Connection with binomial: $\quad[c] 41: 304,[n] 18: 121$.

Fitting straight lines: $[y] 24: 23$.

Approximated by Poisson: [j]20:19.

Range of rectangular $(0, a)$ is

$\mathrm{D}(\mathrm{x})=\mathrm{n}(\mathrm{n}-1) \mathrm{a}^{-\mathrm{n}} \mathrm{x}^{\mathrm{n}-2}(\mathrm{a}-\mathrm{x}): \quad$ [4]93, [c]25:417.

In trivariate normal analysis: [3]341.

$\operatorname{GM}\left(x_{1}, \ldots, x_{n}\right)$ and GM $\left(1-x_{1}, \ldots, 1-x_{n}\right)$ are joint sufficient: $[3] 49$.

In rank correlation: [2]418.

See also: $\quad[d] 16: 98,[d] 17: 325,[b] 1: 214,[c] 22: 284$, $[c] 22: 391,[c] 23: 143,[c] 27: 415,[10] 154,[c] 30: 140,[c]$ $33: 178,[c] 34: 368,[c] 35: 19,[c] 32: 151,271,[c] 36: 166$, $[k] 5: 75,[c] 39: 204,[c] 37: 219,[c] 40: 281,[g] 48: 831$, $[w] 1: 9$.

\subsection{Type II}

$\mathrm{D}(\mathrm{x})=\left[1-(\mathrm{x}-\mathrm{m})^{2} \mathrm{a}^{-2}\right]^{\mathrm{p}} /\left[\mathrm{aB}\left(\frac{1}{2}, \mathrm{p}+1\right)\right]$, $\mathrm{m}-\mathrm{a} \leq \mathrm{x} \leq \mathrm{m}+\mathrm{a}: \quad$ [2]141.

Properties: MR10:131.

Transform to Student: [c]28:308.

$\mathrm{D}(\overline{\mathrm{x}}): \quad[n] 10-3: 91$.

$\mathrm{C}(\mathrm{x}): \quad[c] 19: 12,[c] 25: 379$.

$\sim$ Dist. of rank correlation coefficient (Pitman): [c]30:259.

Called Thompson's distribution, relation with Student's, normal approximation: $[d] 27: 784$.

m location, a scaling, $\mathrm{p}$ shape, likelihood function: C. - R. $(\mathrm{m})$ Tables. $\mathrm{v}=\mathrm{a}^{2} /(2 \mathrm{p}+3): \quad[d] 3: 86$.

If $\mathrm{m}=0$

Distribution of Spearman's $\rho$ for large n: [2]401.

A numerical example: [11]62.

Estimation of center: $[t] 4: 33$.

From Pearson system: [11]43. 67 .

See also: $\quad[c] 4: 174,[c] 16: 114,[c] 21: 263,[n] 12-3:$ 
$\mathrm{D}(\mathrm{x})=\pi^{-\frac{1}{2}} \frac{\Gamma\left[\frac{1}{2}(\mathrm{n}-\mathrm{k}+1)\right]}{\Gamma\left[\frac{1}{2}(\mathrm{n}-\mathrm{k})\right]}\left(1-\mathrm{x}^{2}\right)^{\frac{1}{2}(\mathrm{n}-\mathrm{k}-2)}$,

$-1<\mathrm{x}<1: \quad[1] 412,[i] 24: 198$.

Type II with $\mathrm{m}=0, \mathrm{a}=1, \mathrm{p}=\frac{1}{2}(\mathrm{n}-\mathrm{k}-2)$.

$\mathrm{Ch}(\mathrm{x}): \quad[17]$ No. 19 .

If $\mathrm{k}=2$, transform to Student: [5]99.

If corresponding population parameter is zero: [10]256.

As No. 5.1 with $\mathrm{r}=0$ : Cowles Commission Papers, New Series No. 10.

If population is non-normal: [i] $36: 16$.

See also: $[d] 18: 81,[n] 2: 684,[o] 3: 45,[a] 92: 580$.

5.6. Parabolic

$\mathrm{D}(\mathrm{x})=\left[3\left(\mathrm{a}^{2}-\mathrm{x}^{2}\right)\right] / 4 \mathrm{a}^{3}, \quad-\mathrm{a}<\mathrm{x}<\mathrm{a}, \quad \mathrm{v}=\mathrm{a}^{2} / 5$, grouping corrections: $[c] 39: 432$.

Estimation: $\quad[d] 26: 505,[m] 6: 120$.

\subsection{Type IX}

$\mathrm{D}(\mathrm{x})=\frac{\mathrm{m}+1}{\mathrm{a}}(1+\mathrm{x} / \mathrm{a})^{\mathrm{m}},-\mathrm{a} \leq \mathrm{x} \leq 0$ :

[2]142, [17]No. 16

See also: $\quad[d] 7: 26,[c] 24: 234,240,263$.

Type VIII for negative $m$ :

[17]No. 23, MR4:21.

\subsection{Type XII}

$$
\begin{aligned}
\mathrm{D}(\mathrm{x})=(\mathrm{p} / \mathrm{q})^{m} \frac{(1+\mathrm{x} / \mathrm{p})^{\mathrm{m}}(1-\mathrm{x} / \mathrm{q})^{-\mathrm{m}}}{(\mathrm{p}+\mathrm{q}) \mathrm{B}(1+\mathrm{m}, 1-\mathrm{m})} \\
\quad|m|<1,-p \leq x \leq q:
\end{aligned}
$$

See also: $\quad[d]^{7}: 27,[17]$ No. 24.

\subsection{Correlation Determinant}

$$
\begin{aligned}
\mathrm{D}(\mathrm{x}) & =\frac{\Gamma\left[\frac{1}{2}(\mathrm{n}-1)\right]^{\mathrm{k}-1} \mathrm{x}^{\frac{1}{2}(\mathrm{n}-\mathrm{k}-2)}}{\pi^{\frac{1}{4} \mathrm{k}(\mathrm{k}-1)} \Gamma\left[\frac{1}{2}(\mathrm{n}-2)\right] \cdot . \cdot \Gamma\left[\frac{1}{2}(\mathrm{n}-\mathrm{k})\right]} \\
\alpha_{1}=(\mathrm{n}-1)^{1-\mathrm{k}}(\mathrm{n}-2)(\mathrm{n}-3) . & . \cdot(\mathrm{n}-\mathrm{k}), \\
\mathrm{v} & =\mathrm{k}(\mathrm{k}-1) \mathrm{n}^{-2}+0\left(\mathrm{n}^{-3}\right): \quad[1] 411,[4] 120 .
\end{aligned}
$$

Downton calls this "Geometric," and mentions, in connection with LSE, the following special cases: [d]25:304.

I. $\mathrm{D}(\mathrm{x})=\mathrm{px}^{\mathrm{p}-1}, 0 \leq \mathrm{x}<1$, $\mathrm{C}(\mathrm{x})=\mathrm{x}^{\mathrm{p}}, \alpha_{1}=\mathrm{p} /(\mathrm{p}-1), \mathrm{v}=\mathrm{p}(\mathrm{p}+2)^{-1}(\mathrm{p}+1)^{-2}$

II. $\mathrm{D}(\mathrm{x})=\mathrm{pb}^{-\mathrm{p}}(\mathrm{x}+\mathrm{a})^{\mathrm{p}-1},-\mathrm{a} \leq \mathrm{x}<\mathrm{b}-\mathrm{a}$

III. $\mathrm{D}(\mathrm{x})=\mathrm{pv}^{-\frac{1}{2}} \mathrm{~b}^{-\mathrm{p}}\left[(\mathrm{x}-\mathrm{m}) / \mathrm{v}^{\frac{1}{2}}+\mathrm{a}\right]^{\mathrm{p}-1}$,

$$
\begin{aligned}
& m-a v^{\frac{1}{2}} \leq x<m+(b-a) v^{\frac{1}{2}}, \alpha_{1}=m, \\
& a=p^{\frac{1}{2}}(p+2)^{\frac{1}{2}}, b=p^{-\frac{1}{2}}(p+1)^{3 / 2}, p \geq 1 .
\end{aligned}
$$

$\log \log ^{*}(\mathrm{x}): \quad[v] 8: 70$.
$\mathrm{D}(\mathrm{x})=1-|1-\mathrm{x}|, \mathrm{D}(\overline{\mathrm{x}})$ from rectangular when $\mathrm{n}=2$, $\mathrm{D}$ (range): $\quad[c] 25: 41 \%,[y] 24: 22, M R$ 3:171.

$\mathrm{D}(\mathrm{x})=\frac{2 \mathrm{x}}{2 \mathrm{k}+1}, \mathrm{k} \leqq \mathrm{x} \leqq \mathrm{k}+1: \quad[3] 47,[8] 32$.

Stratified sampling: $\quad[c] 13: 48$.

$\mathrm{D}(\mathrm{x})=(9 \sigma)^{-1}\left[\frac{\mathrm{x}-\mathrm{m}}{\sigma}+2 \sqrt{2}\right]$, right triangular

$\mathrm{m}-2 \sqrt{2} \sigma \leqq \mathrm{x}<\mathrm{m}+\sqrt{2} \sigma: \quad[d] 4: 256,[d] 25: 308$.

$\mathrm{D}(\mathrm{x})=4 \mathrm{R}^{-2}\left(\frac{1}{2} \mathrm{R}-|\mathrm{x}-\mathrm{m}|\right), \quad|\mathrm{x}-\mathrm{m}| \leqq \frac{1}{2} \mathrm{R}$, best linear estimate of $\mathrm{m}$ and $\sigma: \quad[d] 25: 318$.

Testing: $\quad[d] 25: 695$.

See also: $\quad[d] 2: 48,[d] 28: 179$.

$\mathrm{D}(\mathrm{x})=\frac{1}{\mathrm{a}}\left[1+\mathrm{k}-\frac{2 \mathrm{k}}{\mathrm{a}(\mathrm{a}-\mathrm{x})}\right], 0 \leqq \mathrm{x} \leqq \mathrm{a}$,

$-1 \leqq \mathrm{k} \leqq 1$. called "linear": $[d]_{4}: 244$.

$\mathrm{D}(\mathrm{x})=\left\{\begin{array}{l}1+\mathrm{x},-1 \leqq \mathrm{x}<0 \\ 1-\mathrm{x}, \quad 0 \leqq \mathrm{x}<1\end{array}\right.$, called "Tine": $\quad[d] 5: 33$.

$\mathrm{Ch}(\mathrm{x})=\left(2 / \mathrm{t}^{2}\right)(1-\cos \mathrm{t}): \quad[17]$ No. 14 .

$\mathrm{D}(\mathrm{x})=\frac{2}{\mathrm{a}^{2}}(\mathrm{a}-\mathrm{x})$, called "semi-triangular"

$\mathrm{m}=\mathrm{a} / 3, \mathrm{v}=\mathrm{a}^{2} / 18$, grouping corrections: $\quad[c] 39: 432$.

$\mathrm{Ch}(\mathrm{x}): \quad[17]$ No. 13.

Triangular on $(\mathrm{a}, \mathrm{b})$. If $\mathrm{x}$ and $\mathrm{y}$ are extreme values of the sample, then

$\mathrm{E}\left[\frac{1}{2}(\mathrm{x}+\mathrm{y})\right]=\frac{1}{2}(\mathrm{a}+\mathrm{b})$,

$$
\begin{aligned}
& \operatorname{Var}\left[\frac{1}{2}(x+y)\right]=\frac{4-\pi}{16 n}(b-a)^{2}+0\left(n^{-2}\right), \\
& E[(x-y)]=\left[1-\frac{\sqrt{\pi}}{2 n}\right](b-a)+0\left(n^{-3 / 2}\right), \\
& \operatorname{Var}(x-y)=\frac{4-\pi}{4 n}(b-a)^{2}+0\left(n^{-2}\right) .
\end{aligned}
$$

See also: $\quad[y] 16: 16$.

\subsection{Rectangular $(a-h, a+h)$}

$\mathrm{D}(\mathrm{x})=\frac{1}{2 \mathrm{~h}}, \mathrm{a}-\mathrm{h} \leq \mathrm{x} \leq \mathrm{a}+\mathrm{h} ; \mathrm{D}(\mathrm{x})=0$ elsewhere

$$
\mathrm{m}=\mathrm{a}, \mathrm{v}=\mathrm{h}^{2} / 3: \quad[1] 24 \text {. },[5] 34 .
$$

Skewness $=0$, kurtosis $=-6 / 5, \mathrm{~K}_{\mathrm{K}}, \mathrm{Ch}(\mathrm{x}): \quad[18] 1-$ 136, 1-144.

$\mathrm{Ch}(\mathrm{x})=(\sin \mathrm{ht} / \mathrm{ht}) \mathrm{e}^{\mathrm{ait}}: \quad$ [1]259.

Special case of Type II: [2]142. 
$\mathrm{Ch}(\mathrm{x})$, bibliography for rectangular over (a,b), (-a, a): [17] Nos. 11, 12.

\section{$\mathrm{C}(\mathrm{x})=\frac{1}{2}+\mathrm{x} / \mathrm{a}: \quad[15] 93$.}

If $\mathrm{x}$ and $\mathrm{y}$ are the $\mathrm{k}^{\text {th }}$ values from the top and bottom of sample,

$\mathrm{E}(\mathrm{x})=\mathrm{a}+\mathrm{h}-\frac{\mathrm{k}}{\mathrm{n}+1}(2 \mathrm{~h}) ; \quad \mathrm{E}\left[\frac{1}{2}(\mathrm{x}+\mathrm{y})\right]=\mathrm{a} ;$

$$
\mathrm{E}(\mathrm{x}-\mathrm{y})=\left(1-\frac{2 \mathrm{k}}{\mathrm{n}+1}\right)(2 \mathrm{~h}): \quad \text { [1]372. }
$$

$\operatorname{Var}(\mathrm{x})=\frac{\mathrm{k}(\mathrm{n}-\mathrm{k}+1)}{(\mathrm{n}+1)^{2}(\mathrm{n}+2)}(2 \mathrm{~h})^{2}$

$\operatorname{Var}\left[\frac{1}{2}(x+y)\right]=\frac{4 k h^{2}}{2(n+1)(n+2)}$

$\operatorname{Var}(\mathrm{x}-\mathrm{y})=\frac{2 \mathrm{k}(\mathrm{n}-2 \mathrm{k}+1)}{(\mathrm{n}+1)^{2}(\mathrm{n}+2)}(2 \mathrm{~h})^{2}$

$\sim \mathrm{D}(\overline{\mathrm{x}})=\mathrm{N}\left(0, \frac{\mathrm{h}^{2}}{3 \mathrm{n}}\right), \sim \mathrm{D}(\mathrm{c})=$ Laplace $\left(0, \frac{\mathrm{h}}{\mathrm{n}}\right)$,

where $c=\frac{1}{2}(\max +\min ), \mathrm{n} \operatorname{var}(\mathrm{c})=6 \operatorname{var}(\mathrm{x})$ :

$\mathbf{D}(\xi): \quad[d] 26: 115$.

$[3] 48$.

Moments of max and min:

$\operatorname{MR~4:21.~}$

$D(\overline{\mathrm{x}})$ given incorrectly: $\quad[n] 10-3: 91$.

$D(\overline{\mathrm{x}})=$ No. 5.16 .

$\mathrm{D}\left(-2 \log \Pi \mathrm{x}_{\mathrm{j}}\right): \quad[v]_{4}: 161$.

$\mathrm{FD}(\mathrm{a})=\operatorname{Rectangular}(\max -\mathrm{h}, \min +\mathrm{h}): \quad[c] 30: 402$.

$\mathrm{D}\left(\mathrm{q}^{\text {th }}\right.$ ranking item $)=$ Type $\mathrm{I}: \quad[c] 23: 390$.

Testing against simple unimodal distribution: $[y] 20: 111$.

Grouping corrections: $\quad[c] 39: 430$.

Transformation to Cauchy: [15]101.

C.-R. theorem may not hold: [1]485.

$\operatorname{MLE}(\mathrm{a}-\mathrm{h}, \mathrm{a}+\mathrm{h})=(\max , \min ): \quad[6] 156,[3] 28$.

Best linear estimate of $\mathrm{m}$ and $\sigma: \quad$ [d]25:308,31\%.

Estimation: $[d] 17: 355$.

Location and scaling, closest estimate: $[u] 33: 221$.

Minimax estimate of "a": [d]22:37.

UMVUE: [14]142.

Bayes' theorem: $[n] 16-1: 110$.

Variance of estimates of "a": $[\mathrm{g}] 36: 410$.

Testing "a": [d]25:15\%.

Critical regions: [3]280.

See also: Archiv. der Math. 3:3, MR 6:235.

\subsection{Rectangular $(0, a)$}

$\mathrm{D}(\mathrm{x})=1 / \mathrm{a}, \mathrm{Ch}(\mathrm{x})=\left(\mathrm{e}^{\mathrm{ait}}-1\right) / \mathrm{ait}: \quad$ [2]245 .

$\operatorname{MGF}(\mathrm{x})=\left(\sinh \frac{1}{2} \mathrm{at}\right) / \frac{1}{2} \mathrm{at}: \quad[10] 38$. $\alpha_{2 \mathrm{r}}=\left(\frac{1}{2} \mathrm{a}\right)^{2 \mathrm{r}} /(2 \mathrm{r}+1): \quad[10] 14$.

Cumulants: $\quad[10] 41$.

$\operatorname{Var}($ mean deviation $) \simeq \mathrm{a}^{2} / 45 \mathrm{n}: \quad$ [2]21\%.

Mean difference: $[c] 28: 432$.

$\mathrm{Ch}\left(\Sigma \mathrm{x}_{\mathrm{j}}\right), \mathrm{C}\left(\Sigma \mathrm{x}_{\mathrm{j}}\right): \quad[9] 278$.

$\mathrm{D}(\mathrm{GM})=\frac{\mathrm{n}^{\mathrm{n}} \mathrm{x}^{\mathrm{n}-1}}{a^{\mathrm{n}} \Gamma(\mathrm{n})}(\log \mathrm{a} / \mathrm{x})^{\mathrm{n}-1}: \quad$ [2]246,[d]5:276.

$\mathrm{D}($ range $)=\mathrm{n}(\mathrm{n}-1) \mathrm{a}^{-\mathrm{n}} \mathrm{X}^{\mathrm{n}-2}(\mathrm{a}-\mathrm{x}):$ [4]92, 123, [c]20A:210, [9]241.

$\mathrm{D}\left(\sum_{\mathrm{j}=1}^{\mathrm{n}} \log \mathrm{x}_{\mathrm{j}}\right)=$ Type III: $\quad[v] \%: 296$.

$\mathrm{D}(\overline{\mathrm{x}}, \mathrm{s})$ for $\mathrm{n}=2,3: \quad[d] 3: 128$.

$\mathrm{D}$ (quotient of ranges): $\quad[\mathrm{g}] 46: 502$.

$\mathrm{D}\left(\max _{1} / \max _{2}\right)=$ No. 8.63: $\quad[g] 50: 1136$.

$\mathrm{FD}(\mathrm{a})=\mathrm{k} \mathrm{x}^{-\mathrm{n}-1}: \quad[c] 30: 408,[d] 9: 273$.

$\operatorname{MGF}\left(\log \log \frac{1}{\mathrm{x}}\right)$, MGF $(\arcsin \sqrt{\mathrm{x}}): \quad[v] 8: 69$.

Completeness: $[e] 10: 314$.

Estimation by order statistics: $\quad[d] 26: 576$.

Quasi-range: $\quad[d] 28: 179$.

Best linear estimate: $[d] 14: 88$.

UMVUE $(\mathrm{a})=\left(1+\frac{1}{\mathrm{n}}\right)(\max ), \max$ is sufficient:

[14]142.

Sufficient statistics: $\quad[e] 17: 214$.

Confidence intervals for a: $[3] 83,[b] 17: 88$.

Example: $[d] 11: 209$.

Estimation of dispersion: [c]36:95.

UMP test of $\mathrm{a}=1: \quad[13]$.

See also: $[d] 2: 48,[d] 2: 66,[d] 4: 126,[d] 4: 139$, $142,255,[c] 23: 424$.

\subsection{Rectangular $(0,1)$}

$\mathrm{D}(\mathrm{x})=1, \quad 0 \leqq \mathrm{x} \leqq 1$

$\mathrm{D}(-\log \mathrm{x})=\mathrm{e}^{-x}, \operatorname{Ch}(-\log \mathrm{x})=(1-\mathrm{it})^{-1}$

$\mathrm{D}\left(-\Sigma \log \mathrm{x}_{\mathrm{j}}\right)=$ Type III $(\mathrm{l}, \mathrm{k}): \quad[3] 132$.

$\mathrm{D}\left(\Sigma \mathrm{x}_{\mathrm{j}}\right)=\frac{1}{(\mathrm{n}-1) !}\left[\mathrm{x}^{\mathrm{n}-1}-\left(\begin{array}{l}n \\ 1\end{array}\right)(\mathrm{x}-1)^{\mathrm{n}-1}\right.$

$$
\left.+\left(\begin{array}{c}
\mathrm{n} \\
2
\end{array}\right)(\mathrm{x}-2)^{\mathrm{n}-1}-\ldots\right]
$$

"Irwin-Hall" distribution: [d]13:43, [1]245, $[w]$ 1:73, [2]240, 244, [c]19:234, MR 12:509, MR 15:42, MR $7: 311,[c] 19: 240,[c] 41: 334$.

Obtained by finite differences (Also for No.4.4): $[p] 10: 52,255,324$.

Convolutions: $\quad M R$ 6:88.

Sheppard's corrections: [2] 88 . 
$\mathrm{D}\left(\mathrm{q}^{\text {th }}\right.$ value from top of sample $)=$ Type $\mathrm{I}$, $\mathrm{m}=1-\mathrm{q} /(\mathrm{n}+1), \mathrm{v}=\mathrm{q}(\mathrm{n}-\mathrm{q}+1) /\left[(\mathrm{n}+1)^{2}(\mathrm{n}+2)\right]$ : [2]218.

$\operatorname{Var}(\xi)=1 / 4(\mathrm{n}+2): \quad$ [2]230.

$\sim \mathrm{D}(\bar{x}): \quad[d] 25: 636, \operatorname{MR} 9: 360$.

Mellin transform: $\quad[d] 19: 373$.

Order statistics: $\quad[c] 24: 260,[i] 33: 214$.

$\mathrm{D}($ range $)=$ Type $\mathrm{I}: \quad[c] 25: 417$.

$\operatorname{MGF}(\log \log 1 / \mathrm{x})=(1+\mathrm{t})$

$\operatorname{MGF}(\arcsin \mathrm{x})=2\left(\mathrm{e}^{\frac{1}{2} \pi \mathrm{t}}+1\right) /\left(\mathrm{t}^{2}+4\right): \quad[v] 8: 69$.

Ratio of two ranges: $\quad[d] 21: 112,[x]^{7}: 179$.

Moments of the range: $\quad Z$ 13:30, [c]20:217.

C. - R. theorem may not hold: [1]485.

Censored sample: $[c] 41: 230$.

Stratified sample: $[d] 13: 44$.

Significance levels for $\overline{\mathrm{x}}: \quad[t] 3: 172$.

$\mathrm{D}(\mathrm{GM})=$ No. 8.12: $\quad[w] 1: 73$.

Estimation of center, $\mathrm{D}(\overline{\mathrm{x}}), \quad \mathrm{D}(\xi): \quad[c] 33: 126$.

$\mathrm{D}\left(\max _{1} \max _{2}\right)=$ No. 8.64: $[g] 50: 1142$.

Two rectangulars added: $[n] 8-3: \% 4$.

Hypothesis testing: [c]32:321.

See Also: $[d] 23: 43,[c] 25: 203,[m] 6: 120,[d] 22:$ 418, [y]24:21, MR 7:310, Z 11:218, MR 16:602.

5.14. Correlation

$\mathrm{D}(\mathrm{x})=\frac{\left(1-\mathrm{x}^{2}\right)^{\frac{1}{2}(\mathrm{n}-4)}\left(1-\rho^{2}\right)^{\frac{1}{2}(\mathrm{n}-1)} 2^{\mathrm{n}-3}}{\pi(n-3) !}$ $\sum_{\mathrm{i}=0}^{\infty} \frac{(2 \mathrm{x} \rho)^{\mathrm{i}}}{\mathrm{i} !} \Gamma^{2}\left(\frac{\mathrm{n}+\mathrm{i}-1}{2}\right) \quad$ [1]398.

$\mathrm{D}(\mathrm{x})=\frac{\left(1-\rho^{2}\right)^{\frac{1}{2}(\mathrm{n}-1)}\left(1-\mathrm{x}^{2}\right)^{\frac{1}{2}(\mathrm{n}-4)}}{\pi(\mathrm{n}-2) !} \frac{\mathrm{d}^{\mathrm{n}-2}}{\mathrm{~d}(\rho \mathrm{x})^{\mathrm{n}-2}}\left[\frac{\cos ^{-1}(-\rho \mathrm{x})}{1-\rho^{2} \mathrm{x}^{2}}\right]$ [2]34ุ2, [10]200.

Special cases $\mathrm{n}=2,3,4$, moments: [2]345. $\mathrm{n}=4: \quad[u] 26: 536$.

$\mathrm{C}(\mathrm{x}): \quad[c] 25: 71$.

$\sim \mathrm{D}(\mathrm{x}): \quad[n] 1-4: 1,[c] 38: 236$.

If $\rho=0, \quad \mathrm{D}(\mathrm{x})=$ No. 5.5: $\quad[6] 314,[4] 120,[g] 26: 129$.

If $\rho=0, \quad D\left(\frac{x}{\sqrt{1-x^{2}}} \sqrt{n-2}\right)=\operatorname{Student}(n-2)$ :

[2]343.

Transform $\mathrm{x}=\tanh \mathrm{z}, \rho=\tanh \zeta: \quad[10] 200,[c] 21$ : 358.

If $\rho=0, \mathrm{D}\left(\mathrm{x}^{2}\right)=\operatorname{Beta}\left[\frac{1}{4}, \frac{1}{4}(\mathrm{n}-2)\right]: \quad[10] 160,192$.

Bayes' distribution of $\rho$ is No. 5.5 $(0,1)$ : [3]91, $[c] 41: 278$.

Moments: $[n] 5: 3$.
Papers dealing with this distribution generally: $[c] 10: 507,[c] 11: 328$.

Interval estimation: $[e]^{7}: 415$.

Confidence limits for $\rho$ : $\quad[c] 29: 15 \%$.

Stratified sampling: $\quad[i] 36($ Suppl. $): 87$.

See also: $\quad[b] 15: 193,[c] 21: 164,[c] 24: 383 ;[0] 3: 1$, Z21:41.

\subsection{Multiple Correlation}

$\mathrm{D}(\mathrm{x})=\frac{\gamma\left(1-\mathrm{R}^{2}\right)^{\frac{1}{2}(\mathrm{n}-1)}}{\mathrm{B}\left[\frac{1}{2}(\mathrm{n}-\mathrm{k}),(\mathrm{k}-1)\right]^{\mathrm{x}^{\frac{1}{2}(\mathrm{k}-3)}}(1-\mathrm{x})^{\frac{1}{2}(\mathrm{n}-\mathrm{k}-2)}}$

where $\gamma=F\left(\frac{n-1}{2}, \frac{n-1}{2}, \frac{k-1}{2}, R^{2} x\right)$ and

$\mathrm{F}(\mathrm{a}, \mathrm{b}, \mathrm{c}, \mathrm{x})=1+\frac{\mathrm{ab}}{\mathrm{c}} \mathrm{x}+\frac{\mathrm{a}(\mathrm{a}+1) \mathrm{b}(\mathrm{b}+1)}{\mathrm{c}(\mathrm{c}+1)} \frac{\mathrm{x}^{2}}{2 !}+\cdots \cdot$

[14]65, [2]384, [d]3:196, [2]127.

If $\mathrm{R}=0, \mathrm{D}\left(\mathrm{x}^{2}\right)=\operatorname{Beta}(\mathrm{k}, \mathrm{n}-\mathrm{k}): \quad$ [i]30:63, [2]381

Testing: [i] 29:25

Another form: [2]38\%, [3]338.

Limiting form when $\mathrm{n} \rightarrow \infty$ : [2]38\%.

When $\mathrm{R}=0, \mathrm{D}(\mathrm{x})=$ Snedecor: $\quad[10] 257,252$.

Mean, variance: [c]22:353.

Moments: $\quad$ MR 14:189.

More generally: $\quad[d] 11: 6$.

See also: [e]9:352, [e]10:257, [a]92:445, [n]124:67, [i] 24:199, [x]1:67,137, [x]4:88, Proc. Roy. Soc. (A) $121: 654,[u] 46: 521$.

\subsection{Rectangular Mean}

$\mathrm{D}(\mathrm{x})=\frac{1}{(\mathrm{n}-1) !} \sum_{\mathrm{j}=0}^{[\mathrm{x}]}(-1)^{\mathrm{j}}\left(\begin{array}{l}\mathrm{n} \\ \mathrm{j}\end{array}\right)(\mathrm{x}-\mathrm{j})^{\mathrm{n}-1}: \quad[1] 245$.

Generalization: $[v] 3: 330$.

Obtained by finite differences: $\quad[p] 10: 52,255,324$.

Called Irwin-Hall, ef. No. 5.13 [d]13:43.

Compare No. 8.47 and No. 8.70:

See also: London P.O. Res. Rep. 13443, Archiv der Math. 3:3, Proc. Intl. Cong. Math. (1924) 2:795.

\section{Distributions on $(0, \infty)$}

\subsection{Type VI}

$\mathrm{D}(\mathrm{x})=\mathrm{C}(\mathrm{x}-\mathrm{a})^{\mathrm{p}-1}(\mathrm{x}-\mathrm{b})^{\mathrm{q}-1}, \quad \mathrm{x}>\mathrm{b}, \quad \mathrm{a}<\mathrm{b}, \quad \mathrm{q}>0$, $\mathrm{p}+\mathrm{q}<1: \quad[1] 249$.

If $\mathrm{b}=0, \quad \mathrm{C}=\frac{\mathrm{a}^{1-\mathrm{p}-\mathrm{q}}}{\mathrm{B}(1-\mathrm{p}-\mathrm{q}, \mathrm{p})}: \quad$ [2]140.

Roots of quadratic in Pearson equation real and same sign: $\quad[11] 45$. 
Truncation: $\quad[i] 39: 63,[i] 40: 18$.

Various constants and an example: [11]83.

See also: $\quad[d]$ 7\%:23, $[c] 23: 143,[c] 25: 379,[17]$ No. $26,[v] 4: 167$.

\subsection{Snedecor $(p, q)$}

$\mathrm{D}(\mathrm{x})=\frac{(\mathrm{p} / \mathrm{q})^{\frac{1}{2} \mathrm{p}} \mathrm{X}^{\frac{1}{2} \mathrm{p}-1}(1+\mathrm{px} / \mathrm{q})^{-\frac{1}{2}(\mathrm{p}+\mathrm{q})}}{\mathrm{B}\left(\frac{1}{2} \mathrm{p}, \frac{1}{2} \mathrm{q}\right)}, \mathrm{x}>0: \quad[5] 100$.

"F" distribution

$\mathrm{m}, \mathrm{v}, \kappa_{3}, \kappa_{4}, \beta_{1}, \beta_{2}: \quad[p] 6: 175$.

Derivation, properties, examples: [15]3\%4, [18]1-163.

Area unity if p,q both even: MR 12:509.

Obtained as distribution of ratio of two Chi-square variables: $[6] 10.5,[4] 113$.

$1-\mathrm{C}(\mathrm{x})=\frac{\sum_{0}^{\mathrm{n}} \mathrm{c}_{\mathrm{j}} \mathrm{x}^{\mathrm{j}}}{(\mathrm{x}+\mathrm{k})^{\mathrm{m}}}, \quad$ where $\mathrm{n}=\frac{1}{2} \mathrm{p}-1$,

$\mathrm{m}=\frac{1}{2}(\mathrm{p}+\mathrm{q})-1, \mathrm{k}=\mathrm{q} / \mathrm{p}$, and $\mathrm{c}_{\mathrm{j}}=\left({ }_{\mathrm{j}}\right) \mathrm{k}^{\mathrm{m}-\mathrm{j}}$ :

$[p] 10: 62$.

$\mathrm{D}(\sqrt{\mathrm{x}}): \quad[5] 100$.

$\mathrm{D}\left(\frac{\mathrm{px}}{\mathrm{q}+\mathrm{px}}\right)=\mathrm{B}(\mathrm{p}-1, \quad \mathrm{q}-1): \quad$ [4]115

Therefore called "inverted Beta": [c]33:73.

Various properties: $\quad[d] 12: 446$.

$\mathrm{m}=\frac{\mathrm{q}}{\mathrm{q}-2}: \quad[10] 198$.

$\alpha_{\mathrm{r}}=\frac{\Gamma\left(\frac{1}{2} \mathrm{p}+\mathrm{r}\right) \Gamma\left(\frac{1}{2} \mathrm{q}-\mathrm{r}\right)}{\Gamma\left(\frac{1}{2} \mathrm{p}\right) \Gamma\left(\frac{1}{2} \mathrm{q}\right)}(\mathrm{q} / \mathrm{p})^{\mathrm{r}}: \quad[4] 114$.

Mode $=\frac{p q-2 q}{p q+2 p}: \quad[10] 197$.

Approximated by normal distribution: [d]13:233. If $x, y$ each Snedecor $(n-1, n)$, then $\mathrm{D}(\sqrt{\mathrm{x} / \mathrm{y}})=$ Snedecor $(2 \mathrm{n}-2,2 \mathrm{n}-2)$.

Testing: $\quad[d] 13: 371$.

Used to test multiple correlation coefficient:

$[10] 25 \%$.

See also: [4]189, [d]6:204, [d]18:89, [c]21:350, $[c] 37: 219,[q]^{77}: 96$, J. Soc. Statist. Paris 96:262.

6.3. Beta of Second Kind (p, q)

$\mathrm{D}(\mathrm{x})=\frac{\mathrm{x}^{p-1}}{\mathrm{~B}(\mathrm{p}, \mathrm{q})(1+\mathrm{x})^{\mathrm{p}+\mathrm{q}}}, \quad \mathrm{m}=\frac{\mathrm{p}}{\mathrm{q}-1}$,

$\mathrm{v}=\frac{\mathrm{p}(\mathrm{p}+\mathrm{q}-1)}{(\mathrm{q}-1)^{2}(\mathrm{q}-2)}, \operatorname{mode}=\frac{\mathrm{p}-1}{\mathrm{q}+1}, \mathrm{HM}=\frac{\mathrm{p}-1}{\mathrm{q}}$ if $r<q, \alpha_{r}=\frac{p(p+1) \ldots(p+r-1)}{(q-1)(q-2) \ldots(q-r)}$

$\mathrm{D}(1 / \mathrm{x})=$ No. 6.3 $(\mathrm{q}, \mathrm{p}), \mathrm{D}\left(\frac{1}{1+\mathrm{x}}\right)=$ Beta:

[1]242, [10]156, 158, $163[d] 25: 402$.

$\mathrm{p}=\mathrm{q}, \mathrm{x} \geqq 1: \quad[d] 22: 418$.

Called Fisher's F: $\quad[w] 1: 9$.

$\mathrm{C}(\mathrm{x}): \quad[p]^{\gamma} \%: 102$.

\subsection{Hotelling}

$\mathrm{D}(\mathrm{x})=\frac{2}{\mathrm{~B}\left[\frac{1}{2}(\mathrm{p}-\mathrm{q}), \frac{1}{2} \mathrm{q}\right](\mathrm{p}-1)^{\frac{1}{2} \mathrm{q}}}\left[\frac{\mathrm{x}^{\mathrm{q}-1}}{\left[1+\frac{\mathrm{x}^{2}}{\mathrm{p}-1}\right]^{\frac{1}{2} \mathrm{p}}}: \quad\right.$ [4]238.

For $q=1$, this is positive half of Student distribution, hence called generalized Student:

$[1] 409,[d] 2: 375$.

$\mathrm{D}\left(\mathrm{x}^{2}\right): \quad[c] 32: 70$.

Mellin transform: $[d] 19: 373$.

Percentage points, relation with Chi-square:

See also: $[10] 20 \%,[c] 25: 399,[i] 30: 66,[d] 9: 235$. $[c] 24: 480,[c] 4: 174,[c] 24: 487,[t] 7: 82$.

\subsection{Pareto}

$\mathrm{D}(\mathrm{x})=\mathrm{p} / \mathrm{q}(\mathrm{q} / \mathrm{x})^{\mathrm{p}+1}: \quad[8] 120$.

$\alpha_{1}=\frac{\mathrm{p}}{\mathrm{p}-1} \mathrm{q}, \xi=2^{1 / \mathrm{p}} \mathrm{q}: \quad[1] 248,[2] 142$.

More generally: $[d]^{y}: 26$.

Testing, location and dispersion: $[t]^{7}: 115$.

As Type XI: $\quad[c] 39: 178,[17]$ No. 25.

Ranking: $\quad[c] 24: 234,241,275$.

Double Pareto: Kendall and Buckland, A Dictionary of Stat. Terms.

See also: $[l] 19: 1 \% 4,\left[h^{\prime}\right] 1: 149,[g] 48: 53 \%,[i] 8: \% 6$, [t]3:77, [y]13:30, MR13:962, Z23:63, C. R. Acad. Sci. Paris 233:1421, [l]25:591, [w] 4:14\%.

\subsection{Kendall}

$\mathrm{D}(\mathrm{x})=\frac{\mathrm{re}^{-(\mathrm{x}-\mathrm{r}) / \alpha}(\mathrm{x}-\mathrm{r})^{\mathrm{x}-1}}{\alpha^{x} \Gamma(\mathrm{x}+1)}, \quad 0<\mathrm{r}<\mathrm{x}<\infty, \quad 0 \leq \alpha \leq 1$, $\mathrm{m}=\frac{\mathrm{r}}{1-\alpha}, \mathrm{v}=\frac{\mathrm{r} \alpha^{2}}{(1-\alpha)^{3}}$.

[b]19:211, (cf. Borel-Tanner).

\subsection{Inverse Gaussian}

$\mathrm{D}(\mathrm{x})=\exp \quad\left[-\lambda(\mathrm{x}-\mu)^{2} / 2 \mu^{2} \mathrm{x}\right]\left[\lambda / 2 \pi \mathrm{x}^{3}\right]^{\frac{1}{2}}, \quad$ introduction, moments, estimation: $[d] 28: 362,696$. 


\section{Distributions on $(-\infty, \infty)$}

\subsection{Type VII}

$\mathrm{D}(\mathrm{x})=\frac{\left(1+\mathrm{x}^{2} / \mathrm{a}^{2}\right)^{-\mathrm{m}}}{\mathrm{aB}\left(\frac{1}{2}, \mathrm{~m}-\frac{1}{2}\right)}, \quad \mathrm{m}>\frac{1}{2}: \quad$ [2]142.

Estimation: $\quad[c] 36: 412,[t] 4: 35$.

See also: $\quad[c] 15: 401,[c] 36: 412,167$.

\subsection{Student $(\nu)$}

$\mathrm{D}(\mathrm{x})=\frac{\Gamma\left[\frac{1}{2}(\nu+1)\right]}{\Gamma\left(\frac{1}{2} \nu\right)(\pi \nu)^{\frac{1}{2}}}\left(1+\mathrm{x}^{2} / \nu\right)^{-\frac{1}{2}(\nu+1)}:$

$[5] 97,[2] 17,[6]$

10.6, MR8:161, [14]47, [4]110, [10]186, [l]3:355, [18]1-162, [17]No. 29.

Introduction, properties, examples: [15]388.

$\checkmark=\nu^{\prime}(\nu-2) . \quad[1] 239$.

Type VII with $\mathrm{m}=\frac{1}{2}(\nu+1), \mathrm{a}^{2}=\nu$.

"t" distribution.

$\alpha_{2 \mathrm{k}}=\frac{1 \cdot 3 \cdot \ldots \cdot(2 \mathrm{k}-1) \nu^{\mathrm{k}}}{(\nu-2)(\nu-4) \cdot \ldots \cdot(\nu-2 \mathrm{k})}: \quad$ [1]239, [10]208.

Original paper in which this distribution was discovered: $\quad[c] 6: 1$.

$\mathrm{Ch}(\mathrm{x})$, refs for $\nu=3: \quad[17]$ No. 28 .

$\mathrm{Ch}(\mathrm{x}): \quad[b] 18: 212$.

Distribution of the ratio of a Chi-square variable to a normal variable: [1]387, [4]110, [10]187 [n]5: 102.

In bivariate normal samples

$$
\begin{aligned}
& \mathrm{D}\left(\frac{\sigma_{1}(\mathrm{n}-1)^{\frac{1}{2}}}{\sigma_{2}\left(1-\rho^{2}\right)^{\frac{1}{2}}}(\mathrm{~b}-\beta)\right)=\text { Student }(\mathrm{n}-1): \quad \text { [1]29.8. } \\
& \mathrm{D}\left(\frac{\mathrm{s}_{1}(\mathrm{n}-2)^{\frac{1}{2}}}{\mathrm{~s}_{2}\left(1-\nu^{2}\right)^{\frac{1}{2}}}(\mathrm{~b}-\beta)\right)=\text { Student }(\mathrm{n}-2):
\end{aligned}
$$

if $\rho=0$

$$
\mathrm{D}\left[(\mathrm{n}-2)^{\frac{1}{2}} \frac{\nu}{\left(1-\nu^{2}\right)^{\frac{1}{2}}}\right]=\text { Student }(\mathrm{n}-2): \quad[1] 29.7 .
$$

$\mathrm{C}(\mathrm{x}): \quad[l] 3: 358,[c] 25: 389,[n] 5: 109,[c] 37: 168$.

\section{$\mathrm{D}\left(\mathrm{x}^{2}\right)=$ Snedecor: $\quad[6] 217,[4] 115$.}

Transform to Type II: [c]28:308.

$\mathrm{D}(\overline{\mathrm{x}}): \quad[n] 8-4: 92$.

As $\nu \rightarrow \infty$, Student $\rightarrow \mathrm{N}(0,1): \quad$ [1]252, [3]101, [a] 113:228, [d]27:783, Proc. A.M.S. 6th Symposium in Appl. Math. p. 251.

Approximations: $[d] \%: 210,[d] 9: 87,[d] 17: 216$.

$\mathrm{D}(\log \mathrm{x}): \quad[c] 34: 176$.

Two Student variables: $[c] 22: 405,[c] 23: 1$.

Used to test partial correlation: [10]256.

$\sim$ significance levels: $\quad[d] 14: 60$.
Generalizations: $\quad[d] 19: 406,[d] 25: 162,[i] 34: 58$.

See also: $\quad[d] 10: 265,[d] 18: 89,[e] 11: 37,[e] 12: 89$, [j]8:632, [c]33:362, [n]5:90, [c]32:271,300, [c]24:56,296, [i]33:138, [c]44:264, Brit. Assoc. Math. Tables (3rd Ed.) V. 1 p xxxiii, J. Soc. Stat. Paris 92:262, MR18: $834, Z_{4}: 67,[u] 21: 482,655,[w] 1: 9,[p] 8: 42$.

\subsection{Normal Regression Slope}

$\mathrm{D}(\mathrm{x})=\frac{\left[\sigma_{1}^{2} \sigma_{2}^{2}\left(1-\rho^{2}\right)\right]^{\frac{1}{2}(\mathrm{n}-1)} \Gamma\left(\frac{1}{2} \mathrm{n}\right)}{\sqrt{\pi} \Gamma\left[\frac{1}{2}(\mathrm{n}-1)\right] \sigma_{1}^{\mathrm{n}-2}\left(\sigma_{2}^{2}-2 \rho \sigma_{1} \sigma_{2} \mathrm{X}+\sigma_{1}^{2} \mathrm{x}^{2}\right)^{\frac{1}{2}} \mathrm{n}}:$

[1]402.

$\mathrm{v}=\frac{1}{\mathrm{n}-3} \frac{\sigma_{2}^{2}}{\sigma_{1}^{2}}\left(1-\rho^{2}\right): \quad[2] 365,[e] 1: 432$.

For $\rho=0$, can use Student distribution to test $\mathrm{x}$ : $[10] 194$.

Stratified sampling: $\quad[i] 36: 96$.

7.4. Cauchy $(\mathrm{p}, \mathrm{q})$

$\mathrm{D}(\mathrm{x})=\frac{1}{\pi} \frac{\mathrm{p}}{\mathrm{p}^{2}+(\mathrm{x}-\mathrm{q})^{2}}: \quad[1] 246,[5] 35,[18] 1-36$.

$\mathrm{Ch}(\mathrm{x})=\exp [q i t-\mathrm{p}|\mathrm{t}|]: \quad[5] 60$.

$q$ is the mode and median; there is no mean, nor any moment: [5]58.

Quartiles are $\mathrm{q} \pm \mathrm{p}: \quad[5] 67$.

$\overline{\mathrm{x}}$ is not a consistent estimate of $q$ : [5]105.

$\overline{\mathrm{x}}$ is a "density unbiased" estimate of q: $\quad[d] 25: 400$.

There are no sufficient estimators: [3]48.

C. -R. (q), C. -R. (p), C. -R. (p/q): [e]8:205.

Distribution of $\mathrm{t}$ and $\mathrm{F}$ statistics: MR13:665.

Mean and variance of $\frac{1}{2}(x+y)$, where $x$ and $y$ are respectively the $\mathrm{k}^{\text {th }}$ values from the top and bottom of the sample: [1]373. $\frac{1}{2}(x+y)$ is not a consistent estimate of $q$.

See also: $\quad[d] 17: 2,[d] 21: 133$

7.5. Cauchy $(1, q)$

$\mathrm{D}(\mathrm{x})=\frac{1}{\pi} \frac{1}{1+(\mathrm{x}-\mathrm{q})^{2}}: \quad[6] 117$.

$q$ incorrectly asserted to be the mean: $[p]$ \%:165.

$\mathrm{E}(\mathrm{x}), \mathrm{E}\left(\mathrm{x}^{2}\right), \mathrm{D}(\mathrm{x}+\mathrm{y}): \quad[14] 43$.

C. - R. $(\mathrm{q})=2 / \mathrm{n}: \quad[1] 490,[3] 24,[p]^{7}: 159$.

$\operatorname{Var}(\xi) \cong \pi^{2} / 4 \mathrm{n}: \quad[3] 6$.

$\mathrm{D}(\overline{\mathrm{x}})=\mathrm{D}(\mathrm{x})$, hence $\overline{\mathrm{x}}$ not consistent: $\quad[3] 2,[1] 490$, [u]22:702.

$\mathrm{D}(\xi): \quad[3] 46$.

MLE $\neq$ minimax: $\quad[16] 64$.

MLE is solution of $\Sigma\left[2\left(x_{1}-q\right)\right] /\left[1+\left(x_{1}-q\right)^{2}\right]=0$ : $[3] 24,[p] 7: 169$.

Gaging: [e]15:194. 
There is no sufficient estimator: $\quad[9] 6: 16,[3] 2 \%$, $[p]^{7}: 162$.

There is no UMVUE: [3]51.

Information and estimation: [e]8:315.

Loss of information: [3]32.

Testing $\mathrm{m}=\mathrm{m}_{0}: \quad[d] 9: 83,[d] 13: 65$.

Cauchys added: MR17:863.

See also: $\quad[b] 9: 61,[i] 20: 61,[g] 51: 641,[d] 28: 832$, $[w] 1: 9$.

7.6. Cauchy $(p, 0)$

$\mathrm{D}(\mathrm{x})=(\mathrm{p} / \pi)\left[1 /\left(\mathrm{p}^{2}+\mathrm{x}^{2}\right)\right], \quad \mathrm{Ch}(\mathrm{x})=\exp -\mathrm{p}|\mathrm{t}|: \quad[9] 275$.

Reproductive property: [9]276.

Information and estimation: [e]8:316.

Completeness: $[e] 10: 314$.

$\mathrm{D}(\overline{\mathrm{x}})=\mathrm{D}(\mathrm{x}): \quad[n] 10-3: 91$.

Truncated to $(-\mathrm{p}, \mathrm{p}): \quad[10] 14$.

7.7. Cauchy $(1,0)$

$\mathrm{D}(\mathrm{x})=(1 / \pi)\left[1 /\left(1+\mathrm{x}^{2}\right)\right] \quad[8] 16 \%$.

$\mathrm{Ch}(\mathrm{x})=\exp -|\mathrm{t}|: \quad$ [2]95, [8]167, [1]246, [9]243, [17] No. 27, [s]213:718, [i]5:133.

Sample median: $\quad[d] 26: 600$.

$\mathrm{D}(\overline{\mathrm{x}})=\mathbf{D}(\mathrm{x}): \quad[2] 233,247,[w] 1: 73$.

From an example: [8]33, [9]242.

Moments: [8]99.

As distribution of ratio of two normal variables: [10]159.

$\mathrm{C}(\mathrm{x})=\frac{1}{2}+(1 / \pi) \tan ^{-1} \mathrm{x}$.

Censored sample: $[c] 41: 230$.

Wrapped-up Cauchy: $\quad[d] 26: 245$.

See also: $\quad[d] 22: 425,[k]^{7}: 371$, S. D. Poisson (1824) "Sur la Probabilité des résultats moyens des observations," Connaissance des Tems ou Des Mouvements Célestes a l'usage des astronomes et des Navigateurs, pour l'an 182\%. Le Bureau des Longitudes, Paris. [d]22:418, MR3:232, MR9:235, MR5:124.

7.8. LaPlace $(\mathrm{m}, \mathrm{v})$

$\mathrm{D}(\mathrm{x})=(2 \mathrm{v})^{-\frac{1}{2}} \exp \left[-|\mathrm{x}-\mathrm{m}| / \frac{1}{2}(2 \mathrm{v})^{\frac{1}{2}}\right]: \quad[1] 24^{7},[5] 35$, $67,[18] 1-136$.

$\mathrm{Ch}(\mathrm{x})=\exp (\mathrm{mit}) \cdot\left(1+\frac{1}{2} \mathrm{vt}^{2}\right)^{-1}: \quad$ [5]62.

Mean and variance of average of greatest and least sample values: $\quad[1] 375$.

A priori distributions of $\mathrm{m}, \mathrm{v}$ : MR9:294.

"Best" estimates of $\mathrm{m}$ and $\sigma$ are $\xi$ and $(1 / \mathrm{n}) \Sigma\left|\mathrm{x}_{1}-\xi\right|$, but $\xi$ not sufficient: $[5] 147-8$.

Distribution of smallest sample value: $[\mathrm{g}] \mathrm{43}: 408$.

$\mathrm{D}(\xi): \quad[d] 26: 115$.
Quasi-range: $\quad[d] 28: 179$.

"Double exponential" distribution: Proc. Roy.

Soc. Lond. (Series A) 154:124, [v]\%:164.

Convolution, estimation, generalization: Sobre la Primera Ley de Errores de Laplace, F. A. Sales Vallés, Thesis, Barcelona 194\%.

Laplace $(0, \mathrm{v})$, also called "Poisson's first law of error," D $(|\bar{x}|), \quad D(|x|-|y|), \quad D(\log |x|-\log |y|)$, $\mathrm{D}\left(\Sigma \mathrm{x}_{1}^{2}\right), \mathrm{D}(\mathrm{GM}), \mathrm{D}(\mathrm{HM}): \quad[d] 6: 102$.

Laplace if $\mathrm{D}(\mathrm{x}) \mathrm{D}(\mathrm{y})=\phi(|\mathrm{x}|+|\mathrm{y}|) \quad$ MR10:125.

See also: $\quad[n] 10-3: 80$.

Laplace $(\mathrm{m}, 2)$, MLE: $\quad[3] 45$.

Laplace $(0,1), \mathrm{D}(\mathrm{x})=\mathrm{e}^{-|\mathrm{x}|}: \quad[8] 120,[9] 279,[1] 100$.

Sample median: $\quad[d] 26: 599$.

$\alpha_{2 \mathrm{k}}=(2 \mathrm{k}) !: \quad[d] 5: 32$.

$\mathrm{D}$ (range), quality control: $[p] 8: 87$.

See also: [d]22:425, [17] No. 38, Laplace (1774), "Memoire sur la Probabilité des causes par les évènemens."

7.9. Fisher (p, q)

$\mathrm{D}(\mathrm{x})=\frac{2 \mathrm{p}^{\frac{1}{\mathrm{p}} \mathrm{p}} \mathrm{q}^{\frac{1}{2} \mathrm{q}} \mathrm{e}^{\mathrm{px}}}{\mathrm{B}\left(\frac{1}{2} \mathrm{p}, \frac{1}{2} \mathrm{q}\right)\left(\mathrm{q}+\mathrm{pe}^{2 \mathrm{x}}\right)^{\frac{1}{2}(\mathrm{p}+\mathrm{q})}}: \quad$ [1]243, [2]249,

B $\left(\frac{1}{2} \mathrm{p}, \frac{1}{2} \mathrm{q}\right)\left(\mathrm{q}+\mathrm{pe}^{2 \mathrm{x}}\right)^{\frac{1}{2}(\mathrm{p}+\mathrm{q})} \quad$ [14]48, MR8:161.

z distribution: [l]3:355.

$\mathrm{C}(\mathrm{x})$ as a power series: $[l] 3: 360$.

Cumulants: MR9:48, 735.

Moments, cumulants: $[y] 5: 31 \%$.

Transform to Snedecor by $\mathrm{y}=\mathrm{e}^{2 \mathrm{x}}$; $\mathrm{x}=\frac{1}{2} \log \mathrm{q} \chi_{1}^{2} / \mathrm{p} \chi_{2}^{2}: \quad[c] 23: 14 \%$.

Various properties: $[d] 12: 429,[c] 34: 173$.

$\mathrm{Ch}(\mathrm{x})=\left(\frac{\mathrm{q}}{\mathrm{p}}\right)^{\frac{1}{2} \mathrm{t} t} \Gamma\left[\frac{1}{2}(\mathrm{q}-\mathrm{it})\right] \Gamma\left[\frac{1}{2}(\mathrm{p}+\mathrm{it})\right] / \Gamma\left(\frac{1}{2} \mathrm{p}\right) \Gamma\left(\frac{1}{2} \mathrm{q}\right):$

$\sim \mathrm{C}(\mathrm{x}): \quad[d] 28: 504$.

[3]116.

Obtained from two Chi-square variables: [2]249, $[d]^{7}: 52,[g] 26: 173$.

Approximate significance levels, transformation to normal: $\quad[d] 11: 93$.

Normal limit: Am. Math. Monthly, 50:100.

Generalization: $[i] 34: 58$.

Noncentral: $[0] \%: 5 \%$.

See also: $[e] 2: 423,[b] 1: 31,[a] 94: 284,[c] 21: 350$, (c) 34:352, 359, [c]41:304, Am. Math. Monthly 50:100, 382. Proc. Intl. Cong. Math. (1920)805, Current Science 1941, p. 191, [w]5:30, [p]6:183.

\subsection{Type IV}

$\mathrm{D}(\mathrm{x})=\mathrm{C}\left(1+\mathrm{x}^{2} / \mathrm{a}^{2}\right)^{-\mathrm{m}} \exp \left(-\mathrm{p} \tan ^{-1} \mathrm{x} / \mathrm{a}\right):$

$\mathrm{D}(\mathrm{x}-\mathrm{h})=$ Type IV : $\quad[3] 48$.

[11]69. 
Various constants with an example: [11]69.

Roots of quadratic in Pearson equation complex:

$[11] 44$.

$\alpha_{\mathrm{r}}=[\mathrm{a} /(2 \mathrm{~m}-\mathrm{r}-1)]\left[(\mathrm{r}-1) \mathrm{a} \alpha_{\mathrm{r}-2}-\mathrm{p} \alpha_{\mathrm{r}-1}\right]: \quad$ [2]86, 140, 144.

$\mathrm{a}=1: \quad[17]$ No. 52.

See also: $[d] 7: 21,[c] 1: 39,[c] 3: 312,[c] 6: 435$, $[c] 7: 74,[c] 26: 386$.

\section{Miscellaneous Univariate}

\subsection{Pearson}

$\frac{1}{\mathrm{D}(\mathrm{x})} \frac{\mathrm{dD}(\mathrm{x})}{\mathrm{dx}}=\frac{\mathrm{a}_{0}+\mathrm{a}_{1} \mathrm{x}}{\mathrm{b}_{0}+\mathrm{b}_{1} \mathrm{x}+\mathrm{b}_{2} \mathrm{x}^{2}}$,

types listed with associated parameters: $[d] 2: 394$, $[n] 11-4: 7 \%$.

Differential equation for $\mathrm{Ch}(\mathrm{x})$ : MR8:393.

$\mathrm{Ch}(\mathrm{x}): \quad$ MR10:705.

Original paper: Phil. Trans., 1895.

First seven types treated in [11].

New classification: $\quad[d] 7: 16$.

$\mathbf{D}(\overline{\mathrm{x}}): \quad[d] 18: 111,[n] 8-4: 51$.

Truncation, estimation: [d]22:256, $[c] 40: 50$.

Bivariate: MR9:363,452.

Flexes equidistant from mode, etc.: $\quad[d] 6: 1$.

Bivariate generalization: $[v] 3: 273$.

Orthogonal Polynomials: Ann. Soc. Cien. Argentina $155: 3$.

Generalizations: $[d] 5: 124,[c] 26: 129,($ cf No. 8.52), MR10:386, MR17:1095, [d]21:289, J. Gakugei Tokushima Univ. Math. 5:29, [n]12-2:95.

Log Pearson distributions: Intl. Cong. Math. (1950) $1: 580$.

Romanovsky's generalization: $[c] 1 \%: 106,[c] 18$ : 221.

See also: $[d] 8: 18,[d] 8: 206,[d] 20: 461,[e] 6: 415$, $[c]^{7}: 127,[c] 16: 106,[c] 16: 198,[c] 18: 264,[c] 20: 389$, $[g] 26(P): 288,[a] 85: 488,[c] 35: 113,[c] 32: 81,[c] 36: 151$, [c]38:4, [i]25:141, MR17:169,272, Z9:314, Z6:268, 719:73, MR14:755,977, Intl. Cong. Math. (1950) $1: 585$.

\subsection{Bessel Function}

Mahalanobis' Distribution ("D" distribution): [e] 2:143,385, $[e] 3: 105,[e] 4: 19,373,535,[e] 8: 167,[k] 8:$ 379, [14]246, MR4:23.

Wilk's distribution of dispersion determinant, etc.: $[e] 3: 26$.

$\mathrm{D}(\overline{\mathrm{x}}): \quad$ MR5:42.

Distribution of vector correlation: $\quad[c] 28: 353$.

Distribution of difference of $\chi^{2}$ variables: $\quad[d] 7: 51$.
$\mathrm{D}(\mathrm{x}), \mathrm{Ch}(\mathrm{x})$, Moments in a special case: $\quad$ MR14: 775 .

D(ratio Type III variables): Studies Presented to Richard von Mises. Academic Press, 1954. p. 301 .

Bivariate Gamma distribution: $\quad[e] 5: 140$.

Distribution of the range: $[d] 18: 384,[d] 21: 133$.

Marginal total of Elfving's distribution: $\quad[c] 36: 142$.

See also: $\quad[e] 6: 175,[e] 8: 235,[a] 97: 125,[a] 98: 89$, $[b] 16: 96,[c] 21: 168,[c] 24: 441,[c] 24: 485,492,[d] 18: 392$, [c]24:39, [c]24:293, [17]Nos. 10,60,61,62,63,64, with Ch $(x)$, refs. $[c] 21: 164,[c] 24: 293,[c] 24: 39$, Several forms, J. Soc. Stat. Paris 96:262, [u]28:458, MR11: 607, MR16:152.

\subsection{Variance Ratio}

$\mathrm{D}(\mathrm{x})=\frac{2\left(1-\rho^{2}\right)^{\frac{1}{2}(\mathrm{n}-1)} \mathrm{x}^{\mathrm{n}-2}\left(1-4 \rho^{2} \mathrm{x}^{2}\left(1+\mathrm{x}^{2}\right)^{-2}\right)^{-\frac{1}{2} \mathrm{n}}}{\mathrm{B}\left[\frac{1}{2}(\mathrm{n}-1), \frac{1}{2}(\mathrm{n}-1)\right]\left(1+\mathrm{x}^{2}\right)^{\mathrm{n}-1}}$,

"Bose", [2]365, [e]2:65.

For $\rho=0, \mathrm{D}\left[(2 \mathrm{n}-3) \mathrm{x}^{2}\right]=$ Hotelling $(2 \mathrm{n}-2, \mathrm{n}-1)$.

See also: $\quad[p] 6: 183,[p]^{\gamma}: 98,[c] 30: 190,[c] 31: 9$.

\subsection{Kullbach}

$\mathrm{D}(\mathrm{x})=\frac{\mathrm{nx} \mathrm{x}^{\mathrm{np}-1}}{\Gamma(\mathrm{n})[\Gamma(\mathrm{p})]^{2}} \sum_{\mathrm{j}=0}^{\infty}(-1)^{\mathrm{n}+\mathrm{nj}+1}\left(\frac{\mathrm{d}^{\mathrm{n}-1}}{\mathrm{dt}^{\mathrm{n}-1}} \frac{\mathrm{x}^{\mathrm{nt}}}{\Gamma(\mathrm{t}+1)}\right)_{\mathrm{t}=\mathrm{j}}$

Distribution of GM from Type III $(1, \mathrm{p})$ : [2]251.

8.5. Noncentral Student

$\mathrm{D}(\mathrm{x}): \quad[c] 31: 362,[18] 1-162,[v] 4: 173,30 \%$.

Multivariate: $[v] 4: 331$.

Application: $\quad[c] 43: 219$.

See also: $\quad[i] 36($ Suppl.):21, $[r] 1: 28$, J. Soc. Stat. Paris 96:262, MR15:46.

\subsection{Continuous $L-x i a n$}

$D(x)=\int_{0}^{1} f(p)\left(\begin{array}{l}n \\ x\end{array}\right) p^{x}(1-p)^{n-x} d p$; parameters; if $f(p)$ is Beta, $\mathrm{D}(\mathrm{x})$ is hypergeometric: $\quad[i] 31: 1,[i] 34: 197$.

8.7. Noncentral Szedecor

$\mathrm{D}(\mathrm{x}): \quad[c] 36: 220,[18] 1-163,[\mathrm{e}] 15: 321$.

See also: $\quad[c] 38: 112,[i] 36($ Suppl.):33, $[r] 3: 33$.

\subsection{Fisher's Logarithmic Series}

$\mathrm{D}(\mathrm{x})=\frac{\mathrm{k}^{\mathrm{x}}}{\mathrm{p} \log \frac{1}{1-\mathrm{k}}}, \mathrm{x}=1,2, \ldots: \quad[\mathrm{c}] 35: 6,[\mathrm{f}] 5: 162$.

Cf. negative binomial $(1,-\mathrm{m})$, No. 3.5: $\quad[c] 37$ :358. 
8.9. Rank Variate

$\mathrm{D}(\mathrm{x})=\frac{\mathrm{N}}{\sigma} \frac{\mathrm{n} !}{(\mathrm{q}-1) !(\mathrm{n}-\mathrm{q}) !}\left[\exp -\frac{\mathrm{x}}{\sigma}(\mathrm{n}-\mathrm{q}+1)\right]$ $X\left(1-\mathrm{e}^{-\mathrm{x} / \sigma}\right)^{\mathrm{q}-1}: \quad[c] 24: 231,239,[c] 25: 79$.

In special case called Yule's distribution, MLE: $[c] 42: 23,425,[c] 43: 248$.

A distribution of Type III median: $\quad[p]^{\%}: 153$.

\subsection{Generalized Pareto}

$\mathrm{D}(\mathrm{x})=\mathrm{ax}^{-\mathrm{n}}\left[1 /\left(\mathrm{e}^{\mathrm{b} / \mathrm{x}}-1\right)\right]: \quad[l] 6: 184$.

$\mathrm{b}=1, \mathrm{n}=5, \mathrm{a}=15 / \pi^{4}$, Planck's radiation function: $[17]$ No. 56 .

\subsection{Ghosh}

$\mathrm{D}(\mathrm{x})=\frac{2}{\Gamma([\mathrm{k}]+1)} \mathrm{x}^{[\mathrm{k}]} \mathrm{e}^{-\mathrm{x}^{2}}$, where $[\mathrm{k}]$ is the largest integer $\leq \mathrm{k}$.

Furnishes counterexample to theorem on similar regions: $[e] 8: 330$.

\subsection{Rectangular Geometric Mean}

$\mathrm{D}(\mathrm{x})=\frac{\mathrm{n}^{\mathrm{n}} \mathrm{x}^{\mathrm{n}-1}}{\mathrm{a}^{\mathrm{n}} \Gamma(\mathrm{n})}(\log \mathrm{a} / \mathrm{x})^{\mathrm{n}-1}: \quad[2] 246,[d] 5: 276,[w] 1: 73$. 8.13. Cauchy Median

$\mathrm{D}(\mathrm{x})=\frac{(2 \mathrm{~m}+1) !}{(\mathrm{m} !)^{2} \pi^{2 \mathrm{~m}+1}}\left(\frac{\pi^{2}}{4}-\left[\mathrm{tan}^{-1}(\mathrm{x}-\mathrm{k})\right]^{2}\right)^{\mathrm{m}} \frac{1}{1+(\mathrm{x}-\mathrm{k})^{2}}:$ $[3] 46$.

\subsection{Spearman's Rank Correlation}

$\mathrm{D}(\mathrm{x}): \quad[c] 30: 256,[c] 34: 183,[c] 38: 131$.

$\sim \mathrm{D}(\mathrm{x})=$ Type II, moments : $\quad[c] 40: 409$.

\subsection{Circular Normal Correlation}

$\mathrm{D}(\mathrm{x})=\mathrm{n}(\mathrm{n}-1) \mathrm{e}^{-\mathrm{x}^{2} / \mathrm{v}}\left(1-\mathrm{e}^{-\mathrm{x}^{2} / 2 \mathrm{v}}\right)^{\mathrm{n}-2}(\mathrm{x} / \sigma): \quad[c] 39: 139$, $[\mathrm{g}] 48: 496$.

\subsection{Koopman}

$\mathrm{D}(\mathrm{x})=\mathrm{Q}(\mathrm{k}) \mathrm{R}(\mathrm{x})$ exp $\mathrm{k} \mathrm{H}(\mathrm{x})$, most general distribution admitting a sufficient estimate of $\mathrm{k}$ : [3]24, [d]23:403, [c]36:71, Trans Am. Math. Soc. 39:399, $[p] 7: 162$.

\subsection{Von Mises}

$\mathrm{D}(\mathrm{x})=\mathrm{C} \exp \mathrm{k} \cos (\mathrm{x}-\mathrm{a}): \quad$ Physikal. Zeitschr 19:490.

'Circular Normal': $[g] 48: 131,[g] 49: 53,268,[c] 43:$ $344,[d] 26: 233,[c] 43: 344$.

\subsection{8.}

Family of distributions having all moments equal: $[d] 11: 402$.

$$
\mathrm{D}(\mathrm{x})=\frac{1}{6} \mathrm{e}^{-\mathrm{x}^{\frac{1}{2}}}\left(1-\mathrm{p} \sin \mathrm{x}^{\frac{1}{1}}\right) \quad 0 \leq \mathrm{x}<\infty, 0 \leq \mathrm{p} \leq 1 .
$$$$
\alpha_{\mathbf{k}}=\frac{1}{6}(4 \mathrm{k}+3) \text { !. }
$$

8.19.

Family of distributions having all moments equal $\mathrm{D}(\mathrm{x})=\mathrm{e}^{-\frac{1}{4}} \pi^{-\frac{1}{2}} \mathrm{x}^{-\log \mathrm{x}}[1-\mathrm{p} \sin (2 \pi \log \mathrm{x})]$, $\alpha_{\mathrm{k}}=\exp \left[\frac{1}{4} \mathrm{k}(\mathrm{k}+2)\right]: \quad[d] 11: 402$.

8.20 .

"Non-null t $\mathrm{t}^{2}$ distribution", involving a hypergeometric function: $[14] 48$.

\subsection{1 .}

$\mathrm{D}(\mathrm{x})=\frac{\operatorname{sech}^{\mathrm{k}-2} \mathrm{x}}{\mathrm{B}(1, \mathrm{k}-2)}$, the distribution of $\tanh ^{-1} \mathrm{r}$ in samples from a bivariate normal distribution with zero means and zero correlation: [b]15:213, $[b] 9: 61$.

$\mathrm{Ch}(\mathrm{x})$, also special cases and refs: $\quad[17]$ Nos. 53-5. $\mathrm{D}\left(\Sigma \mathrm{x}_{\mathrm{i}}\right)$ for $\mathrm{k}=3$ : $\quad$ Z9:219.

\subsection{2 .}

$\mathrm{D}(\mathrm{x})=\frac{1}{2 \mathrm{a}} \operatorname{sech}^{2}\left(\frac{\mathrm{x}-\mathrm{m}}{\mathrm{a}}\right)$, connected with lognormal: [e]12:122.

Called 'logistic', $\mathrm{Ch}(\mathrm{x})=\pi \mathrm{x} \operatorname{sech} \pi \mathrm{x}$ when $\mathrm{m}=0, \mathrm{a}=2$ : [v] $7: 163$.

Called 'hyperbolic law of errors', properties, examples: $[s] 2: 55$.

Distribution of $\mathrm{t}$ and $\mathrm{F}$ statistics: $M R$ 13:665.

$$
8.23 \text {. }
$$

Distribution of the correlation ratio, involving series: $\quad[a] 97: 121,[c] 24: 441$.

\subsection{4 .}

Various distributions of the form exp (-quartic polynomial): $\quad[d] 4: 1,[d] 4: 79,[d] 19: 589$.

Giving an example where no minimum variance estimator exists: $\quad[e] 12: 43$.

MLE: $\quad[c] 31: 188,[a] 98: 114$.

$$
8.25 \text {. }
$$

$\mathrm{k}\left(1+\mathrm{x}^{2}\right)^{-\mathrm{m}}: \quad$ [2]52.

$\mathrm{Ch}(\mathrm{x}): \quad[2] 6 \%$.

8.26 .

$\mathrm{D}(\mathrm{x})=\mathrm{b} \sin 2(\mathrm{a}+\mathrm{bx}): \quad[b] 9: 61$.

8.27 .

Normal multiplied by an eighth degree polynomial: $[a] 106: 361$.

8.28 .

$\mathrm{D}(\mathrm{x})=\left(\frac{1}{4} \mathrm{~h}+\frac{1}{4} \mathrm{~h}^{2}|\mathrm{x}|\right) \mathrm{e}^{-\mathrm{h}|\mathrm{x}|}, \mathrm{D}(\overline{\mathrm{x}}): \quad[n] 10-3: 90$.

For $\mathrm{h}=1, \mathrm{Ch}(\mathrm{x})=\left(\frac{1}{1+\mathrm{x}^{2}}\right)^{2}: \quad[n] 10: 75,[17]$ No.39. 
8.29 .

Miscellaneous distributions given in terms of $\mathrm{C}(\mathrm{x})$ : [d]13:217, Math. Tables and Other Aids to Computation 5:109, [g]50:209.

8.30.

$\mathrm{D}(\mathrm{x})=\frac{1}{2} \mathrm{k}(1+|\mathrm{x}|)^{-\mathrm{k}-1}: \quad[1] 225 \quad$ No. 2.

k negative: $\quad[c] 33: 126$.

$\mathrm{k}=1+\mathrm{p}: \quad[c] 36: 93,[17]$ No. 17, generalization No. $^{7}$ 18.

8.31 .

$\mathrm{D}(\mathrm{x})=\mathrm{k} \exp \left(-a x^{\mathrm{p}}\right)\left[1+\mathrm{q} \sin \left(\mathrm{bx}^{\mathrm{p}}\right)\right]: \quad$ [2]106.

8.32 .

Various distributions formed from rational functions of $\mathrm{x}$, rational functions multiplied by $\mathrm{e}^{-1 / \mathrm{x}}, \mathrm{e}^{-\mathrm{x}}$ and $\exp \left(-\tan ^{-1} x\right): \quad[d] 1: 137$.

8.33.

$\mathrm{D}(\mathrm{x})=\left(\mathrm{e}^{2}+|\mathrm{x}|\right)^{-1}\left[\log \left(\mathrm{e}^{2}+|\mathrm{x}|\right)\right]^{-2}$, having a pathologically long tail: $[d] 17: 11$.

\subsection{Weibull}

$\mathrm{D}(\mathrm{x})=\mathrm{ab} \quad \mathrm{x}^{\mathrm{b}-1} \quad \exp (-\mathrm{ax}), \quad \mathrm{x} \geq 0, \quad \mathrm{a}>0, \quad \mathrm{~b}>1$ : $[g] 43: 408,[17]$ No. 44

Moments of order statistics: $\quad[d] 26: 330$.

8.35.

$\mathrm{D}(\mathrm{x})=\mathrm{k} \sin ^{\mathrm{m}} \mathrm{x} \cos ^{\mathrm{n}} \mathrm{x}: \quad[c] 30: 182$.

$\mathrm{n}=0$, value of $\mathrm{k}, \mathrm{Ch}(\cos \mathrm{x})$ : Phil. Mag. Ser. 7 , $39: 70$

8.36 .

$\mathrm{D}(\mathrm{x})=2 \mathrm{~h} \pi^{-1}\left(1+\mathrm{h}^{2} \mathrm{x}^{2}\right)^{-2}: \quad[n] 10-3: 7 \%$

8.37.

$\mathrm{D}(\mathrm{x})=2 \mathrm{~h} \pi^{-1}\left(\mathrm{e}^{\mathrm{hx}}+\mathrm{e}^{-\mathrm{hx}}\right)^{-1}, \quad \mathrm{D}(\overline{\mathrm{x}}) \quad$ called "Perks": $[n] 10-3: 90,[d] 26: 153,[v] \%: 159, J$. Inst. Actuar. 63:12.

\subsection{8.}

$\mathrm{D}(\mathrm{x})=(2 \pi)^{-\frac{1}{2}}(\mathrm{x} / \mathrm{k})\left[\exp \left(-\frac{1}{2}(\mathrm{x}-\mathrm{k})^{2}\right)\right.$

$\left.-\exp \left(-\frac{1}{2}(\mathrm{x}+\mathrm{k})^{2}\right)\right]$

[2]38\%.

8.39.

Four distributions formed by multiplying the normal distribution by a polynomial, used to illustrate kurtosis: $[\mathrm{g}] 40: 259$.

\subsection{Extreme Value}

$\mathrm{D}(\mathrm{x})=\mathrm{a} \exp [-\mathrm{a}(\mathrm{x}-\mathrm{m})] \exp [-\exp -\mathrm{a}(\mathrm{x}-\mathrm{m})]:$ $[d] 17: 299$.

Gumbel or Fisher-Tippett distribution.
Determination of constants: C.R. Acad. Sci. Paris 222:34.

Estimation, MLE: $\quad[d] 24: 282$.

Bias: $[d] 27: 758$.

$\mathrm{Ch}(\mathrm{x})=\Gamma\left(1-\mathrm{ixa}^{-1}\right) \exp (\mathrm{ixm})$ Cumulants (see reference): $\quad[18] 1-144$.

For $\mathrm{m}=0, \quad \mathrm{a}=1, \quad \mathrm{Ch}(\mathrm{x})=\Gamma(1-\mathrm{ix}), \quad$ References : $[17]$ No. 43, $[u] 24: 180$.

Connection with No. 2.3: $[v] 4: 8, \quad[g] 50: 518$, $[g] 42: 408$.

Special cases: $\quad[r] 1: 4$.

$\sim \mathrm{D}(\mathrm{x}): \quad[u \mid 24: 180,[\mathrm{~g}] 43: 403$, Studies presented to Richard von Mises. Academic Press, 1954 p. 346.

With slight modification $\sim \mathrm{D}(\mathrm{x})$ is distribution of $\log$ survival time: J.Hygiene 42:328.

See also: U.S. Dept. Agric. ARS 41:13, Ann. Inst. Henri Poincaré 4:115, 5:115, J. de Physique Serie 7 Vol. 8, nos. 8, 11, Bull. Am. Meteor. Soc, 23:95, C.R. Acad. Sci. Paris 246:49, 237: 512, Nature 175:270, Cong. Intl. Math. 1936, 2:200, [a]99:732, [w]8:97 NBS Appl. Math. Ser. No. 33.

8.41 .

$\mathrm{D}(\mathrm{x})=\frac{2}{\pi} \frac{\mathrm{x}^{2}}{\left(1+\mathrm{x}^{2}\right)^{2}}, \quad \mathrm{Ch}(\mathrm{x})=\mathrm{e}^{-|\mathrm{x}|}(1-|\mathrm{x}|), \quad[n] 10: 75$, $[17]$ No.30.

8.42 .

$\mathrm{D}(\mathrm{x}+\mathrm{y})$, where $\mathrm{x}$ and $\mathrm{y}$ obey various trivial distributions: $\quad[d] 5: 16$.

8.43 .

$\mathrm{D}(\mathrm{x})=\mathrm{Cx}^{-1}(1+\mathrm{p} / \mathrm{x})^{-2}: \quad[d] 6: 106$.

8.44

$\mathrm{D}(\mathrm{x})=\frac{\mathrm{a}+1}{2 \mathrm{a}}\left(1-|\mathrm{x}|^{\mathrm{a}}\right),-1<\mathrm{x}<1: \quad[17]$ No.15.

8.45 .

$\mathrm{D}(\mathrm{x})=\frac{\lambda^{\mathrm{a}}}{\left(1+\frac{\mathrm{c}}{\lambda^{\mathrm{b}}}\right)^{\mathrm{n}}} \mathrm{e}^{-\lambda \mathrm{x}} \mathrm{x}^{\mathrm{a}-1} \sum_{\mathrm{j}=0}^{\mathrm{n}}\left(\begin{array}{l}\mathrm{n} \\ \mathrm{j}\end{array}\right) \frac{\mathrm{c}^{\mathrm{j}} \mathrm{x}^{\mathrm{bj}}}{\Gamma(\mathrm{bj}+\mathrm{a})}, 0<\mathrm{x}<\infty$, $\lambda>0, c \geq 0, b \geq 0, a>0, n=1,2, \ldots$,

Ch(x), References: $\quad[17]$ No. 36 .

8.46 .

$\mathrm{D}(\mathrm{x})=(\mathrm{x}-\mathrm{k}) \mathrm{x}^{\mathrm{n}} \mathrm{e}^{-\mathrm{ax}}: \quad[g] 42: 572$.

8.47. Stevens-Fisher

$\mathrm{D}(\mathrm{x})=\Sigma\left(\begin{array}{l}\mathrm{n} \\ \mathrm{j}\end{array}\right)(-1)^{\mathrm{j}}(1-\mathrm{jx})^{\mathrm{n}-1} \quad[k] 9: 315,[k] 10: 14$.

Compare No. 5.16 and No. 8.70. 
$\mathrm{D}(\mathrm{x})=\mathrm{C} \exp \left(\mathrm{ax}^{\mathrm{b}}-\mathrm{cx}\right), 0<\mathrm{x}<1: \quad[d] 25: 641$.

8.49 .

$\mathrm{D}(\mathrm{x})=\mathrm{C} \exp \left[-\mathrm{a}(\mathrm{b}-\mathrm{x})^{-\mathrm{c}}\right]: \quad[d] 25: 645$.

8.50 .

$\mathrm{D}(\mathrm{x})=\frac{\mathrm{a}}{2 \Gamma(1 / \mathrm{a})} \mathrm{e}^{-|\mathrm{x}|^{\mathrm{a}}},-\infty<\mathrm{x}<\infty, \mathrm{a}>0$ $[i] 5: 133,[g]$ 26(Suppl.-H)227,[17]No.40.

MLE: $\quad[u] 45: 542$.

8.51 .

Distribution of non-normal correlation: $\quad[c] 38$ :224. 8.52 .

$\mathrm{D}(\mathrm{x})=\mathrm{C}\left[\frac{\mathrm{p}-\mathrm{x}^{2}}{\mathrm{q}+\mathrm{x}^{2}}\right] \frac{\mathrm{m}}{\mathrm{p}+\mathrm{a}},-\sqrt{\mathrm{p}}<\mathrm{x}<\sqrt{\mathrm{p}}$ Value of C, references: $\quad[17]$ No.31.

Hansmann's distributions, obtained from a generalized Pearson differential equation: $\quad[c] 26: 129$.

8.53.

$\mathrm{D}(\mathrm{x})=(\mathrm{k} / \mathrm{x}) \exp [-\mathrm{ax}-(\mathrm{b} / \mathrm{x})]: \quad[17]$ No.48.

Called "Type Harmonique": C.R. Acad Sci. Paris 213:634.

\subsection{4 .}

$\mathrm{D}(\mathrm{x})=(\mathrm{a} / \sqrt{\pi}) \mathrm{e}^{2 \mathrm{a} \sqrt{\mathrm{b}}} \frac{1}{\mathrm{x}^{3 / 2}} \mathrm{e}^{-\mathrm{bx}-\frac{\mathrm{a}^{2}}{\mathrm{x}}}: \quad[\mathrm{i}] 23: 101$.

$\operatorname{Ch}(\mathrm{x})=\exp [2 \mathrm{a}(\sqrt{\mathrm{b}}-\sqrt{\mathrm{b}-\mathrm{it})}]: \quad[17]$ No.50.

8.55.

$\mathrm{D}(\mathrm{x})=\frac{\mathrm{a}^{\mathrm{p}}|\mathrm{r}|}{\Gamma(\mathrm{p})} \mathrm{x}^{\mathrm{rp}-1} \mathrm{e}^{-\mathrm{ax}^{\mathrm{r}}}, 0<\mathrm{x}<\infty, \mathrm{a}>0, \mathrm{p}>0$ :

No.51.

8.56.

$\mathrm{D}(\mathrm{x})=(\mathrm{a}-1)^{2} \quad(\log \mathrm{x}) / \mathrm{x}^{\mathrm{a}}, \quad 1<\mathrm{x}<\infty, \quad 1<\mathrm{a}$ : No.5\%.

\subsection{7.}

$\mathrm{D}(\mathrm{x})=-\left[\mathrm{L}\left(\mathrm{b}^{1-\mathrm{a}}\right) \mathrm{x}^{\mathrm{a}} \log \mathrm{x}\right]^{-1}, \mathrm{~b}<\mathrm{x}<\infty, \mathrm{a}>1, \mathrm{~b}>1$, and

$\mathrm{L}(\mathrm{u})=\int_{0}^{u} \frac{\mathrm{dv}}{\log \mathrm{v}}, \mathrm{u} \geqq 0: \quad[17] N o .58$.

8.58.

$\mathrm{D}(\mathrm{x})=-(\mathrm{a}+1)^{2} \mathrm{x}^{\mathrm{a}} \log \mathrm{x}, \quad 0<\mathrm{x}<1, \quad \mathrm{a}>-1$ : No.59.

8.59.

A generalization of the hypergeometric distribution based on the Whittaker function $\mathrm{x}^{\mathrm{m}+\frac{1}{2}} \mathrm{e}^{-\frac{1}{2} \mathrm{x}}{ }_{1} \mathrm{~F}_{1}\left(\mathrm{~m}+\frac{1}{2}-\mathrm{k}, 2 \mathrm{~m}+\mathrm{x} ; \mathrm{x}\right)$

$\mathrm{D}(\mathrm{x}), \mathrm{Ch}(\mathrm{x})$, references: $[17]$ No.65.
$\mathrm{D}(\mathrm{x})=\mathrm{C} \mathrm{e}^{-\left(\mathrm{x}^{2} / 2 \mathrm{a}^{2}\right)} /\left(\mathrm{b}^{2}+\mathrm{x}^{2}\right)$, moments, Ch( $\left.\mathrm{x}\right)$, limiting cases (Cauchy, Normal): [ [v]2:293, [v]3:139.

8.61.

$\mathrm{D}(\mathrm{x})=(1 / \pi)\left[(1-\cos \mathrm{x}) / \mathrm{x}^{2}\right]: \quad[v] 2: 328$.

\subsection{2.}

$\mathrm{D}(\mathrm{x})$ where $\mathrm{A}+\mathrm{Bf}(\mathrm{x})$ is (a) normal, or (b) Laplace and $f(x)$ is (i) $\log x$, (ii) $\log [x /(1-x)]$, (iii) $\operatorname{arcsinh}$ $\mathrm{x}: \quad[c] 36: 149,[v] 5: 283$.

8.63.

$\mathrm{D}(\mathrm{x})=\left\{\begin{array}{r}1-\mathrm{e}^{-\mu \mathrm{NT}}, \quad \mathrm{x}=\mathrm{N} \\ \left(1-\mathrm{e}^{-\mu \mathrm{T}}\right) \exp [-\mu \mathrm{T}(\mathrm{x}-1)], \quad \mathrm{x}=\mathrm{N}+1, \\ \mathrm{~N}+2, \ldots\end{array}\right.$ $\mathrm{N}=1,2, \ldots, \quad \mu>0, \mathrm{~T}>0$.

\subsection{4.}

Garwood's distribution of length of gaps in traffic: $[b]^{7}: 65,[g] 46: 11 \%,[c] 38: 384$.

\subsection{Matching}

$\mathrm{D}(\mathrm{x})=(1 / \mathrm{x} !)[1-(1 / 1 !)+(1 / 2 !)-\ldots+$ $\left.(-1)^{\mathrm{n}-\mathrm{x}} /(\mathrm{n}-\mathrm{x}) !\right]: \quad[a] 118: 390, \dot{M} \dot{\mathrm{M}} 18: 346$.

Generalization: [4]210.

8.66 .

Cigarette card distribution: $\quad[a] 118: 391$.

\subsection{7.}

Cubic polynomial over a finite range, estimation: [g] $50: 196,[d] 26: 505,591$.

$D(x)=\left\{\begin{array}{ll}\frac{m n}{m+n} x^{m-1}, & 0<x<1 \\ \frac{m n}{m+n} x^{-n-1}, & 1 \leqq x<\infty\end{array}\right\}$

Moments, etc.: $\quad[g] 50: 113 \%$.

\subsection{9.}

$D(x)=\left\{\begin{array}{l}m n(m-n)^{-1} x^{n-1}\left(1-x^{m-n}\right), m \neq n \\ n^{2} x^{n-1} \log (1 / x), \quad m=n\end{array}\right\}$

Moments: [g]50:1142.

8.70.

$D(x)=\frac{n^{n}}{(n-1) !} \sum_{j \leqq n x}(-1)^{j}\left(\begin{array}{c}n \\ j\end{array}\right)\left(x-\frac{j}{n}\right)^{n-1}, 0 \leqq x \leqq 1$

Compare Nos. 5.16 and 8.47: [d]26:713. 
8.71.

$\mathrm{D}(\mathrm{x})=\frac{(\mathrm{r}-1) !(\mathrm{r}-\mathrm{x})}{\mathrm{x} ! \mathrm{r}^{\mathrm{r}-\mathrm{x}}}, \mathrm{x}=0,1, \ldots,(\mathrm{r}-1)$

Moments, approximations: [b]19:339.

\subsection{Arfwedson}

$\mathrm{D}(\mathrm{x})=\sum_{\mathrm{j}=0}^{\infty}(\mathrm{x}-\mathrm{j})^{\mathrm{n}}(-1)^{\mathrm{j}}\left(\begin{array}{l}\mathrm{x} \\ \mathrm{j}\end{array}\right) \quad[i] 34: 121$.

\subsection{Stevens-Craig}

$\mathrm{D}(\mathrm{x})=\mathrm{C} \mathrm{n}^{(\mathrm{x})} \sigma_{\mathrm{s}}^{\mathrm{x}}, \sigma_{\mathrm{s}}^{\mathrm{x}}$ being Stirling's number of second kind: $\quad[k] 8: 57,[c] 40: 173$.

Generalization: $[w]$ : 203 .

\subsection{4 .}

$\mathrm{D}(\mathrm{x})=\frac{1}{4}+\mathrm{x}^{4}, \quad-1<\mathrm{x}<1: \quad[m] 6: 120$.

\subsection{Ising-Stevens}

$\left.\mathrm{D}(\mathrm{x})=\frac{\left(\begin{array}{c}\mathrm{m}-1 \\ \mathrm{x}-1\end{array}\right)\left(\begin{array}{c}\mathrm{m}+1 \\ \mathrm{x}\end{array}\right)}{\left(\begin{array}{c}\mathrm{m}+\mathrm{n} \\ \mathrm{m}\end{array}\right)}\right\}\left\{\begin{array}{l}\text { Zeit. f. Physik 31:253, } \\ {[k] 9: 10,[d] 11: 370,} \\ {[t] 4: 171,[w] 8: 55 .}\end{array}\right.$

8.76.

$\mathrm{D}(\mathrm{x})=\frac{1}{\pi \sqrt{\mathrm{m}^{2}-\mathrm{x}^{2}}}: \quad$ Ann. of Math. $27: 18$.

8.77.

$\mathrm{D}(\mathrm{x})=\frac{\mathrm{x}^{-\frac{2}{3}}}{3 \sqrt{2 \pi}} \exp \left[-\frac{1}{2}\left(\mathrm{x}^{\frac{1}{3}}-\mathrm{b}\right)^{2}\right] \quad$ Ann. of Math. 19. 27 :

\subsection{Negative Hypergeometric}

$\mathrm{D}(\mathrm{x})=\left(\begin{array}{l}\mathrm{n} \\ \mathrm{x}\end{array}\right) \frac{\mathrm{B}(\mathrm{p}+\mathrm{x}, \mathrm{q}+\mathrm{n}-\mathrm{x})}{\mathrm{B}(\mathrm{p}, \mathrm{q})}: \quad[b] 10: 25 \%$, Proc.

Int'l. Stat. Conf. Rome 1953 paper 71.

Obtained by assuming binomial probability to obey Beta.

\subsection{9.}

$\mathrm{D}(\mathrm{x})=\mathrm{k}(1+\mathrm{x})^{-2}$ over various ranges: $\quad[d] 22: 425$.

8.80 .

$\mathrm{D}(\mathrm{x})=(\mathrm{n}-1)\left[1-(1 / \mathrm{x})^{\mathrm{n}-2}\right]\left(1 / \mathrm{x}^{2}\right), \quad \mathrm{x} \geq 1: \quad[d] 22: 425$.

8.81 .

$\mathrm{D}(\mathrm{x})=-\log \mathrm{x}^{2} / \pi^{2}\left(1-\mathrm{x}^{2}\right), \quad-\infty<\mathrm{x}<\infty: \quad[d] 22: 425$.

8.82.

$\mathrm{D}(\mathrm{x})=\frac{\mathrm{a}^{-(\mathrm{a}-\mathrm{bx})^{2} / 2 \mathrm{cx}}}{\sqrt{2 \pi \mathrm{cx}^{3}}}$ Called "inverse Gaussian":

Nature 155:453, [u]43:41, Virginia J. Sci. (new series) $7: 160$.
Written

$\mathrm{D}(\mathrm{x})=\frac{\exp \left(\frac{-\mathrm{c}(\mathrm{x}-\mathrm{m})^{2}}{2 \mathrm{~m}^{2} \mathrm{x}}\right)}{\left(\frac{\mathrm{c}}{2 \mathrm{x}^{3}}\right)^{\frac{1}{2}}}: \quad$ [d]28:362, 696.

$\mathrm{D}(\mathrm{x})=\mathrm{k} \quad \mathrm{x}^{\mathrm{n}} \mathrm{e}^{-\mathrm{x}^{2}+\mathrm{ax}}$, called "Halphen,": Publ. Inst. Statist. Univ. Paris 4:38.

8.83 .

$\mathrm{D}(\mathrm{x})=\mathrm{a}(\alpha, \beta) /\left[\exp \left(\alpha^{2} \mathrm{x}^{2}\right)-\beta\right]: \quad$ MR16:381.

8.84 .

$\mathrm{D}(\mathrm{x})=\mathrm{a} \exp \left[-\mathrm{k}^{2} \log ^{2} \rho\right]$ where

$\rho=\left[\left(\mathrm{x}-\mathrm{x}_{0}\right)\left(\mathrm{x}_{2}-\mathrm{x}_{1}\right)\right] /\left[\left(\mathrm{x}_{2}-\mathrm{x}\right)\left(\mathrm{x}_{1}-\mathrm{x}_{0}\right)\right]: \quad 210: 313$.

8.85 .

$\mathrm{Ch}(\mathrm{x})=1 / \cosh \mathrm{t}, \mathrm{t} / \sinh \mathrm{t}, 1 / \cosh ^{2} \mathrm{t}: \quad$ MR11:443.

8.86 .

$\mathrm{D}(\mathrm{x})=2 \lambda(\lambda+1) /(\mathrm{x}+\lambda-1)(\mathrm{x}+\lambda)(\mathrm{x}+\lambda+1), \lambda>0$, $\mathrm{x}=1,2, \ldots, \mathrm{m}=1+\lambda, \mathrm{v}=\infty$ : [e]18:353.

$\mathrm{D}(\mathrm{x})=\frac{\mathrm{N} ! \mathrm{n} !}{\prod_{\mathrm{i}=0}^{\mathrm{n}} \mathrm{x}_{1} !(\mathrm{i} !)^{\mathrm{x}_{\mathrm{i}}}}: \quad[s] 5: 161$.

8.88.

$\mathrm{D}(\mathrm{x})=\mathrm{ce}^{\mathrm{cx}} /\left(1+\mathrm{e}^{\mathrm{cx}}\right)^{2}: \quad[s] 1: 55,[s] 3: 133$.

Hyperbolic Error distribution.

A discrete distribution from an urn model:

$[d] 22: 452,[w] 7: 173$.

\subsection{9.}

$\mathrm{D}(\mathrm{x})=\frac{\mathrm{e}^{-\lambda \mathrm{x}}}{\alpha \mathrm{x}} \sum_{\mathrm{n}=1}^{\infty} \frac{1}{[\Gamma(\mathrm{n})]^{2}}\left(\lambda \mathrm{x} \alpha \mathrm{e}^{-\alpha}\right)^{\mathrm{n}} \mathrm{n}^{\mathrm{n}-2}, 0<\mathrm{x}<\infty$, $0<\alpha<1, \lambda>0, \mathrm{~m}=\frac{1}{\lambda(1-\alpha,}, \mathrm{v}=\frac{1-\alpha+\alpha^{2}}{\lambda^{2}\left(1-\alpha^{3}{ }^{3}\right.}$.

8.90 .

$\mathrm{D}(\mathrm{x})=\mathrm{k}^{\mathrm{x}} \sum_{\mathrm{j}=0}^{\mathrm{x}-1} \frac{\left(\begin{array}{c}\mathrm{x}-\mathrm{1} \\ \mathrm{j}\end{array}\right) \mathrm{p}^{\mathrm{j}}}{(\mathrm{x}-\mathrm{j}) !}, \quad \mathrm{x}=0,1, \ldots$

Called "Pólya-Aeppli": $\quad[c] 40: 206$.

8.91.

$\mathrm{D}(\mathrm{x})=\frac{1}{\mathrm{x}}\left(\begin{array}{c}2 \mathrm{x}-2 \\ \mathrm{x}-1\end{array}\right) \mathrm{k}^{\mathrm{x}-1} /(1+\mathrm{k})^{2 \mathrm{x}-1}, \quad \mathrm{x}=1,2, \ldots$

8.92.

$\mathrm{D}(\mathrm{x})= \begin{cases}\left(\frac{\lambda}{\lambda+\mu}\right)^{\mathrm{N}}\left(\mu^{\mu \mathrm{x}}\right) \Gamma(\mathrm{N},(\lambda+\mu) \mathrm{x}) / \Gamma(\mathrm{N}), & \mathrm{x}>0 \\ \left(\frac{\lambda}{\lambda+\mu}\right)^{\mathrm{N}}\left(\mu \mathrm{e}^{\mu \mathrm{x}}\right), & \mathrm{x}<0\end{cases}$ 
$\mathrm{D}(\mathrm{y}-\mathrm{z})$ where $\mathrm{D}(\mathrm{y})=$ Type III $(\lambda, \mathrm{N}), \mathrm{D}(\mathrm{z})=$ Type III $(\mu, 1)$

$$
\begin{aligned}
& \alpha_{\mathrm{k}}=(-\mu)^{-\mathrm{k}} \mathrm{k} ! \sum_{\mathrm{i}=0}^{\mathrm{k}}\left(\begin{array}{c}
\mathrm{i}+\mathrm{N}-1 \\
\mathrm{i}
\end{array}\right)(-\mu / \lambda)^{2} \\
& \kappa_{\mathrm{k}}=(\mathrm{k}-1) !\left[\frac{\mathrm{N}}{\mu^{\mathrm{k}}}+(-1)^{\mathrm{k}} / \lambda\right]
\end{aligned}
$$

\subsection{Miller}

$\mathrm{D}(\mathrm{x})=(\mathrm{k}+1) \frac{(\mathrm{m}+\mathrm{k}+1) !}{\mathrm{m} !} \frac{(\mathrm{m}+\mathrm{x}-1) !}{(\mathrm{m}+\mathrm{k}+\mathrm{x}+1) !}, \quad \mathrm{x}=0,1, \ldots$ $\alpha_{1}=\frac{\mathrm{m}+\mathrm{k}+1}{\mathrm{k}}, \quad \sigma^{2}=\frac{(\mathrm{m}+\mathrm{k}+1)(\mathrm{k}+1)(\mathrm{m}+1)}{\mathrm{k}^{2}(\mathrm{k}-1)}$.

Obtained by assuming Pascal parameter to be Beta.

\section{Miscellaneous Bivariate}

9.1. Cauchy Bivariate
$\mathrm{D}(\mathrm{x}, \mathrm{y})=(1 / \pi)\left[1 /\left(1+\mathrm{x}^{2}+\mathrm{y}^{2}\right)\right]^{2}: \quad[5] .45$
$\mathrm{D}(\mathrm{x}, \mathrm{y})=\frac{1}{2 \pi}\left(1+\mathrm{x}^{2}+\mathrm{y}^{2}\right)^{-3 / 2}: \quad[w] 8: 235$.

\subsection{Student Bivariate}

$\mathrm{D}(\mathrm{x}, \mathrm{y})=(2 \pi)^{-1}\left(1-\mathrm{r}^{2}\right)^{-\frac{1}{2}}\left(1+\frac{\mathrm{x}^{2}-2 \mathrm{rxy}+\mathrm{y}^{2}}{\mathrm{n}\left(1-\mathrm{r}^{2}\right)}\right)^{-\frac{1}{2}(\mathrm{n}+2)}$

Tables: $\quad[c] 22: 408,[c] 41: 154$.

If $x, y$ independent: [3]92.

\subsection{Poisson Bivariate}

Discussion: [2]136, [f]\%:414, [c]39:196, Psych. Bull. 47:434, Proc. Edin. Math. Soc. IIs 4:18.

Special case obtained from binomial: $\quad[i] 17: 98$.

$$
9.4 \text {. }
$$

Lognormal bivariate: $\quad[c] 22: 130,[d] 4: 30$.

\section{5 .}

Normal-lognormal: $[d] 4: 30$.

\subsection{Binomial Bivariate}

$\operatorname{Ch}(x, y)=\left(a \exp (\text { is }+ \text { it })+b e^{i s}+c e^{i t}+d\right)^{n}:$

[2]133.

If $a=0, D(x, y)=\frac{k !}{x ! y !(k-x-y) !} p^{x} q^{y}(1-p-q)^{k-x-y}$, etc.: $\quad[i] 17: 92,[i] 19: 209$.

See also: [i] $] 36: 74$, MR14:995, Z18:154, MR13: 665 .

\subsection{Gamma Bivariate}

$\operatorname{MGF}(\mathrm{x}, \mathrm{y})=\left[(1+\mathrm{s})(1-\mathrm{t})-\mathrm{str}^{2}\right]^{-\mathrm{p}}$ : [c]25:158, MR3:171.
9.8. Gamma-Normal

$\operatorname{MGF}(\mathrm{x}, \mathrm{y})=(1-\mathrm{s})^{-\frac{1}{2}} \exp \left[\frac{1}{2} \mathrm{t}^{2}\left(1+\frac{\mathrm{sr}^{2}}{1-\mathrm{s}}\right)\right]: \quad[e] 5: 144$. $[c] 25: 132$.

\subsection{Hypergeometric Bivariate}

$\mathrm{D}(\mathrm{x}, \mathrm{y})=\frac{\left(\begin{array}{l}\mathrm{a} \\ \mathrm{x}\end{array}\right)\left(\begin{array}{l}\mathrm{b} \\ \mathrm{y}\end{array}\right)\left(\begin{array}{c}\mathrm{c} \\ \mathrm{k}-\mathrm{x}-\mathrm{y}\end{array}\right)}{\left(\begin{array}{c}\mathrm{a}+\mathrm{b}+\mathrm{c} \\ \mathrm{k}\end{array}\right)}$, various properties:

$[i] 17: 104,[c] 16: 172,[c] 22: 140$.

Moments: Ganiia 5:97, Koninkl. Nederl. Akad (A) $60: 124$.

\subsection{Negative Binomial Bivariate}

Various properties: $[i] 1 \%: 100$.

$\mathrm{D}(\mathrm{x}, \mathrm{y})=\frac{\mathrm{p}^{\mathrm{p}}}{(\mathrm{p}+2 \mathrm{~m}) \mathrm{p}} \frac{1}{\Gamma(\mathrm{p})} \frac{\Gamma(\mathrm{x}+\mathrm{y}+\mathrm{p})}{\Gamma(\mathrm{x}+1) \Gamma(\mathrm{y}+1)}\left[\frac{\mathrm{m}}{\mathrm{p}+2 \mathrm{~m}}\right]^{\mathrm{x}+\mathrm{y}}$ correlation $=\frac{\mathrm{m}}{\mathrm{p}+\mathrm{m}}$, regression etc. $: \quad[c] 41: 79$.

Polya-Eggenberger: MR11:605.

\subsection{Elfving}

$\mathrm{D}(\mathrm{x}, \mathrm{y})=\frac{1}{2} \mathrm{x} \exp (-\mathrm{x} \cosh \mathrm{y})$, connected with $\sim \mathrm{D}$ (range): $[c] 34: 111,[c] 36: 142$.

9.12 .

$\mathrm{D}(\mathrm{x}, \mathrm{y})=\mathrm{C} \mathrm{e}^{-\mathrm{ax}-\mathrm{by}}(1-\mathrm{x}+\mathrm{y})^{\mathrm{p}}(1+\mathrm{x}-\mathrm{y})^{\mathrm{q}}: \quad[c] 14:$ 355.

Rhodes surface: $[c] 22: 134,[c] 41: 550$.

\subsection{3.}

$\mathrm{D}(\mathrm{x}, \mathrm{y})=(1+\mathrm{x} / \mathrm{a})^{\mathrm{m}}(1+\mathrm{y} / \mathrm{b})^{\mathrm{n}}[1-(\mathrm{x}+\mathrm{y}) / \mathrm{c}]^{\mathrm{a}}$, FilonIsserlis surface: $\quad[c] 15: 222,[c] 16: 180$.

9.14 .

$\mathrm{D}(\mathrm{x}, \mathrm{y})=(\mathrm{xy})^{\mathrm{k}}(\mathrm{x}-\mathrm{y})[(1-\mathrm{x})(1-\mathrm{y})]^{\mathrm{n}}: \quad(c) 31: 226$, $[k]: 245$.

9.15.

$\mathrm{D}(\mathrm{x}, \mathrm{y})=\frac{\mathrm{x}^{\mathrm{n}-2}\left(1-\mathrm{y}^{2}\right)^{\frac{1}{2}(\mathrm{n}-4)}}{\left(1-2 \mathrm{rxy}+\mathrm{y}^{2}\right)^{\mathrm{n}-1}}: \quad$ [2]365.

9.16 .

$\mathrm{D}(\mathrm{x}, \mathrm{y})=\mathrm{n}^{-2} \frac{\mathrm{n} !}{[(\mathrm{k}-1) !]^{2}(\mathrm{n}-2 \mathrm{k}) !}(\mathrm{x} / \mathrm{n})^{\mathrm{k}-1}(\mathrm{y} / \mathrm{n})^{\mathrm{k}-1}(1-$ $\mathrm{x} / \mathrm{n}-\mathrm{y} / \mathrm{n})^{\mathrm{n}-2 \mathrm{k}}$ $\mathrm{x}>0, \mathrm{y}>0, \mathrm{x}+\mathrm{y}<\mathrm{n}, 2 \mathrm{k}<\mathrm{n}$

As $n \rightarrow \infty, x, y \rightarrow$ independent Type $\operatorname{III}(1, k)$

Special case, $\mathrm{k}=0: \quad[d]^{7}: 149$. 
$\mathrm{D}(\mathrm{x}, \mathrm{y})=\mathrm{C} \quad \mathrm{x}^{\frac{1}{2}(\mathrm{n}-3)} \quad(\mathrm{y}-\mathrm{x})^{\frac{1}{2}(\mathrm{k}-\mathrm{n}-2)} \quad \exp \quad\left[-\frac{1}{2}(\mathrm{k}-1) \mathrm{y}\right]$, $\mathrm{D}(\mathrm{x} / \mathrm{y})=$ Beta : $\quad[b] 1: 213$.

9.18.

Uniform bivariate, triangular bivariate: [c]24: 382, $[v] 5: 322$.

\subsection{9.}

Gram-Charlier bivariate: $\quad[c] 36: 17 \%$.

9.20 .

The fifteen constant surface,

(quartic polynomial) $\cdot \mathrm{e}^{-\mathrm{Q}:} \quad[c] 17: 268$.

\subsection{1 .}

Pearson's Student-like surfaces: $\quad[c] 15: 234,[c] 18$ : 229, [c]22:13\%.

\subsection{2 .}

$\mathrm{D}(\mathrm{x}, \mathrm{y})=\mathrm{x}+\mathrm{y}, \mathrm{D}(\mathrm{x}+\mathrm{y}): \quad[8] 94$.

9.23 .

Normal-negative binomial: · $\cdot[k]$ 13:289

\subsection{4 .}

Edgeworth surface: $\quad[c] 38: 220,[c] 17: 314$.

9.25 .

Rayleigh bivariate: Electrical Engineering, November 1954, p. 1004.

9.26 .

Discussion of "possible" bivariate distributions, Narumi's system; $[c] 15: 7 \%, 209,222$.

Generalization: $\quad[c] 22: 109$.

\subsection{Von Mises-Fisher Distribution}

Generalization of No. 8.17: Proc. Roy. Soc. Lond. Ser. A 217:295, [c]43:344.

9.28

Beta bivariate: $\quad[v] 2: 261$.

9.29 .

$\mathrm{D}(\mathrm{x}, \mathrm{y})=\mathrm{k} \frac{\mathrm{e}^{-\frac{1}{2}\left(\mathrm{ax}^{2}+2 \mathrm{bxy}+\mathrm{cy}^{2}\right)}}{\mathrm{m}^{2}+\left(\mathrm{ax}^{2}+2 \mathrm{bxy}+\mathrm{cy}^{2}\right)}: \quad[v] 3: 153$.

9.30 .

$\mathrm{D}(\mathrm{x}, \mathrm{y})=\mathrm{k}\left[1-\mathrm{a}^{2} \mathrm{x}^{2}-\mathrm{b}^{2} \mathrm{y}^{2}+2 \mathrm{abrxy}\right]^{\mathrm{n}}, \mathrm{n}+1>0, \mathrm{r}^{2}<1$ : $[v] 3: 273$.

9.31. $\mathrm{D}(\mathrm{x}, \mathrm{y})=\mathrm{k} \exp [-\mathrm{Q}(\mathrm{x}, \mathrm{y})] \cdot\left[\mathrm{h}^{2}+\mathrm{Q}(\mathrm{x}, \mathrm{y})\right]^{\mathrm{n}}: \quad[v] 3: 273$,
$[v] 5: 323$.
Defined over $(0,0)(0,1) \quad(1,0)$ from urn model: [v] $3: 328$.

\subsection{3.}

"Correlation by common factor" surface: [c]24: 288.

9.34 .

Johnson's system; ten surfaces obtained by translation: $\quad[c] 36: 297$.

9.35 .

Nine surfaces with Pearson or Bessel marginal distributions: $\quad$ MR5:126

9.36

$\mathrm{D}(\mathrm{x}, \mathrm{y})=\mathrm{C}[(\mathrm{x}-1) !(\mathrm{h}-\mathrm{x}) !(\mathrm{y}-1) !(\mathrm{k}-\mathrm{y}) !]^{-1}: \quad$ Hoel, Intro. to Math. Stat. 180.

9.37.

Type III bivariate, with discussion and calculation of $\mathrm{D}(\mathrm{r}): \quad[e]^{\mathrm{r}} \mathrm{y}: 159$.

9.38 .

$\mathrm{D}(\mathrm{x}, \mathrm{y})=\frac{1}{4}(1+\mathrm{kxy}),|\mathrm{k}| \leq 1,-1 \leq \mathrm{x}, \mathrm{y} \leq 1: \quad[w] 8: 234$.

\section{Miscellaneous Multivariate}

10.1. Wishart Trivariate

$\mathrm{D}(\mathrm{x}, \mathrm{y}, \mathrm{z})=\frac{\mathrm{n}^{\mathrm{n}-1}\left(\mathrm{x} y-\mathrm{z}^{2}\right)^{\frac{1}{2}(\mathrm{n}-4)}}{4 \pi \Gamma(\mathrm{n}-2) \mathrm{M}^{\frac{1}{2}(\mathrm{n}-1)}} \exp \left(-\frac{\mathrm{n}}{2 \mathrm{M}}\left(\mathrm{v}_{2} \mathrm{x}-2 \mu \mathrm{z}\right.\right.$ $\left.\left.+\mathrm{v}_{1} \mathrm{y}\right)\right)$ $\mu=\rho \sigma_{1} \sigma_{2}, \mathrm{M}=\mathrm{V}_{1} \mathrm{~V}_{2}\left(1-\rho^{2}\right): \quad$ [1]397, [3]330, [4]226.

$\operatorname{Ch}(\mathrm{x}, \mathrm{y}, \mathrm{z})=\left(\frac{\mathrm{A}}{\mathrm{A}^{*}}\right)^{\frac{1}{2} \mathrm{n}-1} \quad$ where $\mathrm{A}=\left|\begin{array}{cc}\frac{\mathrm{nv}}{2 \mathrm{M}} & \frac{\mu \mathrm{n}}{2 \mathrm{M}} \\ -\frac{\mu \mathrm{n}}{2 \mathrm{M}} & \frac{\mathrm{v}_{1} \mathrm{n}}{2 \mathrm{M}}\end{array}\right|$ and

$\mathrm{A}^{*}=\left|\begin{array}{cc}\frac{\mathrm{nv_{2 }}}{2 \mathrm{M}}-\text { is } & \frac{\mu \mathrm{n}}{2 \mathrm{M}}-\mathrm{it} \\ -\frac{\mu \mathrm{n}}{2 \mathrm{M}}-\text { it } & \frac{\mathrm{v}_{1} \mathrm{n}}{2 \mathrm{M}}-\mathrm{iu}\end{array}\right|$.

Moments and cumulants: [3]334.

As distribution of normal bivariate variance-covariance: $\quad[c] 10: 510,[c] 21: 164,[c] 27: 230$.

See also: $\quad[k] 9: 243,[u]$ 44:295, J. Soc. Stat. Paris 96:262, [u]29:264, [a]92:580. 
10.2. Wishart Multivariate

$\mathrm{D}\left(\mathrm{x}_{\mathrm{ij}}\right)=\mathrm{K}_{\mathrm{kn}} \mathrm{A}^{(\mathrm{n}-1)} \mathrm{X}^{(\mathrm{n}-\mathrm{k}-2)} \exp \left(-\Sigma \mathrm{a}_{1 \mathrm{j}} \mathrm{x}_{\mathrm{ij}}\right)$,

where $\mathrm{X}=\left|\mathrm{x}_{1 \mathrm{j}}\right|, \mathrm{A}=\left|\mathrm{a}_{\mathrm{ij}}\right|$ and

$\mathrm{K}_{\mathrm{kn}}=\pi^{\frac{1}{\mathrm{k}} \mathrm{k}(\mathrm{k}-1)}\left[\Gamma\left(\frac{1}{2}(\mathrm{n}-1)\right) \ldots \Gamma\left(\frac{1}{2}(\mathrm{n}-\mathrm{k})\right)\right]^{-1}$

$\mathrm{Ch}\left(\mathrm{x}_{1 \mathrm{j}}\right)=\left(\frac{\mathrm{A}}{\mathrm{A}^{*}}\right)^{\frac{1}{2}(\mathrm{n}-1)}$,

where $\quad A^{*}=\left|a_{1 j}-i \epsilon_{1 j} t_{1 j}\right|$ and $\epsilon_{1 j}=\left\{\begin{array}{l}1, i=j \\ \frac{1}{2}, i \neq j\end{array}\right.$

[4]226, [3]331, [1] 391-4, [14]66, [i]30:151, [u]29:260, 271.

Reproductive property: [4]232.

Various properties: [c]20:32, [i]24:185.

Non-central Wishart: Proc. Roy. Soc. Lond. Ser. A. $229: 364$.

See also: $[d] 3: 19 \%,[d] 15: 345,[d] 17: 409,[d] 19$ : 262, [e]3:25, [a]97:120, $[c] 24: 476,[c] 36: 59,[k] c: 244$, [c]38:470, [i] $36: 17,[u] 35: 336$, MR10:387, [b]17:79.

\subsection{Multinomial}

$\mathrm{D}\left(\mathrm{x}_{1}, \ldots, \mathrm{x}_{\mathrm{K}}\right)=\left[\mathrm{n} ! / \Pi\left(\mathrm{x}_{1} !\right)\right] \Pi\left(\mathrm{p}_{1} \mathrm{x}_{1}\right):$ [7]124, [18]1-160.

$[6] 58,[2] 290$

$\operatorname{MGF}\left(\mathrm{x}_{1}, \ldots, \mathrm{x}_{\mathrm{k}}\right)=\left(\mathrm{p}_{\mathrm{k}} \mathrm{e}^{\mathrm{t}_{1}}+\ldots+\mathrm{p}_{\mathrm{k}} \mathrm{e}_{\mathrm{k}}\right)^{\mathrm{n}}: \quad$ [4] 51 .

$\mathrm{E}\left(\mathrm{x}_{\mathrm{i}}\right)=\mathrm{np} \mathrm{p}_{\mathrm{i}}, \operatorname{Var}\left(\mathrm{x}_{\mathrm{i}}\right)=\mathrm{np}_{\mathrm{i}}\left(1-\mathrm{p}_{\mathrm{i}}\right): \quad$ [4]52, [14].35.

Moments: Bull. Amer. Math. Soc. 41:857

Introductory article with applications: [15]36.

PGF : $\quad[18] 1-146$.

Chi-square test: [e]13:2, [c]36:118, [15]739.

Information and estimation: [e]8:325.

MLE: [e]18:139.

Distinguishing between two multinomials, asymptotic form: $[e] \mathrm{y}: 401$.

Trivariate: $\quad[7] 146,[d] 21: 420$.

Bivariate multinomial: [d]23:547, Rev. da Fac. de Ciencias de Lisboa 2 Serie (A) 2:197.

See also: $\quad[d] 8: 127,[d] 21: 416,[e] 11: 367,[d] 25: 772$, [d]28:861, [f]13:451, [t]2:84, Am. Math. Monthly 58: 59, Koninkl. Nederl. Akad. (A) 60:121, Z8:122, MR17:56, MR16:839, MR13:665.

10.4 Tyрэ X Multivariate

$\mathrm{D}\left(\mathrm{x}_{1}, \ldots, \mathrm{x}_{\mathrm{n}}\right)=\mathrm{C} \mathrm{e}^{-\mathrm{x} / \mathrm{b}}$ where $\mathrm{x}=\Sigma \mathrm{x}_{\mathrm{i}}^{2}: \quad[c] 41: 54$. 10.5.

Gamma multivariate: $[e] 11: 45$.

10.6. Student Multivariate

$\mathrm{D}\left(\mathrm{x}_{1}, \ldots, \mathrm{x}_{\mathrm{p}}\right)=\frac{\mathrm{A}^{\frac{1}{2}} \Gamma\left[\frac{1}{2}(\mathrm{n}+\mathrm{p})\right]}{(\mathrm{n} \pi)^{\frac{1}{2} \mathrm{p}} \Gamma\left(\frac{1}{2} \mathrm{n}\right)}\left[1+\frac{1}{\mathrm{n}} \Sigma \mathrm{a}_{\mathrm{ij}} \mathrm{x}_{1} \mathrm{x}_{\mathrm{j}}\right]^{-\frac{1}{2}(\mathrm{n}+\mathrm{p})}$,
Student for $\mathrm{p}=1: \quad[c]_{4} 1: 153$, MR16:602.

See also: $\quad[w] 9: 143$.

10.7. Cauchy Multivariate

$\mathrm{D}\left(\mathrm{x}_{1}, \ldots ., \mathrm{x}_{\mathrm{n}}\right)=\mathrm{C}\left(\mathrm{a}^{2}+\mathrm{x}^{2}\right)^{-\frac{1}{2} \mathrm{~s}-\frac{1}{2}}$, where $\mathrm{x}^{2}=\Sigma \mathrm{x}_{i}^{2}$ : $[c] 41: 54, M R 16: 51$.

$\mathrm{D}\left(\mathrm{x}_{1}, \ldots, \mathrm{x}_{\mathrm{n}}\right)=\mathrm{C}_{\mathrm{n}}\left(1+\sum_{\mathrm{j}=1}^{\mathrm{n}} \mathrm{x}_{y}^{2}\right)^{-\frac{1}{2}(n+1)}: \quad[w] 8: 235$

10.8. Spherical

$\mathrm{D}\left(\mathrm{x}_{1}, \ldots, \mathrm{x}_{\mathrm{n}}\right)=$

$$
(2 \pi)^{-\frac{1}{2} \mathrm{n}} \mathrm{r}^{-\frac{1}{2} \mathrm{n}+1} \int_{0}^{\infty} \rho^{\frac{1}{2} \mathrm{n}} J_{\frac{1}{2} \mathrm{n}-1}(\mathrm{r} \rho) \operatorname{Ch}(\rho) \mathrm{d} \rho,
$$

where $\mathrm{r}=\sqrt{\Sigma \mathrm{x}_{1}^{2}}, \quad \rho=\sqrt{\Sigma \mathrm{t}_{1}^{2}}: \quad[c] 41: 45$.

\subsection{Poisson Multivariate}

Derivation: $\quad[e] 11: 120,[d] 28: 466,[i] 3 \%: 1$.

Without correlation, multiple Poisson

$\mathrm{D}\left(\mathrm{x}_{1}, \ldots, \mathrm{x}_{\mathrm{n}}\right)=$

$$
\begin{aligned}
\exp -\left(\mathrm{k}_{\mathrm{k}}+\ldots+\mathrm{k}_{\mathrm{r}}\right) \frac{\mathrm{k}_{1}^{\mathrm{x}_{1}} \cdot \ldots \cdot \mathrm{k}_{\mathrm{n}}^{\mathrm{x}} \mathrm{.}}{\mathrm{x}_{1} ! \cdot \ldots \mathrm{x}_{\mathrm{n}} !} \\
\quad[\%] 12 \%,[\mathrm{e}] 19: 210,212: 113,410 .
\end{aligned}
$$

10.10. Binomial Multivariate

$\operatorname{MGF}\left(\mathrm{x}_{1}, \ldots, \mathrm{x}_{\mathrm{n}}\right)=\left(1+\Sigma \mathrm{p}_{\mathrm{i}} \mathrm{t}_{\mathrm{i}}+\Sigma \mathrm{p}_{\mathrm{ij}} \mathrm{t}_{\mathrm{i}} \mathrm{t}_{\mathrm{j}}+\ldots\right)^{\mathrm{N}}$ : [e] 11:119, 212:113, 410 .

$\mathrm{D}(\mathrm{x})$, etc., in special case: $\quad[i] 18: 271$.

10.11 .

Negative binomial multivariate: $[i] 18: 274,[i] 19$ : 211, Konikl. Nederl. Akad. (A)60:121.

10.12.

Multinomial multivariate: $[c] 36: 4 \%$.

10.13.

Hotelling multivariate: $[k] 9: 258,[x]$ \%:70.

10.14.

Multivariate distributions obtained from the normal multivariate: $\quad[i] 27: 235,[i] 28: 20$.

10.15 .

Generalization of No. 9.14: [k]9:245.

10.16 .

Generalization of No. 8.3: [k]11:136. 
10.17.

Generalization of No. 8.6, No. 9.29: $\quad[v] 3: 153$.

10.18

Hypergeometric multivarıate: [e]15:391, [f]13: 488, MR17:634, MR12:722.

10.19.

Gram-Charlier multivariate: $\quad M R 14: 486$.

10.20. Run Length

$\mathrm{D}(\mathrm{x}): \quad[d] 11: 367,[4] 202,206,[s] 5: 143$.

\subsection{Beta Multivariate}

Tolerance limits: [4]94.

\section{References}

\section{Journals}

[a] Journal of the Royal Statistical Society, Series A

[b] Journal of the Royal Statistical Society, Series B

[c] Biometrika

[d] Annals of Mathematical Statistics

[e] Sankhyā

[f] Biometrics

[g] Journal of the American Statistical Association

[h] Nordisk Statistisk Tidskrift

[h'] Nordic Statistical Journal

[i] Skandinavisk Aktuarietidskrift

[j] Bell System Technical Journal

[k] Annals of Eugenics, Annals of Human Genetics

[1] Econometrica

[m] Applied Statistics

[n] Metron

[o] Annals of the Institute of Statistical Mathematics

[p] Journal of the Institute of Actuaries Students' Society

[q] Bulletin of Mathematical Statistics

[r] Reports of Statistical Application Research, Union of Japanese Scientists and Engineers

[s] Statistica (Neerlandica)

[t] Calcutta Statistical Association Bulletin

[u] Proceedings of the Cambridge Philosophical Society

[v] Trabajos de Estadistica

[w] Mitteilungsblatt fur Mathematische Statistik

[x] Journal of the Indian Society of Agricultural Statistics

[y] Revue de l'Institut International de Statistique

\section{Books}

[1] Cramér, H., Mathematical methods of statistics (Princeton Univ. Press, Princeton, N.J., 1946).

[2] Kendall, M. G., The advanced theory of statistics, vol. 1 (Charles Griffin \& Co., London, England, 1943).

[3] Kendall, M. G., The advanced theory of statistics, vol. 2 (Charles Griffin \& Co., London, England, 1946).

[4] Wilks, S. S., Mathematical statistics (Princeton Univ. Press, Princeton, N.J., 1947).

[5] Arley, N. and Buch, K. R., Introduction to the theory of probability and statistics (John Wiley \& Sons, New York, N.Y., 1950).

[6] Mood, A. M., Introduction to the theory of statistics (McGraw-Hill Book Co., Inc., New York, N.Y., 1950).

[7] Feller, W., An introduction to probability theory and its applications (John Wiley \& Sons, New York, N.Y., 1951).

[8] Munroe, M. E., Theory of probability (McGraw-Hill Book Co., Inc., New York, N.Y., 1951).
[9] Uspensky, J. V., Introduction to mathematical probability (McGraw-Hill Book Co., Inc., New York, N.Y., 1937).

[10] Weatherburn, C. E., A first course in mathematical statistics (Cambridge Univ. Press, Cambridge, England, 1946).

[11] Elderton, W. P., Frequency curves and correlation (Charles \& Edwin Layton, London, England, c. 1906).

[12] Bell, D. A., Statistical methods in electrical engineering (Chapman and Hall, London, England, 1953).

[13] Neyman, J., (editor) Proceedings of the Berkeley Symposium on Mathematical Statistics and Probability (Univ. of Calif. Press, Berkeley, Calif., 1945).

[14] Rao, C. R., Advanced statistical methods in biometric research (John Wiley \& Sons, New York, N.Y., 1952).

[15] Hald, A., Statistical theory with engineering applications (John Wiley \& Sons, New York, N.Y., 1952).

[16] Neyman, J., Proceedings of the Second Berkeley Symposium on Mathematical Statistics and Probability (Univ. of Calif. Press, Berkeley, Calif., 1951).

[17] Haller, B. Verteilungsfunktionen und ihre Auszeichnung durch Funktionalgleichungen, Mitteilungen der Vereinigung schweizerischer Versicherungsmathematiker; 45 Band, Heft 1, (April 1945), pp. 97-163. Translated by R. E. Kalaba, and published by the RAND Corp. under the title, A Summary of Known Distribution Functions, T-27, (Jan. 1953).

[18] Eisenhart, C., and Zelen, M., Elements of probability, ch. 12, Handbook of Physics (McGraw-Hill Book Co., Inc., New York, N.Y., 1958).

\section{Index to Distributions}

Arfwedson distribution

Bayes' distribution

Bayes' theorem -

Behren's problem
Bernoulli distribution

Bessel distribution

Beta distribution

Beta bivariate distribution

Beta multivariate distribution

Beta of the first kind distribution.

Beta of the second kind distribution

Bhattacharyya bounds

Bionomial distribution

Binomials added distribution

26, 32 , No. $3.1,37$, No. $3.2,38,40,41,42,43$

Binomial multivariate distribution

Bipolar distribution
Borel-Tanner distribution

Borel-Tanner distribution
Bose distribution
Bravais distribution

Cauchy distribution $29,30,45$, No. $7.4,48$, No. $7.5,48$, No. $7.6,49$, No. $7.7,49,53$ Cauchy bivariate distribution

Cauchy median distribution

Cauchy multivariate distribution

Causal distribution.

Chi-square distribution.--.-.
Cigarette card distribution

No. $9.1,55$

No. $8.13,51$

No. $3.7,39$

Circular normal distribution

No. 8.15, 51

Compound normal distribution

Compound Poisson distribution

Contagious distribution

Contagious bivariate distribution

Continuous Lexian distribution.

Correlation distribution

Correlation determinant distribution

Correlation ratio distribution.

D distribution

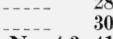

No. $4.6,42$

Deterministic distribution

Discrete Lexian distribution

Discrete lognormal distribution

Discrete normal distribution.

Diserete rectangular distribution

Discrete rectangular distribution

Discrete type III distribution

Double exponential distribution

Double Pareto distribution.

Double Pareto distribution.

No. $8.6,50$

No. $5,14,46$ No. $5.9,44$

Edgeworth surface

Elfving distribution

Erlang distribution

Eulerian distribution

Exceedance distribution

Exponential distribution

Extreme value distribution.

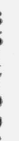
29
46

年


F distribution

Fermi-Dirac distribution

Fifteen-constant surface.

Filon-Isserlis surface

Fisher

Fisher distribution

Fisher's F distribution

Fisher's logarithmic series distribution

Furry distribution

Galton-Macalister distribution

Gamma distribution

Gamma bivariate distribution

Gamma-normal distribution

Garwood distribution

Generalized binomial distribution

Generalized normal distribution

Generalized Pareto distribution

Generalized Poisson distribution

Generalized Student distribution

Generalized type III distr

Geometric distribution

Ghosh distribution

Gibrat distribution

Gram-Charlier multivariate distribution

Gumbel distribution

Halphen distribution

Hansmann distribution

Helmert distribution

Hotelling

Hyperbolic error distribution

Hypergeometric bivariate distribution

Hotelling multivariate distribution

Hypergeometric distribution

IIypergeometric multivariate distribution

Inverse Gaussian distribution

Inverse hypergeometric

Inverted beta distribution

Irwin-Hall distribution

Ising-Stevens distribution

(n)

Johnson's system.

Kapetyn distribution

Kendall distribution.

Koopman distribution

Koopman-Darmois distribution

Kullback distribution.

32,33, No. $6.4,47,50$

No. 9.9, 55

27,40, No. $4.5,41,50,53$

Laplace distribution

Laplace-Gauss distribution

Legendre functions

Leipnik distribution

Lexian distribution.

Logarithmic non-central Chi-square distribution

Logistic distribution

Lognormal distribution

Lognormal bivariate distribution

Log Pearson distribution.

4 , No. $6.7,47$

No. $4.11,42$

45,46

Mahalanobis distribution

Matching distribution

Maxwell-Boltzmann distribution

Mellin transformation

Mill's ratio

Miller distribution

Multinomial distribution

Multinomial bivariate distribution

Multinomial multivariate distribution

Multinomial trivariate distribution

Multiple correlation distribution

Multiple Poisson distribution

No. $8.75,54$

56

No. $1.6,30$

No. $8.16,5$
, No.

No. $8.4,50$

$29,30,45$, No. $7.8,49,53$

$\begin{array}{rr} & 30 \\ \text { No. 5.1, } & 37 \\ & 38 \\ 36\end{array}$

24 , No. $1.8,30,51$

$\mathbf{5 1}$

55

Narumi's system

Negative binomial distribution

Negative binomial bivariate

Negative binomial multivariate distribution

Negative exponential distribution

Negative hypergeometric distribution

Neyman type A distribution

Neyman-Pearson theory

Noncentral Chi-square distribution

Noncentral Fisher distribution.

Noncentral Helmert distribution

Noncentral Snedecor distribution

Noncentral Student distribution

Noncentral Wishart distribution

Nonnull $\mathrm{t}^{2}$ distribution

Normal distribution

26, No. 1.2, 29, No. 1.3, 29, No. 1.4, 29, 26-30, 32, 35, 40, 42, 43, 48, 49, 53

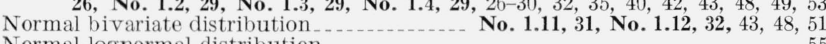

Normal-lognormal distribution

No. $1.11,31$, No. $1.12,32,43,48,51$

Normal-negative binomial distribution

Normal regression slope distribution

Normals added distribution

No. $7.3,48$

No. $7.3,48$

Normal trivariate distribution.

No. $1.13,32-33,43$

Parabolic distribution

No. $5.6,44$
Pareto distribution.

Partial correlation distribution

Pascal distribution.

Pearson's differential equation

Pearson distribution

Pearson's Student-like surfaces

Perks distribution

Pitman.

Planck's radiation function

Poisson distribution

Poisson bivariate distribution

Poisson-Lexian distribution

Poisson multivariate distribution

Poisson's first law of error

Pollaczek-Geiringer distribution

Polya-Aeppli distribution

Polya-Eggenburger distribution
Polya distribution

Rank variate distribution

Rayleigh distribution

Rayleigh bivariate distribution

Reciprocal type III distribution

Reciprocal truncated binomial distribution

Rectangular distribution

Rectangular geometric mean distribution

Rectangular mean distribution

Rectangulars added distribution

Rhodes surface

Right triangular distribution

Romanovsky distribution
Run length distribution

Rutherford contagious distribution

No. $6.5,47$

No. $5.5,44$
No. $3.5,39$

$26,29,36,40,43,46,50,53$ 24, No. 8.1, 50, 56

$24,34,35,39$, No. 4.1, 39, 42,43

No. $9.3,55$

No. 10.9, 57

No. $4.7,42$

No. $8.90,54$

39,42

No. $4.9,42$

No. $8.9,51$

33, No. $2.8,36$

\begin{tabular}{l}
36 \\
56 \\
36 \\
39 \\
\hline 5
\end{tabular}

No. $5.13,45$
No. $8.12,51$

No. 5.16, 46

Ne. 46

$\begin{array}{r}44 \\ \text { No. } 10.20,58 \\ \hline\end{array}$

Seminormal distribution.

Semitriangular distribution

Serial correlation distribution

Sheppard

Snedecor distribution

Snedecor test

Spearman

Spearman's rank correlation distribution

No.

Spherical distribution

Stevens-Craig distribution

Stevens-Fisher distribution

Stirlings number

Student bivariate distribution

Student-Fisher theorem

Student multivariate distribution

Student's hypothesis

Student test

36
44
No. 5.1, 42

30,45

$\mathrm{t}$ distribution

Thompson distribution

Tine distribution

Triangular distribution

Triangular bivariate distribution

Truncated binomial distribution

Truncated exponential distribution

Truncated lognormal distribution

Truncated normal distribution
Truncated Poisson distribution

Truncated type III distribution

Type harmonique distribution

Type I distribution

Type II distribution

Type II distribution 29 , No. 5.4, 43, 44, 48, 51
Type III distribution
29, No. $2.1,33$, No. 2.2, 34, No. $2.3,35$, No. $2.4,35,39,41,43,50,55$

Type III bivariate distribution

Type III discrete distribution

Type III trivariate distribution

Type IV distribution

Type V distribution.

Type VI distribution.

Type VII distribution.

Type VIII distribution

Type IX distribution
Type $\mathrm{X}$ distribution

Type X distribution $\mathrm{X}$ multivariate distribution

Type X multivariate
Type XI distribution

Type XII distribution

$24,26,27,28,29,32,43,44,46,47$, No. $7.2,48$

No. $9.2,55$

No. 10.6, 57

28
33

Uniform distribution

Uniform bivariate distribution

No. $7.2,48$

No. $5.10,44$

No. $3.3,38$

34,35

No. $1.5,30$

34

Variance ratio distribution.

Von Mises distribution

Von Mises-Fisher distribution

No. $8.17,51$

Weibull distribution

No. $8.34,52$

Wiltaker function

Wishart distribution

Wishart multivariate distribution

Wishart trivariate distribution.

Wrapped-up normal distribution

Wrapped-up Cauchy distribution.

32,33, No. $2.10,37$

No. $10.2,57$

No. 10.1, 56

No. $1.9,31$

Yule distribution.

51

$\mathrm{z}$ distribution. 
V. Appendix. Chronological. Table

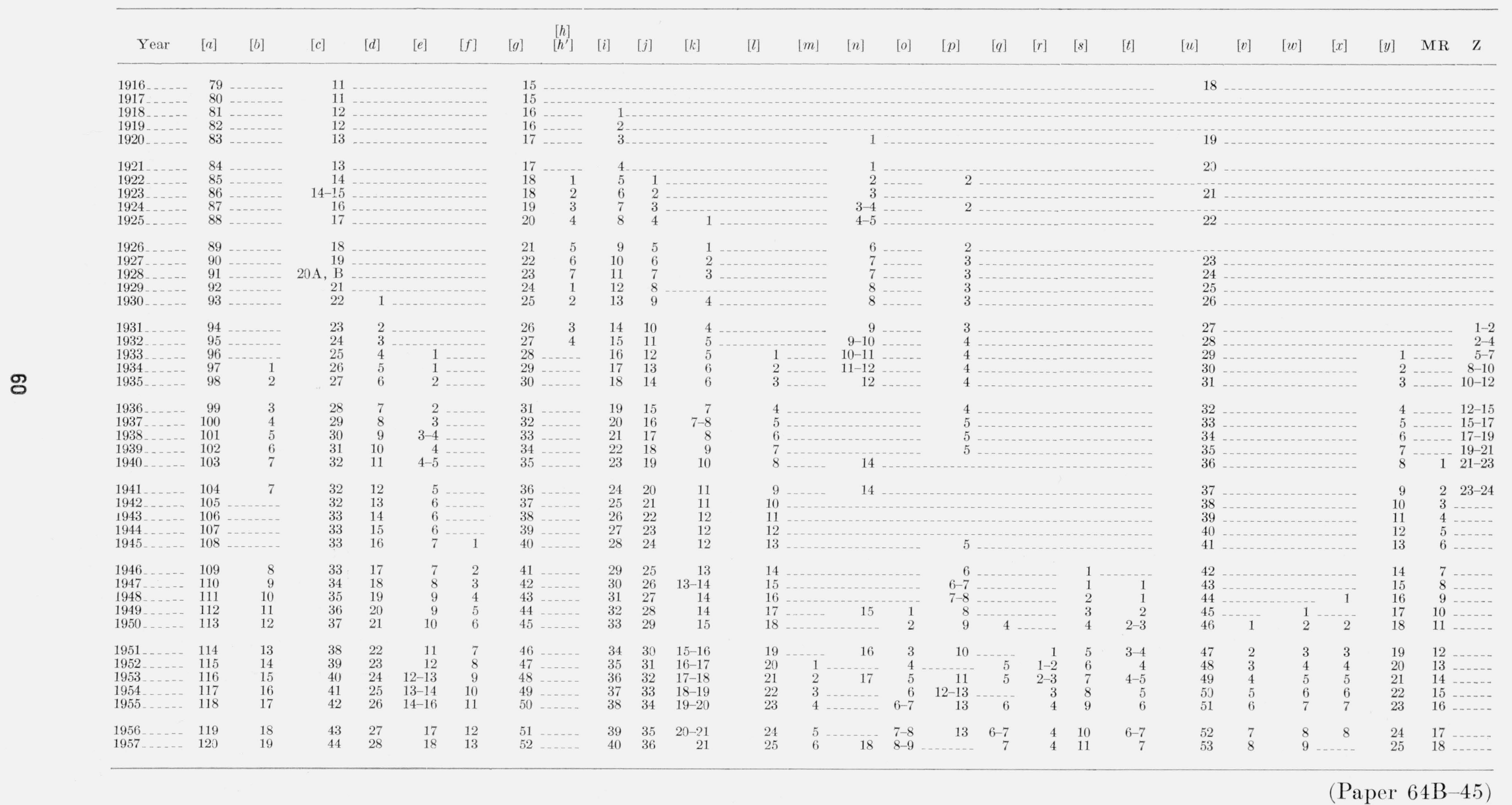

